Florida International University FIU Digital Commons

FIU Electronic Theses and Dissertations

University Graduate School

$11-17-2017$

\title{
An Integrated Multi-modal Registration Technique for Medical Imaging
}

Xue Wang

xwang040@fiu.edu

DOI: $10.25148 /$ etd.FIDC004051

Follow this and additional works at: https://digitalcommons.fiu.edu/etd

Part of the Biomedical Commons, and the Signal Processing Commons

\section{Recommended Citation}

Wang, Xue, "An Integrated Multi-modal Registration Technique for Medical Imaging" (2017). FIU Electronic Theses and Dissertations. 3512 .

https://digitalcommons.fiu.edu/etd/3512

This work is brought to you for free and open access by the University Graduate School at FIU Digital Commons. It has been accepted for inclusion in FIU Electronic Theses and Dissertations by an authorized administrator of FIU Digital Commons. For more information, please contact dcc@fiu.edu. 


\section{FLORIDA INTERNATIONAL UNIVERSITY}

Miami, Florida

\section{AN INTEGRATED MULTIMODAL REGISTRATION TECHNIQUE FOR MEDICAL IMAGING}

A dissertation submitted in partial fulfillment of the requirements for the degree of DOCTOR OF PHILOSOPHY

in

ELECTRICAL ENGINEERING

by

Xue Wang 
To: Dean John Volakis

College of Engineering and Computing

This dissertation, written by Xue Wang, and entitled An Integrated Multimodal Registration Technique for Medical Imaging, having been approved in respect to style and intellectual content, is referred to you for judgment.

We have read this dissertation and recommend that it be approved.

Jean H. Andrian

Armando Barreto

Mercedes Cabrerizo

Naphtali Rishe

Malek Adjouadi, Major Professor

Date of Defense: November 17, 2017

The dissertation of Xue Wang is approved.

Dean John L. Volakis

College of Engineering and Computing

Andrés G. Gil

Vice President for Research and Economic Development and Dean of the University Graduate School

Florida International University, 2017 
C Copyright 2017 by Xue Wang

All rights reserved. 


\section{DEDICATION}

I dedicate this dissertation to my supportive husband, Xianming Qidiao, to my lovely son, Edward R. Ming, for making this work meaningful. I also dedicate this work to my beloved mother, Jirong Yang, and father, Yuehua Wang. With their unwavering support, love and understanding, I found all the strength to carry on with my graduate studies, all the way to the Ph.D. degree. 


\section{ACKNOWLEDGMENTS}

First and foremost, I would like to express my deepest gratitude to my major professor, Dr. Malek Adjouadi, for his excellent guidance, caring, patience, and invaluable insights throughout all the stages of researching and writing this dissertation, as well as for the financial support provided to me by the National Science Foundation through the CATE Center for the completion of this dissertation. He provided unwavering support during my Ph.D., patiently corrected my writing and generously paved the way for my development as a researcher. His passion and dedication are incomparable and always inspire me.

Next, I wish to express my great appreciation to my committee members, Dr. Jean H. Andrian, Dr. Armando Barreto, Dr. Mercedes Cabrerizo and Dr. Naphtali Rishe, for their valuable discussions improving the quality of this dissertation.

Furthermore, I would like to acknowledge the support provided from the Department of Electrical and Computer Engineering at Florida International University, and the critical support provided by the National Science Foundation (NSF) under grants: CNS-1532061, CNS-1551221, CNS-1042341, CNS-1429345, and IIP 1338922. The generous support of the Ware Foundation is also greatly appreciated.

I would also like to extend my gratitude to all my lab mates at the Center for Advanced Technology and Education for creating an amazing working environment, especially, Dr. Jin Wang, Dr. Zhenzhong Wang, and Dr. Lilin Guo, whose encouragement greatly helped me in achieving my research goals; to the ECE department staff, especially Ms. Pat Brammer and Mr. Oscar Silveira, who have given me great help and support. 


\section{ABSTRACT OF THE DISSERTATION}

\section{AN INTEGRATED MULTIMODAL REGISTRATION TECHNIQUE FOR MEDICAL \\ IMAGING}

by

Xue Wang

Florida International University, 2017

Miami, Florida

\section{Professor Malek Adjouadi, Major Professor}

Registration of medical imaging is essential for aligning in time and space different modalities and hence consolidating their strengths for enhanced diagnosis and for the effective planning of treatment or therapeutic interventions. The primary objective of this study is to develop an integrated registration method that is effective for registering both brain and whole-body images. We seek in the proposed method to combine in one setting the excellent registration results that FMRIB Software Library (FSL) produces with brain images and the excellent results of Statistical Parametric Mapping (SPM) when registering whole-body images. To assess attainment of these objectives, the following registration tasks were performed: (1) FDG_CT with FLT_CT images, (2) pre-operation MRI with intra-operation CT images, (3) brain only MRI with corresponding PET images, and (4) MRI T1 with T2, T1 with FLAIR, and T1 with GE images. Then, the results of the proposed method will be compared to those obtained using existing state-of-the-art registration methods such as SPM and FSL. 
Initially, three slices were chosen from the reference image, and the normalized mutual information (NMI) was calculated between each of them for every slice in the moving image. The three pairs with the highest NMI values were chosen. The wavelet decomposition method is applied to minimize the computational requirements. An initial search applying a genetic algorithm is conducted on the three pairs to obtain three sets of registration parameters. The Powell method is applied to reference and moving images to validate the three sets of registration parameters. A linear interpolation method is then used to obtain the registration parameters for all remaining slices. Finally, the aligned registered image with the reference image were displayed to show the different performances of the 3 methods, namely the proposed method, SPM and FSL by gauging the average NMI values obtained in the registration results. Visual observations are also provided in support of these NMI values. For comparative purposes, tests using different multi-modal imaging platforms are performed. 


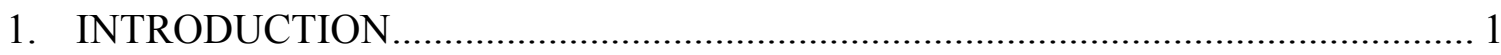

1.1 Retrospective on Registration in Medical Images .............................................

1.2 General Statement of the Research Area ..........................................................11

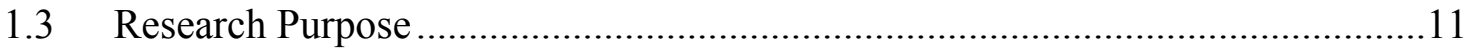

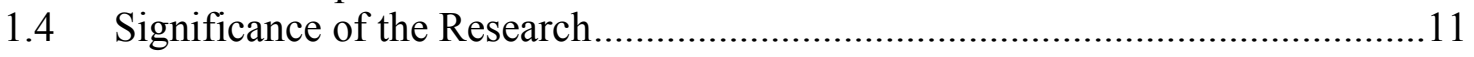

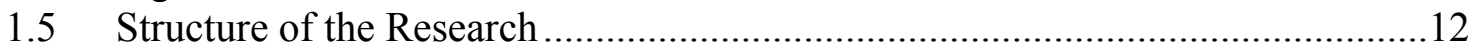

2. A SEMI-AUTOMATIC REGISTRATION METHOD OF COMPUTED

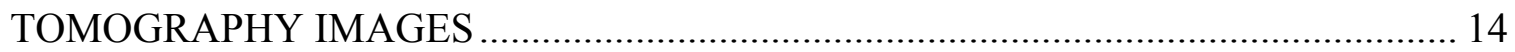

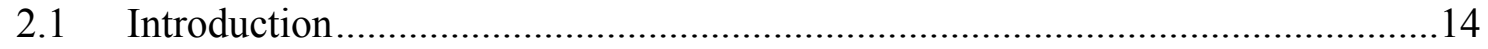

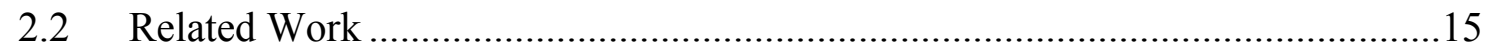

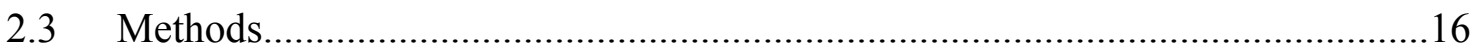

2.3.1 Control Points Selection ..................................................................... 16

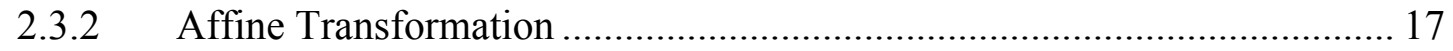

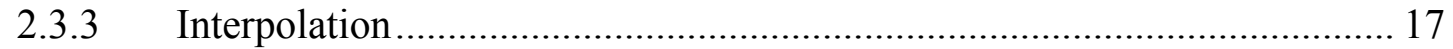

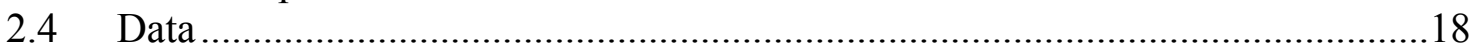

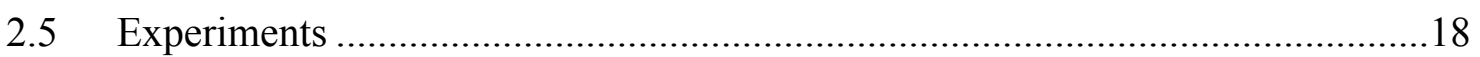

2.5.1 Minimizing Errors in Manual Selection of Control Points ......................... 18

2.5.2 Testing the Middle Slice of FDG_CT from Set 1 to 5 ………………......... 19

2.5.3 Testing All Slices in Set 1 through 5 .................................................. 19

2.5.4 Experimental Procedure....................................................................... 20

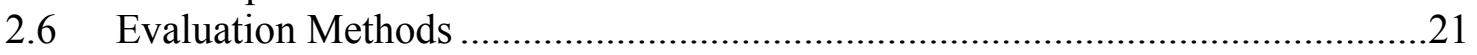

2.6.1 Mutual Information and Normalized Mutual Information.......................... 21

2.6.2 Alignment Metric .................................................................................. 22

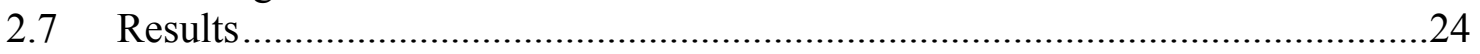

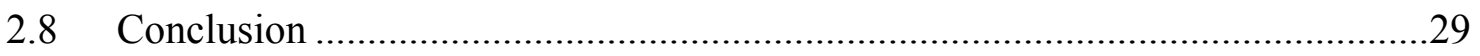

3. AUTOMATIC REGISTRATION OF FDG_CT AND FLT_CT IMAGES ............... 30

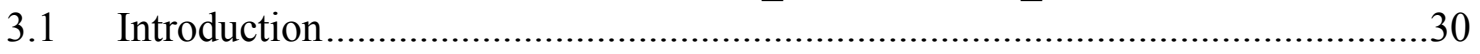

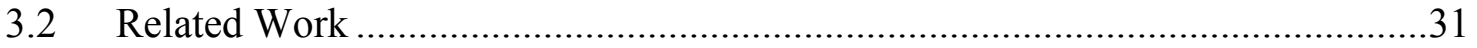

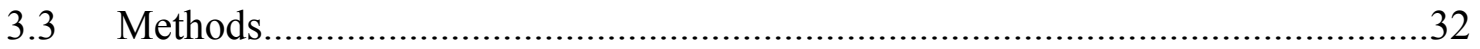

3.3.1 Image Pre-processing Methods .............................................................. 32

3.3.2 The procedures of GPW Method …………………............................. 35

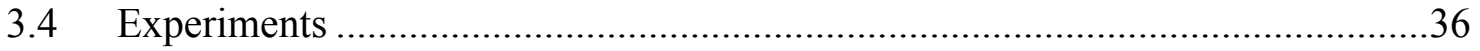

3.4.1 Performance Verification of the Powell Method (PM)................................ 36

3.4.2 Verification of Wavelet-Modified Genetic Algorithm (GA)...................... 39

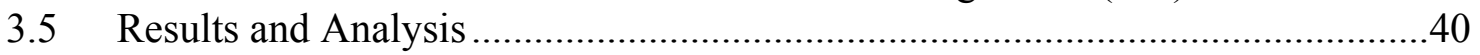

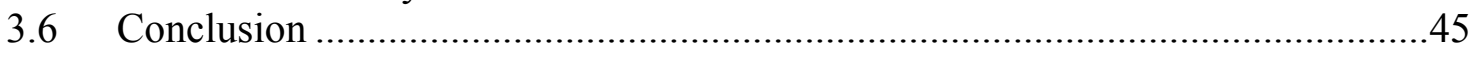

4. MULTI-MODALITY MEDICAL IMAGE REGISTRATION ……………………... 47

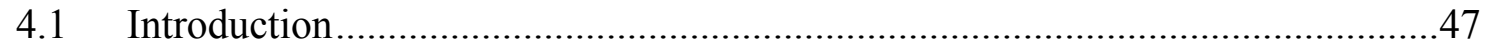

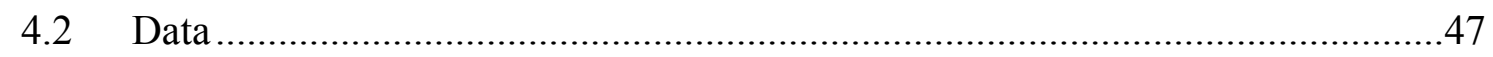

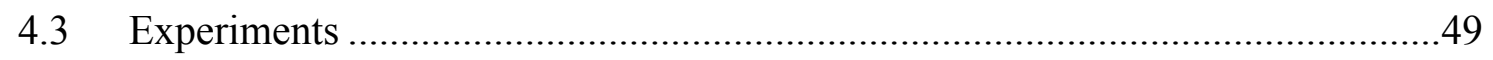


4.3.1 The Goal of the Four Experiments..................................................... 49

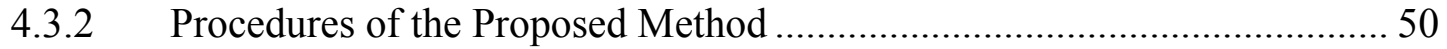

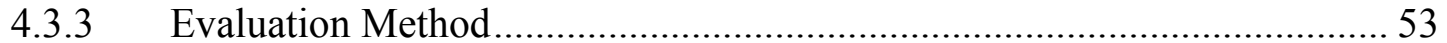

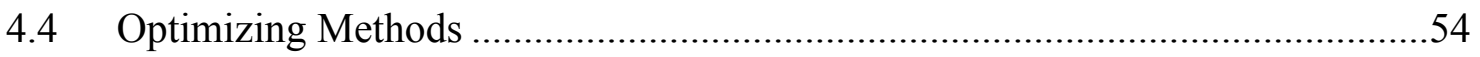

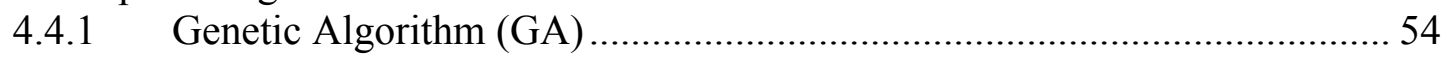

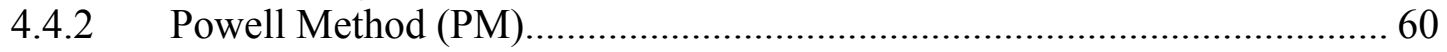

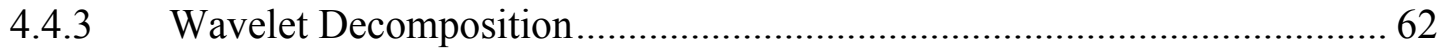

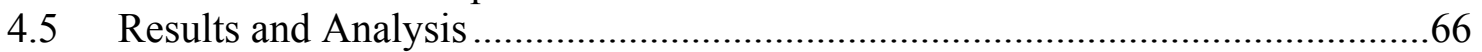

4.5.1 Registration Results of Experiment 1-FDG_CT and FLT_CT .................66

4.5.2 Registration Results of Experiment 2-Pre-MRI and Intra-CT ................ 71

4.5.3 Registration Results of Experiment 3-MRI and PET ............................ 75

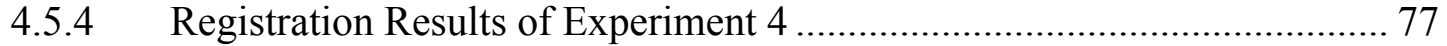

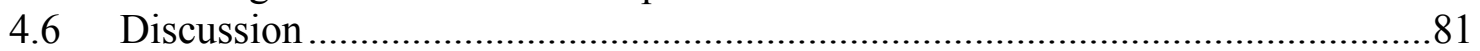

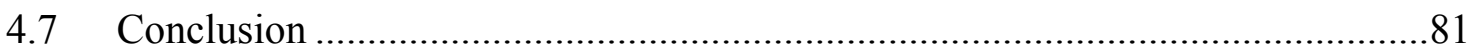

5. CONCLUSIONS AND FUTURE WORK ....................................................... 84

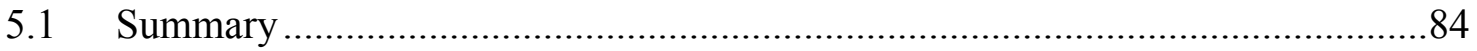

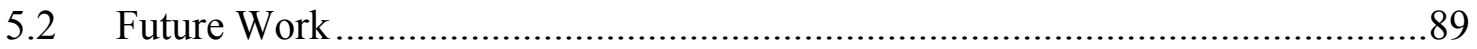

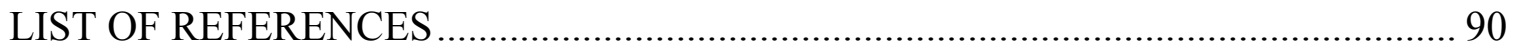

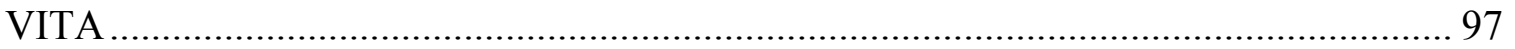




\section{LIST OF TABLES}

TABLE

PAGE

Table 2.1 Evaluation for sets 1 through 5: $\mathrm{I}$ is the input image, $\mathrm{R}$ is the reference image,

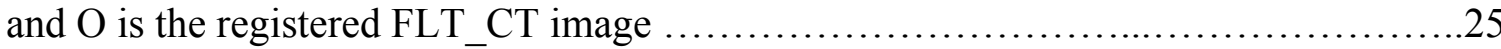

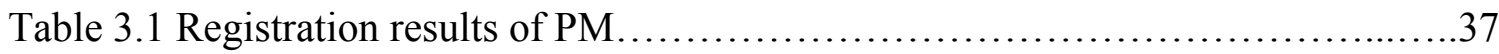

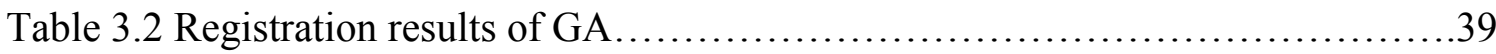

Table 3.3 Register FDG_CT and FLT_CT images by GPW $\ldots \ldots \ldots \ldots \ldots \ldots \ldots \ldots \ldots \ldots . \ldots \ldots$

Table 4.1 Example of Fitness Proportionate Selection............................57

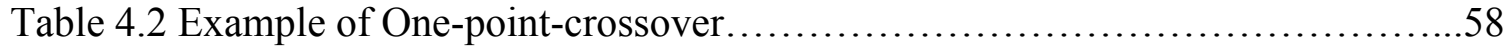

Table 4.3 Processing times of wavelet decomposition, GA and the total...............65

Table 4.4 Processing time and NMI comparison for the 5 data sets of experiment $1 \ldots . . .71$

Table 4.5 Processing time and NMI comparison for the 5 data sets of experiment $2 \ldots . . .75$

Table 4.6 Processing time and NMI comparison for the 5 data sets of experiment $3 \ldots . . .77$

Table 4.7 Processing time and NMI comparison for the 5 data sets of experiment $4 \ldots \ldots .81$ 


\section{LIST OF FIGURES}

FIGURE

PAGE

Fig. 2.1 The registered image with 4 blue "+" marks overlapping the reference image with 4 red "+" marks

Fig. 2.2 Images (a) and (b) are the input image and the reference image with four blue control points marked in each of them. Image (c) is overlapping the green reference image, slice No. 93 of FDG_CT of set 1, and the red input image which is rotating the green image 90-degree counter-clockwise. Image (d) is overlapping the output image and the reference image.

Fig. 2.3 Set 1: 3 slices for the first registration. Images (a) are the first, the middle, and last of the unregistered slices; Images (b) show the registered slices ...................26

Fig. 2.4 The AM for set 1 to 5. Green data 1 is the AM of FLT_CT and FDG_CT; Blue data 2 is the AM of registered image and FDG_CT. Blue data is higher than green data (registration improved) in these parts: (a) from slice No.1 to slice No.11 and from slice No. 105 to slice No.186; (b) from slice No. 40 to slice No. 244; (c) and (d) all of the slices;(e) from slice No. 139 to slice No. 244 in set 5 .

Fig. 2.5 Set 1 to 5: Overlapped the unregistered blue FLT_CT and red FDG_CT; overlapped are the registered green FLT_CT and red FDG_CT. These exemplify typical slice and whole body registration for all 5 patients. .28

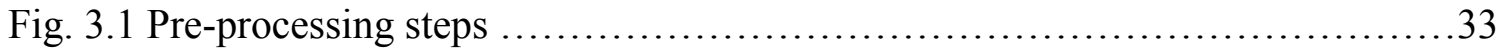

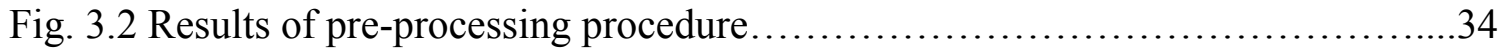

Fig. 3.3 The GPW integrated structure...................................... 35

Fig. 3.4 Registration with GA optimization.................................... 36

Fig. 3.5 Registration with PM optimization..................................... 36

Fig. 3.6 Registration results of experiment \# 1 using PM............................ 38

Fig. 3.7 Registration results of slice No.92 FDG_CT and FLT_CT image..............42

Fig. 3.8 Registration results of slice No.93 FDG_CT and FLT_CT image...............42

Fig. 3.9 Registration results of slice No.94 FDG_CT and FLT_CT image...............43

Fig. 3.10 Registration results of slice No.95 FDG_CT and FLT_CT image.............43 
Fig. 3.11 Registration results of slice No.96 FDG_CT and FLT_CT image.............44

Fig. 3.12 Registration results of slice No.97 FDG_CT and FLT_CT image.............44

Fig. 3.13 Registration results of slice No.98 FDG_CT and FLT_CT image.............45

Fig. 4.1 Flowchart of the proposed method.....................................53

Fig. 4.2 Flowchart of the Genetic Algorithm..................................55

Fig. 4.3 Selection operation................................................57

Fig. 4.4 Flowchart of PM..................................................61

Fig. 4.5 Wavelet Decomposition to the second level...............................62

Fig. 4.6 Original slice \# 93 of FDG_CT image, image size: 512 -by-512.............63

Fig. 4.7 Wavelet decomposition to the first level, image size: 256-by-256, low-frequency component: L1, and high-frequency components: H1, D1, and V1......64

Fig. 4.8 Wavelet decomposition to the second level, image size: 128-by-128, low-frequency component: L2, and high-frequency components: H2, D2, and V2......64

Fig. 4.9 Original images.................................................67

Fig.4.10 Patient \# 1 of data set \#1, (a) Original FDG_CT (appears in red) and FLT_CT (appears in blue) are overlapped; (b) Registration results using SPM12; (c) Registration results using FSL; and (d) Registration results using the proposed method..............69

Fig. 4.11 Enlarged spine in transverse image...................................69

Fig. 4.12 Enlarged skull in sagittal image....................................69

Fig. 4.13 Enlarged spine and rib in sagittal image.................................. 70

Fig. 4.14 Original images................................................... 72

Fig. 4.15 Patient \# 1 of data set \#2, (a) Original pre-operation MRI (appears in red) and intra-operation CT (appears in blue) are overlapped; (b) Registration results using SPM12; (c) Registration results using FSL; and (d) Registration results using the proposed method....................................................... 74

Fig. 4.16 Enlarged skull in the coronal image.................................. 74 
Fig. 4.17 Enlarged skull in the sagittal image.

Fig. 4.18 . Patient \# 1 of data set \# 3, (a) Original MRI (appears in red) and PET (appears in blue) are overlapped; (b) Registration results using FSL ;(c) Registration results using the proposed method

Fig. 4.19 Registration of T1 and T2 (a) the original T1 (appears in red) and T2 (appears in blue) are overlapped; (b) Registration results using SPM12; (c) Registration results using FSL; and (d) Registration results using the proposed method....................78

Fig. 4.20 Registration of T1 and FLAIR (a) the original T1 (appears in red) and FLAIR (appears in blue) are overlapped; (b) Registration results using SPM12; (c) Registration results using FSL; and (d) Registration results using the proposed method..............79

Fig. 4.21 Registration of T1 and GE (a) the original T1 (appears in red) and GE (appears in blue) are overlapped; (b) Registration results using SPM12; (c) Registration results using FSL; and (d) Registration results using the proposed method.... 


\section{ABBREVIATIONS AND ACRONYMS}

$\begin{array}{ll}\text { CT } & \text { Computed Tomography } \\ \text { MRI } & \text { Magnetic Resonance Imaging } \\ \text { SPECT } & \text { Single Photon Emission Computed Tomography } \\ \text { PET } & \text { Positron Emission Tomography } \\ \text { 18F-FDG } & \text { 18F-fluorodeoxyglucose } \\ \text { 18F-FLT } & \text { 18F-fluorothymidine } \\ \text { GA } & \text { Genetic Algorithm } \\ \text { GPW } & \text { Genetic-Powell-Wavelet } \\ \text { PM } & \text { Powell Method } \\ \text { FSL } & \text { FMRIB Software Library } \\ \text { SPM } & \text { Statistical Parametric Mapping } \\ \text { MI } & \text { Mutual Information } \\ \text { NMI } & \text { Normalized Mutual Information } \\ \text { AM } & \text { Alignment Metric } \\ \text { FLAIR } & \text { Fluid-Attenuated Inversion Recovery Image } \\ \text { GE } & \text { Gradient and Spin-Echo Images }\end{array}$




\section{INTRODUCTION}

\subsection{Retrospective on Registration in Medical Images}

The difficulties medical experts are facing extend beyond the need for the fusion of different imaging modalities, which in itself is a challenging problem, in order to provide better assessments of the needed treatment [1, 2]. Such a fusion becomes more meaningful only if it is backed by an effective and accurate registration process that consolidates the strengths that each of the modalities brings forth. The contentious issues involved with registration is due to the fact that not all modalities are aligned onto a same coordinate system, and the transformations that led to this misalignment are often not known. Of course, one way to overcome this challenge is to align in time and space by having simultaneous recordings performed using different modalities. Such are the cases of (1) EEG-triggered fMRI, where the source localization of epilepsy onset is validated through the BOLD effect in the fMRI, and where the EEG recording is done while a patient is inside the MR machine, (2) PET scans performed on hybrid machines that

combine PET or SPECT with CT scanners (PET/CT or SPECT/CT) in order to consolidate anatomical with metabolic activity or function of a specific region of interest under a given pathology.

Registration of medical images could also involve a single imaging modality but with two different radiotracers as in the widely used 18F-fluorodeoxyglucose (18F-FDG) radiotracer, which serves as a good imaging tool in Computed Tomography (CT) and is 
essential for cancer diagnosis as FDG uptake is higher in cancerous lesions and lower in benign ones; while $18 \mathrm{~F}$-fluorothymidine (18F-FLT) uptake is shown to be closely correlated with cellular proliferation. The registration of both of these tracers, FDG and FLT, complement one another to increase both sensitivity and specificity for imaging cancer. Accurate registration of both of these imaging modalities is thus sought in this study, as an example, to optimize the results of the diagnosis. This paper describes a novel feature-based registration method, which employs affine transformation and linear interpolation for FDG_CT and FLT_CT image modalities. Instead of using one set of affine transformation parameters, three slices have been selected to calculate all of the transformation parameters for CT image registration by linear interpolation. Thus, effective registration of these two CT images could prove very useful for diagnosis, including improved means for quantization and visualization.

As another example of multimodal imaging registration is in integrating the use of MR and CT, as the former is better suited for delineation of tumor tissue (and has in general better soft tissue contrast), while the latter is needed for accurate computation of the radiation dose. Another eminent example is in the area of epilepsy surgery. Patients may undergo various MR, CT, and DSA studies for anatomical reference; ictal and interictal SPECT studies; MEG and scalp and/or intra-cranial (subdural or depth) EEG, as well as FDG and/or C-Flumazenil PET studies. Registration of the images from practically any combination will benefit medical experts in surgical planning. 
In Alzheimer's disease (AD) research, the interaction of amyloid load through PET imaging, and regional cortical thickness through MRI are also extensively studied; with some adding cognition and APOE genotype for a more thorough assessment by combining neuroimaging with neuropsychological testing and the APOE gene.

There are many different kinds of medical images that have both advantages and disadvantages, for example, computed tomography (CT) images, contrast CT images, magnetic resonance images (MRI), weighted MRI, positron emission tomography (PET), and so forth. Hence registering these modalities will consolidate their strengths while overcoming their singular limitations. CT is especially useful for soft tissue, such as the brain, the thoracic and abdominal viscera, providing precise details for the physician. However, CT scanning will expose patients to a dose of radiation many times higher than that of X-rays. MRI also provides precise imaging, especially for delineating tumors and for extracting key morphological measures and features. MRI does not expose patients to radiation, but certain patients do not tolerate the confinements of the scanner bore of MR machine, and others with medical implants or other non-removable metal devices implanted inside their bodies may are cautioned against such scans. PET, on the other hand, is a molecular imaging process that enables visualization and assessment of metabolic processes of living cells in the body to gauge physiologic activity, including nutrient metabolism and blood flow of the organ or organs being targeted; whereas CT and MRI scans can only show static images. PET is thus widely used to diagnose conditions, such as heart disease, brain disorders, the spread of cancer, certain forms of infection, bone disease, and thyroid disease, among others [1]. Registered medical images 
can be used for diagnosis, planning therapy and monitoring disease progression or response to therapy. For instance, registering the pre-operation MRI images with an normal control MRI image could help physicians to plan the operation before intervening in the brain of the patient; registering the series of anatomy CT images obtained at different stages could help physicians find the development of the tumor without operation; registering multi-modality images, MRI with CT or MRI with PET, could integrate various information in both of the images, so physicians could take all the structural and functional information into consideration at once, and so on.

Image registration involves aligning different sets of images into a common coordinate system, which could involve both time and space alignments. It is a complex task that often requires several steps, including image pre-processing, noise removal, affine transformations, interpolation, and optimization. A comprehensive and structured record of approaches to the registration of medical images is presented in [2]. Since the review in [2] was made in 1998, a more extensive and up to date review is provided in [3], which emphasizes the shift from extrinsic registration (in relation to external objects or markers when imaging a patient) to intrinsic registration (in relation to information obtained from the patient or anatomical landmarks), the prevalence in the use of intensity-based registration methods over relying on segmentation or template matching, the advent of nonlinear registration methods, the progress in performing inter-subject registration as well, and the availability of different software packages, such as FSL and SPM that automate the process of registration. 
Methods are classified according to the different aspects of mutual-information-based registration they use as detailed in [4], which also describes aspects of preprocessing of images, gray value interpolation, optimization, adaptations to the mutual information measure, and different types of geometrical transformations. Image interpolation techniques, described in [5], are often required for image generation and processing such as resampling or compression. Several interpolation kernels of finite size have been introduced and a comparison is done. The goal in [5] was not to determine an overall best method, but to enable the reader to select an optimal method for their specific application in medical imaging. The relationship between the variations in the images and the type of most appropriate registration method to be used is given through a comprehensive survey in [6]. The three major types of variations confronted in the registration process are often due to: 1) different acquisition methods that lead to image misalignment; 2) difference in acquisition and lighting conditions; 3) differences in the images due to object movement or other scene changes. This survey is useful for understanding the merits and relationships between the wide variety of existing techniques and selecting the best technique for a specific registration problem. The problem of medical image registration for brain images is addressed in [7]. It includes a survey of recent literature, CT/MR registration using mathematical image features such as edges and ridges, mono-modal SPECT registration, and CT/MR/SPECT/PET registration using image features extracted by the use of mathematically derived morphology. A classification scheme for multimodal image matching is considered in [8]. This classification scheme involves 2D and 3D images, and it also provides spatial insight into function or anatomy/structure, electro-encephalography (EEG) and magnetoencephalography (MEG). 
A measure of regional and global cerebral volume change derived directly from registered repeat MR scans is proposed in [9]. The boundary shift integral (BSI) was used to determine brain volume loss in 21 control-scan pairs and 11 scan pairs from Alzheimer's disease patients. The potential of accurate image registration for detecting subtle changes in the brain has been tested in [10]. By the sub-voxel registration, subtle changes in the brain were detected in a variety of physiological and clinical situations. A classification of image registration by type of transformation and by methods employed to compute the transformation is provided in [11]. Two approaches to modeling soft tissue deformation for applications in image-guided interventions are described in this study. Validation of image registration is vital if the algorithms are to be used in clinical settings. An automated method to register MRI volumetric datasets to a digital human brain model is described in [12]. The non-linear registration method reduces the intersubject variability of homologous points in standardized space by $15 \%$ over linear registration methods. A general-purpose interpolation for labeled point data is developed in [13]. This method ties the geometry of image deformation to the classic biometric algebra of quadratic forms. A technique for building compact models of the shape and appearance of a flexible object such as organs seen in 2D images is described in [14]. This technique also can be simply extended to 3D object segmentation or structure tracking in image sequences.

When rotating an image, there is often some loss of image quality. To determine which function would provide the best interpolation, including nearest neighbor, linear, cubic Bspline, high-resolution cubic spline with edge enhancement, and high-resolution cubic 
spline, these five functions were compared in [15]. A new interpolation based superresolution method, named FIPOCS (Fractal interpolation with Improved Projection onto Convex Sets), is discussed in [16]. The new interpolation method shows advantages when compared to the bilinear interpolation. A new matching criterion, mutual information (MI), is applied to medical image registration in [17]. The MI is assumed to be maximal if the images are geometrically aligned. Maximizing the MI measure remains a powerful criterion because no assumptions are made regarding the nature of this dependence and no limiting constraints are imposed on the image content of the modalities involved. This study also confirmed the accuracy of the MI criterion for rigid body registration of computed tomography (CT), magnetic resonance (MR), and photon emission tomography (PET) images.

Various image registration techniques are introduced for the purpose of mapping functional activity into an anatomical image or a brain atlas. An overview of brain functional localization along with a survey and classification of the image registration techniques related to that problem is presented in [18]. Recently, a large number of medical image registration methods based on the use of metaheuristics such as evolutionary algorithms have been proposed, resulting in decisive results. The success of such methods is related to their ability to perform an effective and efficient global search in complex solution spaces. The most recognized feature-based medical image registration methods considering evolutionary algorithms and other metaheuristics are presented in [19]. An image registration method carried out by maximizing a Tsallis entropy-based divergence using a modified simultaneous perturbation stochastic 
approximation algorithm is described in [20]. This method has been demonstrated on CT, MRI, and PET images. The registration accuracy is enhanced when using this method. A new optimization method, named Big Bang-Big Crunch, which generates random points in the Big Bang phase and shrinks those points to a single representative point via a center of mass or minimal cost approach in the Big Crunch phase, is presented in [21]. This method shows superiority over an improved and enhanced genetic algorithm. A novel mutual information-based registration method that integrates the use of a Genetic Algorithm (GA), the Powell method (PM), and Wavelet decomposition in order to register in an optimal fashion the fluorodeoxyglucose (FDG)_CT and fluorodeoxythymidine (FLT)_CT image modalities are described in [22]. Registration through these tracers, FDG and FLT, increase both sensitivity and specificity for imaging cancer and is essential for optimizing the results of the diagnosis.

Nowadays, there are many mature optimization methods for image registration. Those methods could be classified into two categories: local and global. Nature selection is the principal source for the global optimization method, such as the genetic algorithm (GA) and simulated annealing (SA) method. An original usage of genetic algorithms as a robust search space sampler in an application to 3D medical image registration is presented in [23]. It focuses on the use of genetic algorithms, and particularly on the problem of extracting the optimal solution among the final genetic population. The algorithm is applied to the Vanderbilt medical image database to affirm its robustness. The suitability of GA for the model objective-function/search procedure is presented in [24]. A robust and efficient mutual information based method has been proposed in [25]. It is efficient 
as it prevents the search process from being trapped at a local maximum. A mutual information based MR and CT image registration method is presented in [26]. This study chose GA as an optimization technique and demonstrated robustness and efficiency. The point-matching problem in image registration was addressed by a nearest-neighbor based on the GA algorithm in [27]. A full implementation detail for a global optimization method is described in [28]. This method is found to be more reliable at finding the global minimum than several other existing methods. Those heuristic population-based search procedures are incorporating random variation and selection, which may result in heavy computational requirements. A novel hybrid global-local optimization method is discussed in [29]. The local optimization method, such as the Powell method (PM), is good at determining a local solution with reduced computational load but where the solution sought might not be optimal [28, 29].

The Golden Search algorithm employed by using the Powell method is discussed in [30]. The use of local optimization methods together with the standard multi-resolution approach is not sufficient to reliably find the global minimum. To address this problem, a global optimization method is proposed that is specifically tailored to this form of registration. That is why the proposed algorithm is structured to combine/integrate the strengths of both GA and PM along with the use of the wavelet decomposition method. The slice matching process was improved by calculating the Normalized Mutual Information (NMI). The registration results were enhanced by finely choosing the initial point of the second registration. The computational time of the GA has been reduced by the wavelet decomposition method. We also show that proposed algorithm avoided being 
trapped in the local best solution. The registration results achieved a non-linear registration effect by applying segmented linear interpolation. The study assumes rigid body imaging during image acquisition, although difficult to control in a clinical setting. Image acquisition at different times, breathing movements, or the position of the subject when acquiring images and so forth, all of them could affect our registration results.

How to estimate the results of medical image registration is still a problem, because no "golden estimation criterion" has been proposed. An overview of existing estimation criteria for medical image registration is presented in [31], including the advantages and shortcomings of each estimation method, it also proposes some improved methods for estimation. An investigation of similarity and dissimilarity measures and performance evaluation is given in [32].

To evaluate the registration results of the proposed method, SPM and FSL have been deployed to register the same data and compare their results. The Statistical Parametric Mapping (SPM) software package has been designed for the analysis of brain imaging data. Images are realigned, spatially normalized into a standard space, and smoothed by SPM [33]. The FMRIB Software Library (FSL) is a comprehensive library of analysis tools for fMRI, MRI and DTI brain imaging data. FLIRT is the FMRIB's linear image registration tool. It is a fully automated, robust and accurate tool for linear intra- and inter-modal brain image registration $[34,35]$. The average Normalized Mutual Information between each pair of the reference image and the registered image, and computational time of each method will be assessed in the results section. 


\subsection{General Statement of the Research Area}

This research seeks to align in time and space different imaging modalities in medical imaging through an accurate and computationally effective registration. It encompasses both brain and whole body multimodal imaging registration. The intent is to combine in one integrated algorithm methods that improve in one setting bot brain and whole body registration to attain a similar accuracy of FSL for brain registration, and SPM for whole body registration, while minimizing the computational requirements that either of these well-established software modules (FSL and SPM) would require.

\subsection{Research Purpose}

The main purpose of this research is to develop effective algorithms that will automatically register different imaging modalities to yield the required accuracy for optimal fusion of these modalities that ensure optimal diagnosis, decision making, and treatment planning. Several experiments with different medical implications are carried out to validate the merits and purpose of this research. For example, by combining the complementary strengths of the two proven tracers in FDG and FLT is shown to improve the delineation of tumors and planning of treatment; and by combining MRI and PET imaging helps in gauging the interplay between amyloid deposition observed and cortical thinning as seen in the MRI, thus helping identify early stages of Alzheimer's disease.

\subsection{Significance of the Research}

Multimodal image registration, whether it is FDG_CT and FLT_CT or MRI with PET, 
could yield validated and more meaningful information from a given patient with quantitative measures about the spatial and temporal relationship between all the image information. Aligning the spatial and temporal dimensions remains to be a challenging problem that this research aims at resolving through an integrated registration approach.

\subsection{Structure of the Research}

Chapter 2 introduces a semi-automatic registration method of FDG_CT and FLT_CT images. A brief introduction to the main method and related work is given. Then, the data and methods, including control point selection, affine transformation, and interpolation, are presented. There are four implementation aspects: 1) Minimizing errors in manual selection of control points; 2) testing the middle slice of FDG_CT from set 1 to $5 ; 3$ ) testing all slices in set 1 to 5 ; 4) performing the experimental procedure. This chapter looks also into the evaluation methods, which includes the mutual information, the normalized mutual information, and the alignment metric. Then, the experimental results are given. Finally, concluding remarks are made on this earlier research initiative.

Chapter 3 presents an automated registration method for FDG_CT and FLT_CT images. A brief introduction on the main method and related work are presented. The focus is on its two intrinsic aspects: the image pre-processing, and the procedures of the integrated Genetic-Powell-Wavelet (GPW) method. Two experiments support the improved method introduced in this Chapter, namely the verification of the Powell method and the verification of the Wavelet-Modified Genetic Algorithm. The experimental results and analysis are provided, followed by concluding remarks. 
Chapter 4 provides a more thorough investigation on the challenging steps of multimodal image registration augmented with a comparative study which contrasts the results of the proposed method with the most notable and well-established software platforms results, which are FSL and SPM. The proposed multimodal medical image registration method is hence compared to the Statistical Parametric Mapping (SPM) method that excels in whole body registration, and to the FMRIB Software Library (FSL) that excels in brain registration. The main method and related research are introduced. The datasets used in this chapter are described. The experiments conducted include: (a) the goals sought of the four different experiments, (b) a description of the implementation steps for the proposed method, and the evaluation conducted in assessing the merits of this new approach. Then, optimizing methods are discussed in detail, including the Genetic Algorithm (GA), the Powell method (PM), and the wavelet decomposition considered for optimizing the computational requirements. The registration results of the three different methods are shown juxtaposed for visual appreciation. The average normalized mutual information of the registered images are provided for evaluating the registration accuracy of each of these methods, summarizing the strengths and limitations of each method, with concluding remarks on what could be envisioned for future research to improve even further the challenging problem of registration.

Chapter 5 summarizes the dissertation, provides key remarks on what was accomplished through this research endeavor. It also summarizes the gains made when the registration is performed well. 


\section{A SEMI-AUTOMATIC REGISTRATION METHOD OF COMPUTED TOMOGRAPHY IMAGES}

\subsection{Introduction}

The widely used $18 \mathrm{~F}$-fluorodeoxyglucose (18F-FDG) serves as a good imaging tool in Computed Tomography (CT) and is essential for cancer diagnosis as FDG uptake is higher in cancerous lesions and lower in benign lesions, while 18F-fluorothymidine (FLT) uptake is closely correlated with cellular proliferation. The registration of both of these tracers, FDG and FLT, compensate one another to increase both sensitivity and specificity for imaging cancer. Consequently, accurate registration of both imaging modalities is essential for optimizing the results of the diagnosis. This chapter describes a novel feature-based registration method, which employs affine transformation and linear interpolation for FDG_CT and FLT_CT image modalities. Instead of using one set of affine transformation parameters, three slices have been selected to calculate all of the transformation parameters for $\mathrm{CT}$ image registration by linear interpolation.

This method has the merits of: a) improving the 3D registration results for CT images; $b$ ) avoiding the arbitrary selection of that one slice for calculating the transformation parameters for registration; c) being easy to realize and computationally efficient. Experimental results obtained come in support of these assertions. 


\subsection{Related Work}

Medical imaging technologies, such as CT and PET, have a significant impact on medical research and diagnostic radiology [36]. As CT is known to generate detailed images of soft tissues in the body, combining the complementary strengths of two proven tracers like FDG and FLT will only improve the outcome of the diagnosis. Thus integrating images to get more subtle information is often a requisite task in seeking such an outcome.

Image registration is the one process required for aligning different sets of data into one coordinate system in order to fuse, compare and analyze the data. Pixel values reflecting structural and functional information along with their positions should be taken into account. Image registration often includes preprocessing, affine transformations, interpolation, and overall registration process optimization [37]. Feature-based registration methods find correspondence between image features, such as points and lines. By obtaining the correspondence between a number of points in images, a transformation is then determined to map the input image to the reference image, establishing as a consequence a point-by-point correspondence between a reference image and its input counterpart [38].

An overview of medical image registration methods reveals that significant progress remains to be made towards optimized registration [37]. Feature matching methods could make use of similarity measurements as in studies [39, 40]. Mutual information based registration method using the point feature location information was developed to 
estimate the accuracy in aligning the corresponding images. A novel aspect of this method is the emergence of correspondence between the two sets of features as a byproduct of information maximization [41]. An approach, which automatically learns new corresponding landmarks from a database of 3D whole-body CT scans, using a limited initial set of expert-labeled ground-truth landmarks was presented in [42]. Based on landmark detection and calibrated camera-projector system, another technique was proposed for registration in minimally invasive spinal surgery [43]. An automated method for vertebra-based registration systems is proposed and assessed in [44].

\subsection{Methods}

\subsubsection{Control Points Selection}

As a first registration step, a set of control points $(c p)$ is chosen. The cpselect function is utilized to start the control point selection tool in MatLab. The input image and the base image are displayed in the window of the tool. A blue mark with number 1 will appear when the user clicks on the base image once, then by clicking on the corresponding point in the input image, another blue mark with number 1 will appear on it. Blue marks with number 2 form the second control-points set and so on. Four such control-points sets would complete the process. 


\subsubsection{Affine Transformation}

Affine transformation preserves points, straight lines, and planes in affine space. Parallel lines remain parallel, and the ratios of distances between points lying on a straight line are preserved. Affine transformation includes translation, rotation, and scaling. Affine transformation as applied in this study could be formulated as follows:

$$
\left(\begin{array}{l}
x \\
y
\end{array}\right)=\left(\begin{array}{ll}
a_{1} & a_{2} \\
a_{3} & a_{4}
\end{array}\right)\left(\begin{array}{l}
u \\
v
\end{array}\right)+\left(\begin{array}{l}
a_{5} \\
a_{6}
\end{array}\right)
$$

According to this model, $(x, y)$ is a point in the reference image, and $(u, v)$ is the point in the input image. Six parameters $\left(a_{1}, a_{2}, a_{3}, a_{4}, a_{5}, a_{6}\right)$ can be calculated from three pairs of set points. Therefore, to define an affine transformation, at least three points should be chosen from the input image and the reference image respectively [45].

\subsubsection{Interpolation}

Interpolation is used in this case to construct new data points within the range of a discrete set of known data points. For example, 3 sets of parameters could be obtained from the first, the middle, and the last slices pairs. Those 3 sets are the known data points. New data points can then be constructed within the range of set 1 to set 2 , as well as within the range of set 2 to set 3 by interpolation. Thus, if the total number of slices is $\mathrm{k}$, and the middle slice number is i, new data points are the parameters from slice 2 to slice (i-1), and slice (i+1) to slice $\mathrm{k}$. 
Instead of registering two CT images with a set of registration parameters, $\mathrm{k}$ sets of parameters have been used to register each pair of slices. When $\mathrm{k}$ is the total number of slices in the given $\mathrm{CT}$ image, $\mathrm{k}$ sets of parameters will thus be obtained through linear interpolation.

\section{$2.4 \quad$ Data}

This phase I pilot study includes data from five patients with resectable and unresectable pancreatic cancers who underwent 18F-FLT and 18F-FDG imaging, each acquired within a week's duration for the same patient. In these 5 datasets that have been tested, it is noted that each of them had FLT_CT and FDG_CT taken in different days. FDG_CT, in this study, is used as the reference image, which is unmoved, while the FLT_CT is used as the input image, which is moving in seeking that perfect alignment. Except for data set 1 which was of size $(512 * 512 * 186)$, all other sets were of size $(512 * 512 * 244)$ for both FDG_CT and FLT_CT image modalities.

\subsection{Experiments}

\subsubsection{Minimizing Errors in Manual Selection of Control Points}

To calculate the parameters of the first step of the registration process, control points have been chosen manually. With the manual selection process, it is difficult to visually select exactly the same point in two CT images; but choosing four vertexes of a rectangle as the assumed control points is easier as there is more contextual information. As can be 
seen in Fig. 2.1, the four vertexes of the rectangle have been marked with a "+"; thus an initial test would be that the input image could be simply obtained by rotating the reference image by 90 degrees counter-clockwise; Register those two images, and overlap the registered image with the original image. The intent here for this simple experiment is to see the marks overlap to each other with $100 \%$ accuracy. Thus, if the

control points have been chosen correctly, this registration method would yield perfect registration results.

\subsubsection{Testing the Middle Slice of FDG_CT from Set 1 to 5}

For set 1, slice No. 93 is the middle slice of the FDG_CT. That slice has been chosen as the reference image because it has more structures that can be chosen as control points than the skull. The input image has been obtained by rotating the reference image 90 degrees for visual convenience. Then register those two images. Fig. 2.2 shows this test. For set 2 to 5 , slice No. 122 has been taken as the reference image for the same reasons mentioned above.

\subsubsection{Testing All Slices in Set 1 through 5}

Select 3 pairs of slices, for example, the first slice, the middle slice, and the last slice in each set, and then register each pair in the corresponding FDG_CT and FLT_CT. This step constitutes the first part of the registration process. Using the 3 pairs of affine transformation parameters obtained thus far, determine all other parameters by linear interpolation. 


\subsubsection{Experimental Procedure}

The objective here is to register the 3D reference image FDG_CT.nii, and the 3D input image FLT_CT.nii, where nii stands for Nifti format. The procedure consists of 5 steps:

Step 1: choose the slices from each 3D image for the first registration step. For example, slice No. 1, No. i, and No. k of each 3D image have been chosen.

Step 2: choose control points manually in each slice to obtain the base points and the input points for the first part of the registration process. For affine transformations, at least 3 control-points sets should be chosen. In our study, 4 base points and 4 input points have been chosen in slice No.1 of FDG_CT and FLT_CT, respectively.

Step 3: register those pairs of slices chosen in step1 to obtain the needed affine transformation parameters. For example, if we choose 3 slices pairs, we will get 3 sets of registration parameter for set 1 , set 2 , and set 3 .

Step 4: use the affine transformation parameters determined in step 3 to calculate all of the other parameters by linear interpolation. Take $\mathrm{k}$ slices in total; for example, we use parameter set 1 and set 2 to calculate the parameters of slice No. 2 to Slice No. (i-1) $(0<\mathrm{i}<\mathrm{k})$, then we use parameter set 2 and set 3 to calculate the parameters of slice No. $(\mathrm{i}+1)$ to slice No. (k-1). Finally, all the affine transformation parameters can be obtained from slice No. 1 to slice No. k.

Steps 5: the second part of the registration process registers each pair of slices of the FDG_CT and the FLT_CT by using all the affine transformation parameter sets. 


\subsection{Evaluation Methods}

\subsubsection{Mutual Information and Normalized Mutual Information}

The mutual information $(M I)$ between image A and image $\mathrm{B}$ is:

$$
M I(A, B)=H(A)+H(B)-H(A, B)
$$

$H(A), H(B), H(A, B)$ are the entropies of image A, image $\mathrm{B}$, and the joint entropy of images $\mathrm{A}$ and $\mathrm{B}[8]$, where:

$$
\begin{gathered}
H(A)=-\sum_{a} P_{A}(a) \log _{2} P_{A}(a) \\
H(B)=-\sum_{b} P_{B}(b) \log _{2} P_{B}(b) \\
H(A, B)=-\sum_{a, b} P_{A B}(a, b) \log _{2} P_{A B}(a, b)
\end{gathered}
$$

with $a \in A, b \in B$, and $P_{A}(a), P_{B}(b), P_{A B}(a, b)$ define the probability distribution of gray values of image A, B and the joint probability distribution of gray values of images A and B. The normalized mutual information $(N M I)$ can thus be defined as:

$$
N M I=\frac{M I}{\sqrt{H(A) * H(B)}}
$$

From here onward, this is the NMI measure used throughout this dissertation to gauge the accuracy of the registration process. 


\subsubsection{Alignment Metric}

Images $I_{1}(x, y)$ and $I_{2}(x, y)$ are $M \times N$ images, and $H_{1}(n)$ and $H_{2}(n)$ are their corresponding histograms. The gray scale of the image is $0 \leq n \leq 255$. If $n=i, H_{1}(n)$ and $H_{2}(n)$ are the total number of pixels whose gray value is $i$ in $I_{1}(x, y)$ and $I_{2}(x, y)$. The ratios of gray value $i$ in image $I_{1}(x, y)$ and $I_{2}(x, y)$ are determined as follows:

$$
\begin{aligned}
& p_{1}(i)=H_{1}(i) /(M \times N) \\
& p_{2}(i)=H_{2}(i) /(M \times N)
\end{aligned}
$$

For each gray scale $\mathrm{n}$ in image $I_{1}(x, y), H_{1}(n)$ is the total number of pixels whose gray value is $\mathrm{n}$, Determine next the coordinates of those pixels in $I_{1}(x, y)$, using the same coordinates to search for those same pixels in image $I_{2}(x, y)$ and sum the gray values in those positions, then we could get the mean vector $\bar{E}_{1,2}(n)$ and the relative variance $\sigma_{1,2}^{2}(n)$ as follows:

$$
\begin{gathered}
\bar{E}_{1,2}(n)=\frac{1}{H_{1}(n)} \sum_{I_{1}(x, y)=n} I_{2}(x, y) \\
\sigma_{1,2}^{2}(n)=\frac{1}{H_{1}(n)} \sum_{I_{1}(x, y)=n}\left(I_{2}(x, y)-\bar{E}_{1,2}(n)\right)^{2}
\end{gathered}
$$

Similarly, for each gray scale $\mathrm{n}$ in image $I_{2}(x, y), H_{2}(n)$ defines the total number of pixels whose gray value is $\mathrm{n}$, Similarly, determine the coordinates of those pixels in $I_{2}(x, y)$, and using these same coordinates, search for the pixels in image $I_{1}(x, y)$ and 
sum the gray values in those positions, then we could get the mean vector $\bar{E}_{2,1}(n)$ and the relative variance $\sigma_{2,1}^{2}(n)$ as follows:

$$
\begin{gathered}
\bar{E}_{2,1}(n)=\frac{1}{H_{2}(n)} \sum_{I_{2}(x, y)=n} I_{1}(x, y) \\
\sigma_{2,1}^{2}(n)=\frac{1}{H_{2}(n)} \sum_{I_{2}(x, y)=n}\left(I_{1}(x, y)-\bar{E}_{2,1}(n)\right)^{2}
\end{gathered}
$$

These variances based on $I_{1}(x, y)$ and on $I_{2}(x, y)$ can be determined as follows:

$$
\begin{aligned}
& \bar{\sigma}_{1,2}^{2}=\sum_{n} p_{1}(n) \sigma_{1,2}^{2}(n) \\
& \bar{\sigma}_{2,1}^{2}=\sum_{n} p_{2}(n) \sigma_{2,1}^{2}(n)
\end{aligned}
$$

Let's define the cross variance $(\mathrm{CI})$ based on $I_{1}(x, y)$ and $I_{2}(x, y)$ as given below:

$$
C I\left[I_{1}, I_{2}\right]=\frac{\bar{\sigma}_{1,2}^{2}}{\sigma_{2}^{2}}+\frac{\bar{\sigma}_{2,1}^{2}}{\sigma_{1}^{2}}
$$

where, $\mu_{1}$ and $\mu_{2}$ are the mean values, and where $\sigma_{1}^{2}$ and $\sigma_{2}^{2}$ are the variance of image $I_{1}(x, y)$ and $I_{2}(x, y)$, respectively.

$$
\begin{aligned}
& \sigma_{1}^{2}=\frac{1}{M N} \sum_{(x, y)}\left(I_{1}(\mathrm{x}, \mathrm{y})-\mu_{1}\right)^{2} \\
& \sigma_{2}^{2}=\frac{1}{M N} \sum_{(x, y)}\left(I_{2}(\mathrm{x}, \mathrm{y})-\mu_{2}\right)^{2}
\end{aligned}
$$

Obviously, $\sigma_{1}^{2}$ and $\sigma_{2}^{2}$ are constants. The better images are registered, the smaller are the $\bar{\sigma}_{1,2}^{2}$ and $\bar{\sigma}_{2,1}^{2}$ values, therefore the smaller is the CI measure. We could thus define the 
alignment metric ( $\mathrm{AM}$ ) as below, where the $\mathrm{AM}$ would consequently increase as the two images are registered better.

$$
A M\left(I_{1}, I_{2}\right)=\frac{1}{C I\left[I_{1}, I_{2}\right]}
$$

\subsection{Results}

Fig. 2.1 shows the control markers, which as expected are almost perfectly overlapping with each other. Minimal errors are still observed however for the rectangle as shown in Table 2.1 to indicate that manual selection of the control points is still difficult to perfect even under visual scrutiny. Table 1 provides the computed MI, NMI, and AM measurements, which clearly prove the soundness of the proposed registration method between the output image and the reference image.

Fig. 2.2 shows the results when overlapping the output image to the reference image. According to the results in Table 1, when the input image is exactly the same as the reference image, the average of maximum MI, NMI, and AM are 1.65178, 0.49938, and 17.72104, respectively. The MI, NMI, and AM are higher when FDG_CT and FLT_CT have been registered.

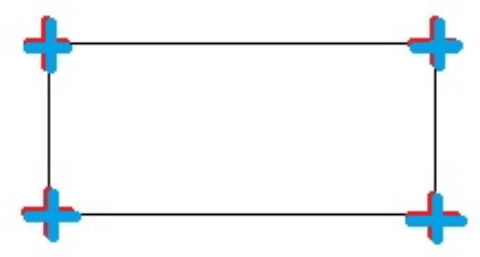

Fig. 2.1 The registered image with 4 blue "+" marks overlapping the reference image with 4 red "+" marks. 


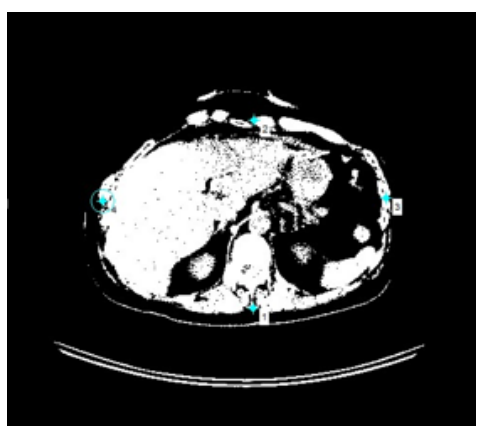

(a)

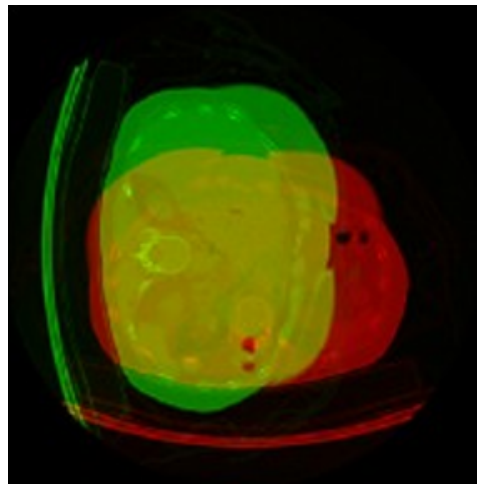

(c)

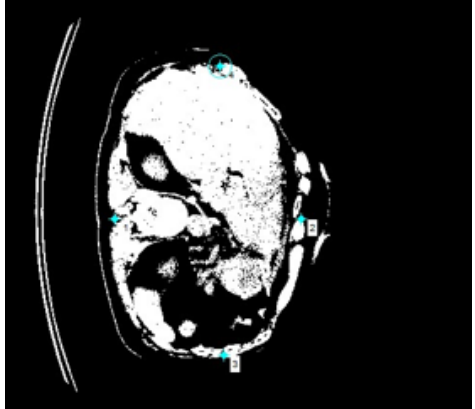

(b)

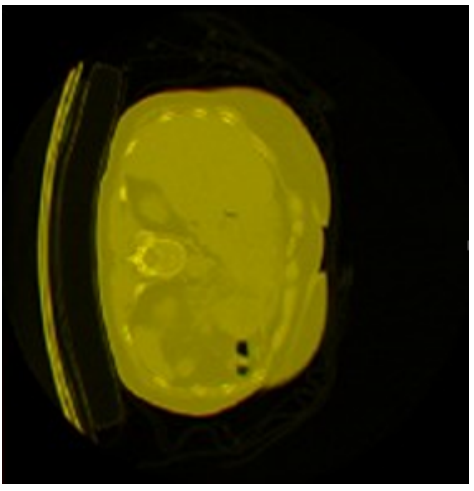

(d)

Fig. 2.2 Images (a) and (b) are the input image and the reference image with four blue control points marked in each of them. Image (c) is overlapping the green reference image, slice No. 93 of FDG_CT of set 1, and the red input image which is rotating the green image 90 degree counter-clockwise. Image (d) is overlapping the output image and the reference image.

Table 2.1 Evaluation for sets 1 through 5: $\mathrm{I}$ is the input image, $\mathrm{R}$ is the reference image, and $\mathrm{O}$ is the registered FLT_CT image

\begin{tabular}{|c|c|c|c|c|c|c|}
\hline DATA & \multicolumn{2}{|c|}{ SET 1 } & \multicolumn{2}{c|}{ SET 2 } & \multicolumn{2}{c|}{ SET 3 } \\
\hline IMAGES & I \& R & O \& R & I \& R & O \& R & I \& R & O \& R \\
\hline MI & 0.7199 & 1.9108 & 0.7647 & 1.6365 & 0.6548 & 1.6894 \\
\hline NMI & 0.2065 & 0.5472 & 0.2140 & 0.4576 & 0.2061 & 0.5369 \\
\hline AM & 0.9451 & 23.0493 & 1.0930 & 13.7417 & 0.7233 & 20.0637 \\
\hline DATA & \multicolumn{2}{|c|}{ SET 4 } & \multicolumn{2}{c|}{ SET 5 } & \multicolumn{2}{c|}{ RECTANGLE } \\
\hline IMAGES & I \& R & O \& R & I \& R & O \& R & I \& R & O \& R \\
\hline MI & 0.6548 & 1.5133 & 0.7374 & 1.5089 & 0.0007 & 0.2300 \\
\hline NMI & 0.2061 & 0.4776 & 0.2326 & 0.4776 & 0.0029 & 0.9094 \\
\hline AM & 0.7233 & 11.8124 & 1.1149 & 19.9381 & 0.5022 & 26.9311 \\
\hline
\end{tabular}


Fig. 2.3 Illustrates the results obtained by registering the 3 selected slices (using, in this case, the first, the middle, and last slices) in the first part of the registration process.

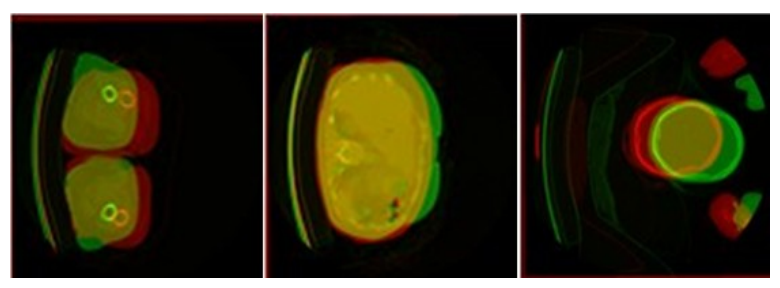

(a)

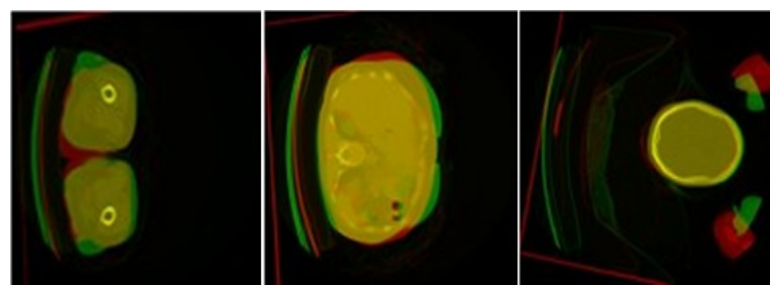

(b)

Fig. 2.3 Set 1: 3 slices for the first registration. Images (a) are the first, the middle, and last of the unregistered slices; Images (b) show the registered slices.

Fig. 2.4 shows the plots the AM measurements for all 5 sets for visual appreciation, and to see how these measurements vary depending on which parts of the body these slices belong to.

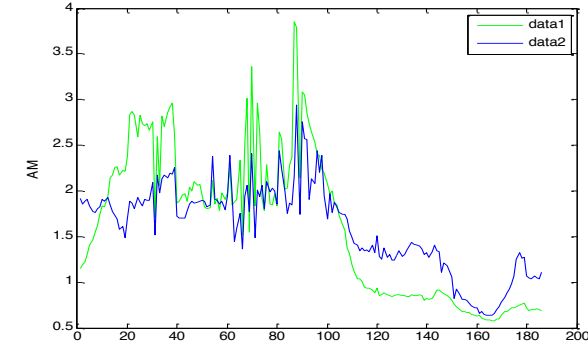

(a) Set 1

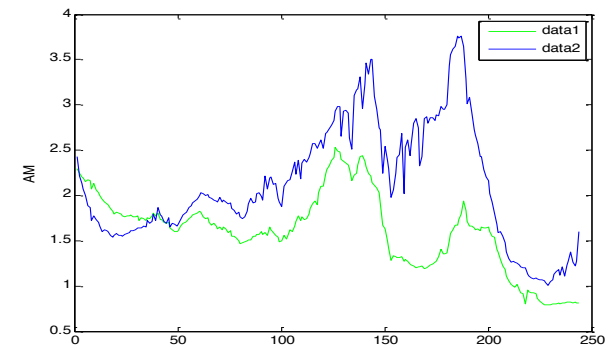

(b) Set 2 


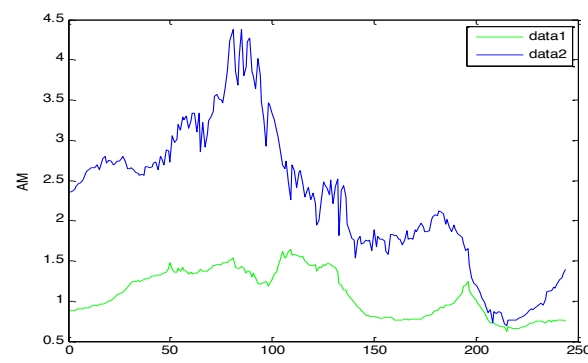

(c) Set 3

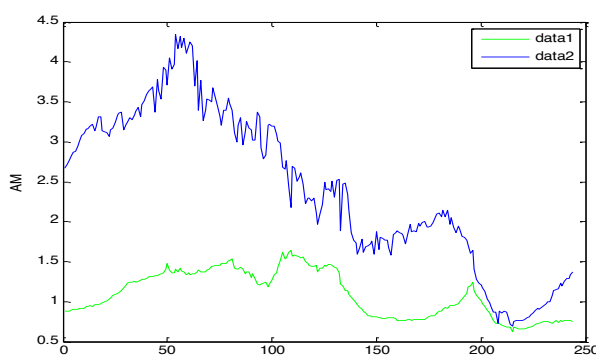

(d) Set 4

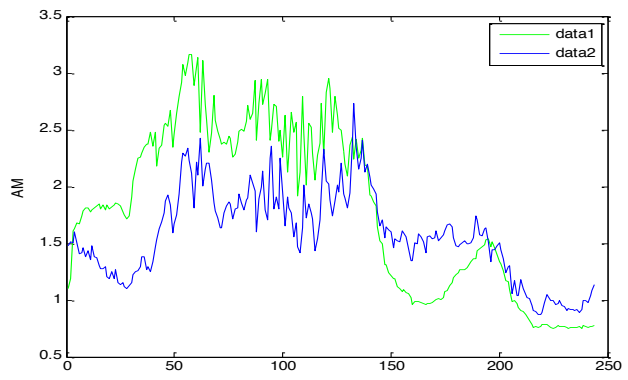

(e) Set 5

Fig. 2.4 The AM for set 1 to 5. Green data 1 is the AM of FLT_CT and FDG_CT; Blue data 2 is the AM of registered image and FDG_CT. Blue data is higher than green data (registration improved) in these parts: (a) from slice No.1 to slice No.11 and from slice No. 105 to slice No.186; (b) from slice No. 40 to slice No. 244; (c) and (d) all of the slices;(e)from slice No. 139 to slice No. 244 in set 5.
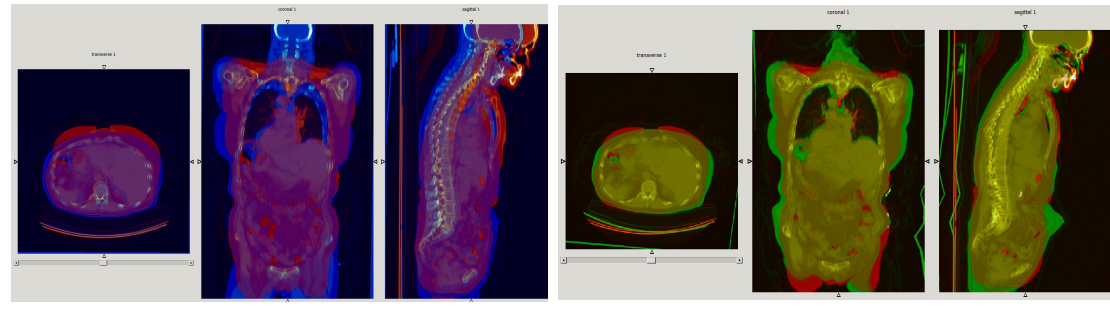

(a) Set 1 

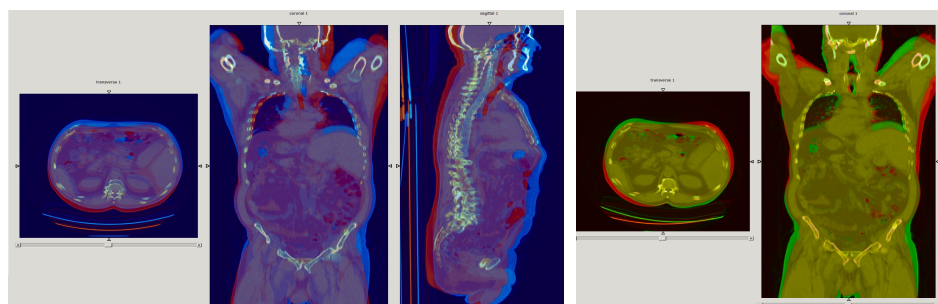

(b) Set 2
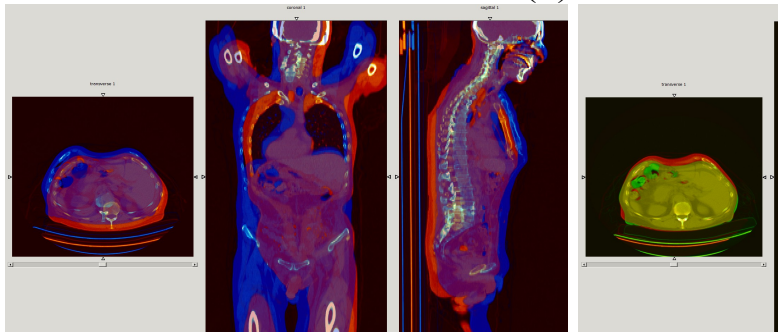

(c) Set 3
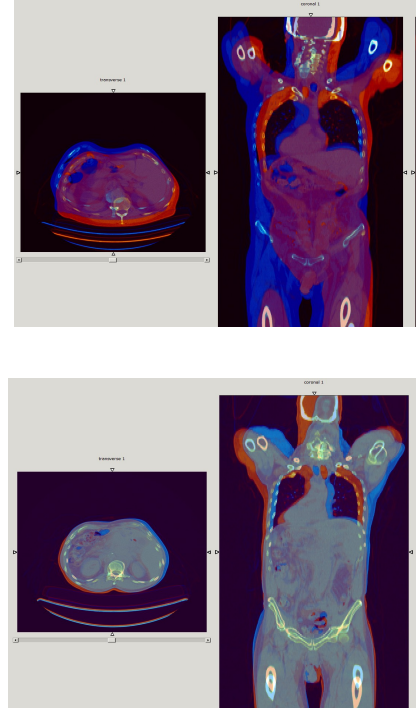
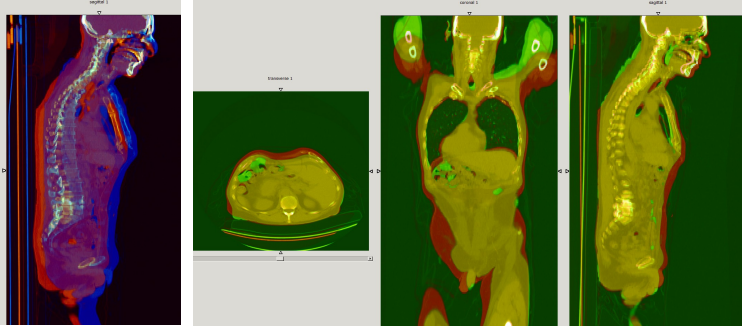

(d) Set 4
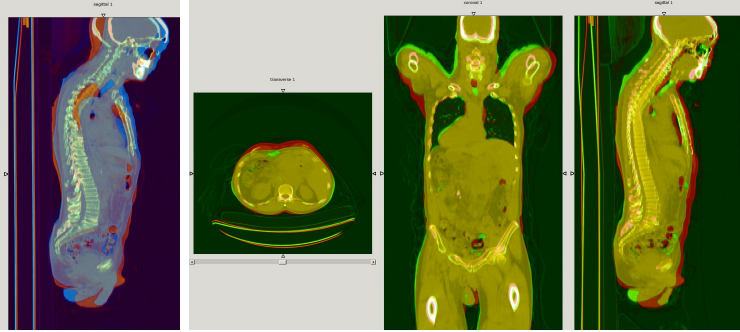

(e) Set 5

Fig. 2.5 Set 1 to 5: Overlapped the unregistered blue FLT_CT and red FDG_CT; overlapped are the registered green FLT_CT and red FDG_CT. These exemplify typical slice and whole body registration for all $\overline{5}$ patients

Fig. 2.5 Provides the results of the overall registration process illustrated through a typical dataset, with similar good results obtained for the other four datasets. For the visual appreciation of the merits of these registration results, focus on the ossature frame, and more specifically on the vertebrae as shown in the FDG_CT and their counterpart in the FLT_CT. Recall that these image modalities were obtained on different days. 


\subsection{Conclusion}

To sum up, the method as proposed improved the CT registration of the FDG_CT and FLT_CT imaging modalities, in support of combining the strengths of both tracers in cancer diagnosis. The registration of these slices provides a comprehensive 3D wholebody combined FDG-FLT CT image. To avoid arbitrarily using one set of affine transformation parameters to register images, three (first, middle and last) slices were initially used to calculate the whole set of transformation parameters for the entire 3D whole body CT images through linear interpolation. Improvements could be observed obviously in the skull and the bottom parts of the whole body CT.

Computationally, this method can accomplish the registration of two $512 * 512 * 244$ bit CT images in about 1 minute of processing time using MatLab on a Windows 7 workstation with $3.40 \mathrm{GHz}$ Intel (R) Core (TM) i7-2600 CPU and 4GB RAM. Experimental results clearly demonstrate the soundness of the proposed method in terms of both small registration errors as indicated in Table 2.1 as well as and in terms of visual scoring or appreciation. 


\section{AUTOMATIC REGISTRATION OF FDG_CT AND FLT_CT \\ IMAGES}

\subsection{Introduction}

This chapter describes a novel mutual information-based registration method that integrates the use of a Genetic Algorithm (GA), the Powell method (PM), and Wavelet decomposition in order to register in an optimal fashion the 18fluorodeoxyglucose (FDG)_CT and 18fluorodeoxythymidine (FLT)_CT image modalities. By registering these two computed tomography (CT) modalities, we combine the strengths of the two radiotracers knowing that FDG uptake is higher in cancerous lesions, while FLT uptake is closely correlated with cellular proliferation.

Registration through these tracers, FDG and FLT, increase both sensitivity and specificity for imaging cancer and is essential for optimizing the results of the diagnosis. In this study, this integrated approach, which combines the Genetic Algorithm, Powell method augmented through the Wavelet decomposition, we refer to as the GPW method, focuses

on solving three problems: (1) Reducing the computational time of GA required when it is searching for the best global solution; (2) Preventing the Powell method (PM) method to fall into a local solution while performing image registration; (3) Providing the necessary image pre-processing steps for enhanced feature analysis of FDG_CT and FLT_CT images. After registration, the location of the cancerous lesions on the liver could be observed directly on the FLT_CT image. When registering wavelet decomposed 
images, the GA is used for determining the maximal value of the NMI between a reference image and a moving image, while the Powell method (PM) is implemented in search for the best solution starting from an initial set of registration points.

\subsection{Related Work}

Computed Tomography (CT) continues to yield a significant impact on medical research and remain one of the viable imaging modality for diagnosis [46]. Combining the complementary strengths of two proven tracers FDG and FLT is an effective way to improve diagnosis, overcoming their inherent limitations when used separately.

As indicated earlier, image registration is a subtle and yet complex task that often require several steps that include image pre-processing, use of affine transformations, interpolation, similarity metrics, and optimization [47]. This involves a thorough assessment of the feature space, determining what similarity metrics should be used to gauge the mutual information and frame a search strategy that will optimize the registration process in terms of both accuracy and computational simplicity.

An overview of different image registration methods reveals that significant progress remains to be made towards a more effective solution to registration [48]. William M. Wells III et al. proposed a registration method that was achieved by adjusting the relative position and orientation until the mutual information between the images is maximized [49]. Lisa Tang and her colleagues offer a registration method that was focused on

optimizing the mutual information [50]. X. F. Wang et al. use a genetic-based image 
registration method [51]. X. G. Du et al. propose instead a multi-modal medical image registration method based on the gradient of mutual information and hybrid genetic algorithm [52]. X. Du et al. describe a multi-resolution image registration method based on the so-called firefly algorithm and Powell method [53].

Genetic algorithms [51, 52] and Powell method (PM) [53, 54] remain the most popular optimization methods applied to mutual information-based medical image registration. Genetic algorithms are often used when seeking the best global solution within the whole range, which may result in heavy computational requirements; while the Powell method is good at determining a local solution with reduced computational load but where the solution sought might not be optimal.

In order to take the advantage of both GA and PM, an automatic registration method combining the strength of each, augmented with wavelet decomposition (GPW) is proposed in this Chapter, considered as the main contribution of this dissertation.

\subsection{Methods}

\subsubsection{Image Pre-processing Methods}

The image preprocessing steps assumed throughout this chapter can be summarized as outlined in Fig. 3.1. These pre-processing steps include image normalization, median filtering, Laplacian shaping, and histogram enhancement. Assume the pixel value at point $(\mathrm{x}, \mathrm{y})$ to be $\mathrm{p}(\mathrm{x}, \mathrm{y})$, and where $\min [\mathrm{p}(\mathrm{x}, \mathrm{y})]$ and $\max [\mathrm{p}(\mathrm{x}, \mathrm{y})]$ are the minimum and 
maximum of $\mathrm{p}(\mathrm{x}, \mathrm{y})$, respectively, then expression $\{\mathrm{p}(\mathrm{x}, \mathrm{y})-\min [\mathrm{p}(\mathrm{x}, \mathrm{y})]\} /\{\max [\mathrm{p}(\mathrm{x}, \mathrm{y})]$ $-\min [\mathrm{p}(\mathrm{x}, \mathrm{y})]\}$ is used to normalize the original image. Median filtering is performed on the image using the default 3-by-3 neighborhood mask, to remove isolated or spurious noise points. Laplacian shaping removes the low-frequency components while keeping the high-frequency components in the Fourier domain. The edges thus become much more recognizable than in the original image. Histogram enhancement is applied to the Laplacian sharpened images in order to observe more details if needed. Fig. 3.2 shows the results of the pre-processing steps.

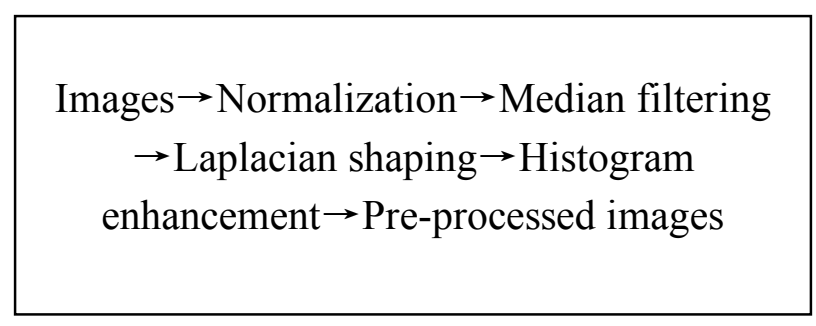

Fig. 3.1 Pre-processing steps 


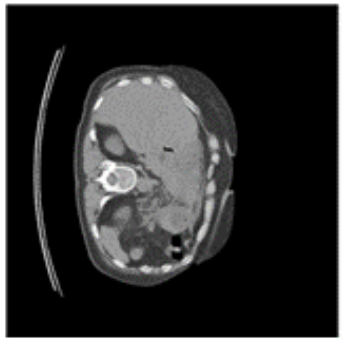

(a) Reference image: FDG_CT

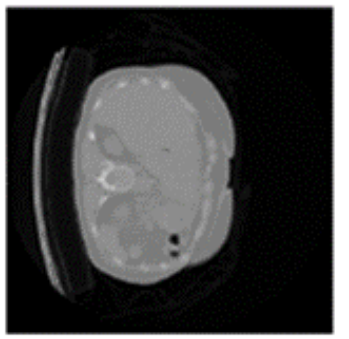

(c) Normalization results
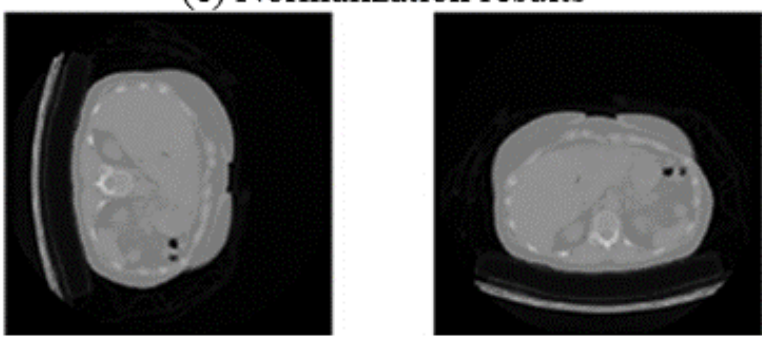

(d) Median filtering results
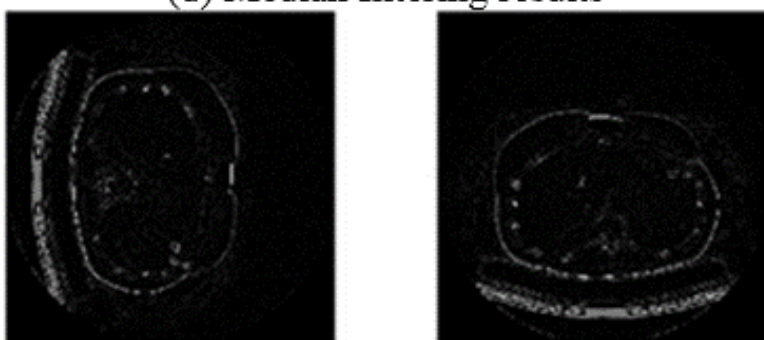

(e) Laplacian shaping results
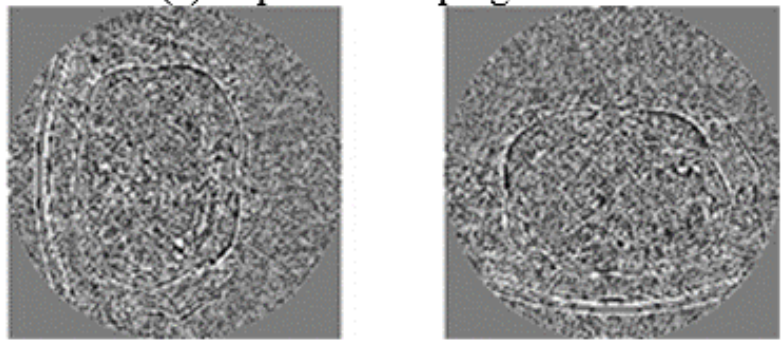

(f) Histogram enhancement results

Fig. 3.2 Results of pre-processing procedure 


\subsubsection{The procedures of GPW Method}

The flow diagram of the GPW structure is shown in Fig.3.3. FDG_CT and FLT_CT are pre-processed as $512 * 512$ images.

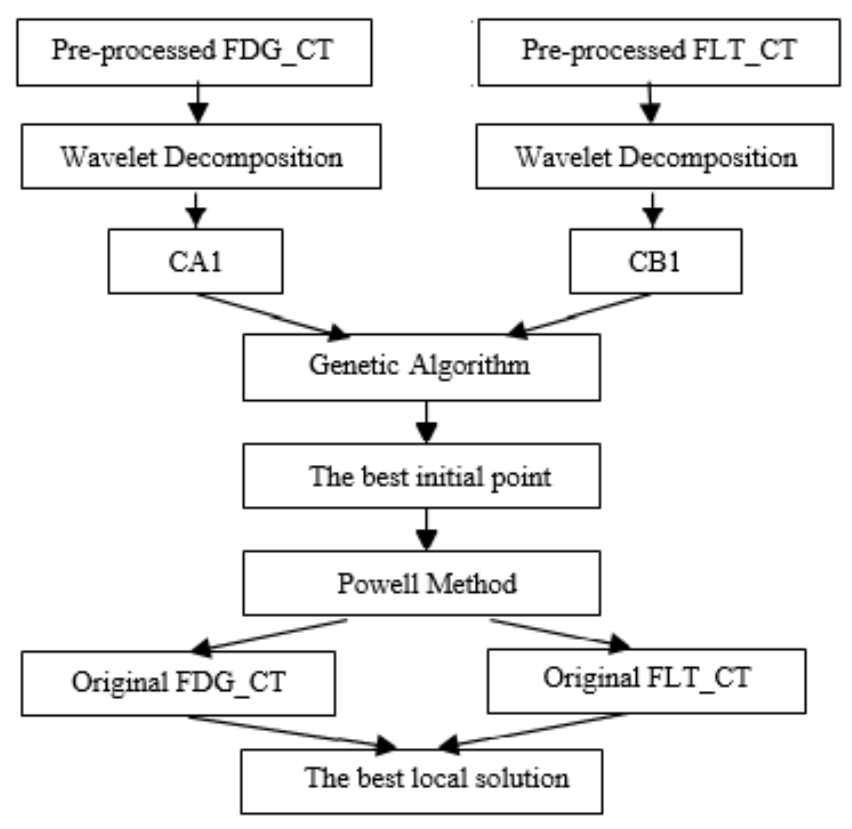

Fig. 3.3 The GPW integrated structure

First, the wavelet method is applied to both images, yielding two decomposed $256 * 256$ images (CA1 and CB1). CA1 and CB1 are the low-frequency parts of FDG_CT and FLT_CT, respectively. The processing time was thus reduced significantly since the size of the images is decreased by half both dimensions from $512 * 512$ to $256 * 256$.

Second, the GA is used to search for the best global solution in the registration process and is applied only on the $256 * 256 \mathrm{CA} 1$ and CB1, which are smaller than the preprocessed FDG_CT and FLT_CT images. Furthermore, to save time, the size of the 
image could be further decomposed several times depending on the requirements and the application at hand.

Thirdly, the best global solution found by the GA is fed as an initial solution (point) to the Powell method (PM), which is applied to register the original 512*512 FDG_CT and FLT_CT images. Setting the initial point properly is very important when using PM; with the initial point being the global best here, searching near this point, PM could find the best local solution quickly.

The GA and PM algorithms are applied to the original FDG_CT images as described in Fig. 3.4 and Fig. 3.5.

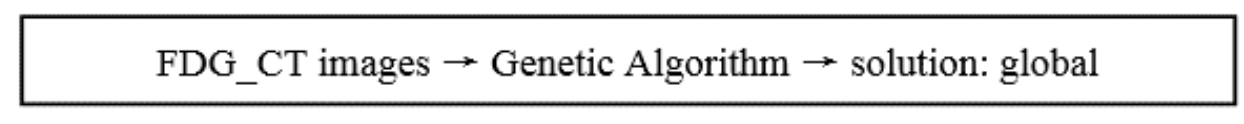

Fig. 3.4 Registration with GA optimization

FDG_CT images $\rightarrow$ Powell Method $\rightarrow$ solution: global or local solution

Fig. 3.5 Registration with PM optimization

\subsection{Experiments}

\subsubsection{Performance Verification of the Powell Method (PM)}

To verify that the Powell method has worked properly, the following experiments were conducted: Slice No. $93(512 * 512)$ of FDG_CT used as the reference image, and its 
rotated version by 90 degrees anti-clockwise serves as the moving image that is going to be registered to the reference image. The initial point of Powell method was set randomly, and the experiment was repeated 10 times with the results as shown in Table 3.1.

Table 3.1 Registration results of PM

\begin{tabular}{|c|c|c|c|c|c|c|c|c|}
\hline$\#$ & $\begin{array}{c}\mathbf{X} \\
\text { (pixels) }\end{array}$ & $\begin{array}{c}\mathbf{Y} \\
\text { (pixels) }\end{array}$ & $\begin{array}{c}\text { Angle } \\
\text { (degrees) }\end{array}$ & NMI & MI & $\begin{array}{c}\text { T } \\
\text { (seconds) }\end{array}$ & Local & Global \\
\hline 1 & -2.00 & -3.4 & -90.0 & 0.999 & 4.569 & 341 & 0 & 1 \\
\hline 2 & -2.85 & -0.7 & -89.7 & $\mathbf{0 . 1 8 6}$ & 0.850 & 549 & $\boldsymbol{1}$ & 0 \\
\hline 3 & 0.25 & 0 & -349.8 & $\mathbf{0 . 0 9 8}$ & 0.449 & 5783 & $\boldsymbol{1}$ & 0 \\
\hline 4 & -2.00 & 0 & -90.0 & 0.999 & 4.568 & 423 & 0 & 1 \\
\hline 5 & -2.00 & 0 & -90.0 & 0.999 & 4.569 & 321 & 0 & 1 \\
\hline 6 & -2.00 & 0 & -90.0 & 0.997 & 4.559 & 423 & 0 & 1 \\
\hline 7 & -2.00 & 0 & -90.0 & 0.999 & 4.566 & 519 & 0 & 1 \\
\hline 8 & -2.00 & 0 & -90.0 & 0.997 & 4.559 & 437 & 0 & 1 \\
\hline 9 & -2.00 & 0 & -90.0 & 0.999 & 4.566 & 541 & 0 & 1 \\
\hline 10 & -2.00 & 0 & -90.0 & 0.997 & 4.559 & 438 & 0 & 1 \\
\hline
\end{tabular}

According to the results of Table 3.1, experiment \#1, and \#4 to \#10 achieved a normalized mutual information higher than 0.99 , which means the registration is correct above $99 \%$, which is the near perfect results that were expected at this point. And the average computational time is thus far 431 seconds for one slice. If the total slice number is 186 , the approximate total computational time will be close to 22 hours. So PM did find the global best solution in those eight experiments. But in experiment \#2 and \#3, PM 
fell into the local best solution. Thus, the success rate of registration using PM optimization is $80 \%$ ( 8 out 10 ) in this set of experiments.

This outcome highlights the importance of the initial point when applying the PM, as there are possibilities for failure, as was the case in experiments \#2 and \#3. Assuming setting the initial point right at or close to the global best point, PM may on the other lead to the right solution and faster. Since the GA is good at finding the best global solution, GA is assumed to optimize the search for a solution first, and then passing this best global point to the PM as the initial point.

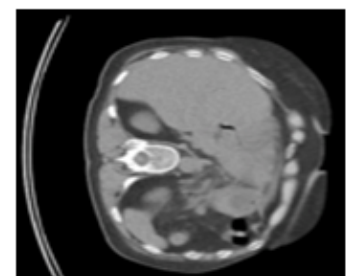

(a) Reference image: FDG_CT

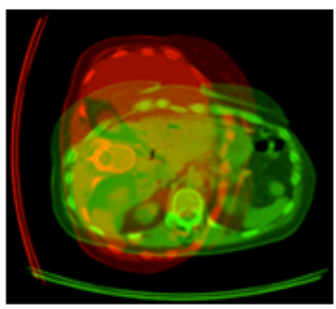

(c) Fusion image before registration

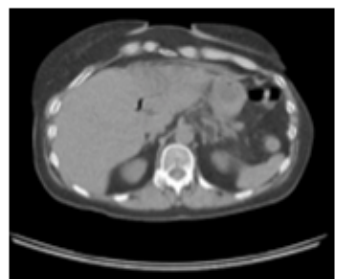

(b) Moving image: 90 degree Anti- clockwise FDG_CT

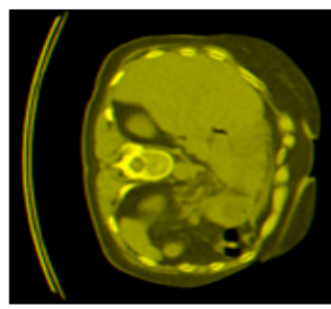

(d) Fusion image after registration

Fig. 3.6 Registration results of experiment \# 1 using PM

Fig. 3.6 shows the registration results of experiment \#1: reference image (FDG_CT), moving image (90 degrees anti-clockwise FDG_CT), and the fusion of images before and after registration. For visual convenience, the reference image was put into the red 
channel, and the registered image was put into the green channel. The yellow points show the overlapping parts of them after registration. The registration result is excellent in this case since the whole fusion image after registration turns into yellow.

\subsubsection{Verification of Wavelet-Modified Genetic Algorithm (GA)}

In this experiment, slice N0.93 $(512 * 512)$ of FDG_CT is also used as the reference image, and its rotated version by 90 degrees anti-clockwise serves as the moving image. Wavelet decomposition is applied to the $512 * 512$ image to get the low-frequency part image $(256 * 256)$ and three high-frequency parts of it. And then, wavelet decomposition is applied to the low-frequency part $(256 * 256)$ one more time to get a second level lowfrequency part image $(128 * 128)$. The experiment here is about using GA to optimize the solution in the registration of the $512 * 512,256 * 256$, and $128 * 128$ images, respectively. The initial point and conditions of GA are set to be the same when registering these 3 sets of different size images. The registration results are as recorded in Table 3.2.

Table 3.2 Registration results of GA

\begin{tabular}{|c|c|c|c|c|c|c|c|}
\hline $\begin{array}{c}\text { Image Size } \\
\text { (pixels) }\end{array}$ & $\begin{array}{c}\mathbf{X} \\
\text { (pixels) }\end{array}$ & $\begin{array}{c}\mathbf{Y} \\
\text { (pixels) }\end{array}$ & $\begin{array}{c}\text { Angle } \\
\text { (degrees) }\end{array}$ & NMI & $\begin{array}{c}\text { T1 } \\
\text { (seconds) }\end{array}$ & $\begin{array}{c}\text { T2 } \\
\text { (seconds) }\end{array}$ & $\begin{array}{c}\text { T } \\
\text { (seconds) }\end{array}$ \\
\hline $512 * 512$ & 1.99 & 0.0038 & -90.002 & 0.978 & 0 & 3833 & 3833 \\
\hline $256 * 256$ & 2.01 & -0.0125 & -90.013 & 0.944 & 0.033 & 960 & 960.03 \\
\hline $128 * 128$ & 2.00 & -0.0001 & -90.001 & 0.992 & 0.037 & 244 & 244.04 \\
\hline
\end{tabular}


According to the results given in Table 3.2, T1 was the processing time of applying wavelet decomposition to the $512 * 512$ images once to get the $256 * 256$ images. T2 was the computational time of GA when GA was applied to the original 512*512 FDG_CT image, where the computational time was 3833 seconds. As the original image has been wavelet decomposed to level one, the size of the image has been decreased to $256^{*} 256$, at the same time, the computational time went down to 960 seconds, which was 2873 seconds less than before. Then the second level wavelet decomposition was applied to the $256 * 256$ images, where the computational time went down to 244 seconds, which was 716 seconds less than before, meanwhile, this time achieved the highest normalized mutual information 0.992 . Furthermore, the processing time of wavelet decomposition to get the first level image and the second level image were 0.033 seconds and 0.037 seconds, respectively, which are negligible. With respect to the whole algorithm, these processing times could as well be ignored. This experiment proved that by using wavelet decomposition, the processing time could be significantly reduced, while the GA still ensures an optimized image registration process.

\subsection{Results and Analysis}

In this experiment, seven pairs of FDG_CT and FLT_CT images were registered using the integrated GPW approach. Slice No. 92 to slice No. 98 of FDG_CT images and slice No. 92 to No. 98 of FLT_CT images were tested. Wavelet decomposition was applied twice to the pre-processed $512 * 512$ FDG_CT and FLT_CT images to get the $128 * 128$ images as the reference and moving images. GA is used to obtain the best initial point to be used by PM for optimizing the registration results on the original 512*512 images. 
Results provided in Table 3.3 show that the total computational time (T3+T4) for registering slice No. 92 through slice No. 98 by GPW are: 610 seconds, 706 seconds, 945 seconds, 928 seconds, 623 seconds, 628 seconds, and 518 seconds, respectively. The average computational time for registering a pair of 512*512 FDG_CT and FLT_CT image using GPW is 708 seconds. It is 3556 seconds less than registering a pair of 512*512 image by GA (average computational time is 3833 seconds) plus PM (average computational time is 431 seconds). The NMIs of those seven tests were $0.3006,0.2747$, $0.3032,0.2391,0.2212,0.2254$, and 0.2378 , respectively. Those NMIs were low, but that does not mean that the registration results were poor; in fact, the original images used for registration here were different. So the NMIs cannot possibly approach 1. But the goal here was to compare the differences between the FDG_CT and FLT_CT images. So the goal was still achieved and is validated through visual observation.

Table 3.3 Register FDG_CT and FLT_CT images by GPW

\begin{tabular}{|c|c|c|c|c|c|c|c|c|}
\hline \multirow{3}{*}{ \# } & \multicolumn{5}{|c|}{ GA } & \multicolumn{3}{|c|}{ PM } \\
\hline & \multirow{2}{*}{$\begin{array}{c}\text { Size } \\
\text { (pixels) }\end{array}$} & \multicolumn{3}{|c|}{ Initial point } & \multirow{2}{*}{$\begin{array}{c}\text { T3 } \\
\text { (seconds) }\end{array}$} & \multirow{2}{*}{$\begin{array}{c}\text { Size } \\
\text { (pixels) }\end{array}$} & \multirow[b]{2}{*}{ NMI } & \multirow{2}{*}{$\begin{array}{c}\text { T4 } \\
\text { (seconds) }\end{array}$} \\
\hline & & $\underset{\text { (pixels) }}{\mathbf{X}}$ & $\begin{array}{c}\mathbf{Y} \\
\text { (pixels) }\end{array}$ & $\begin{array}{c}\text { Angle } \\
\text { (degrees) }\end{array}$ & & & & \\
\hline 92 & $128 * 128$ & -8.1 & -4.01 & -0.01 & 285 & $512 * 512$ & 0.3006 & 325 \\
\hline 93 & $128 * 128$ & -7.0 & -4.01 & -0.01 & 298 & $512 * 512$ & 0.2747 & 408 \\
\hline 94 & $128 * 128$ & -7.0 & -2.01 & 0.04 & 292 & $512 * 512$ & 0.3032 & 653 \\
\hline 95 & $128 * 128$ & -8.9 & -2.5 & 0.05 & 301 & $512 * 512$ & 0.2391 & 627 \\
\hline 96 & $128 * 128$ & -8.0 & -2.5 & -0.08 & 295 & $512 * 512$ & 0.2212 & 328 \\
\hline 97 & $128 * 128$ & -9.1 & -2.0 & 0.05 & 297 & $512 * 512$ & 0.2254 & 331 \\
\hline 98 & $128 * 128$ & -10.0 & -2.5 & -0.01 & 297 & $512 * 512$ & 0.2378 & 221 \\
\hline
\end{tabular}




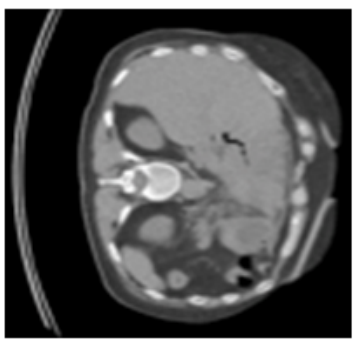

(a) Slice No. 92 FDG CT

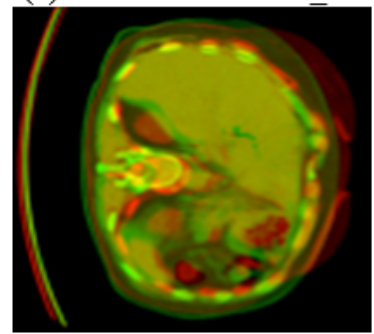

(c) Fusion image before registration

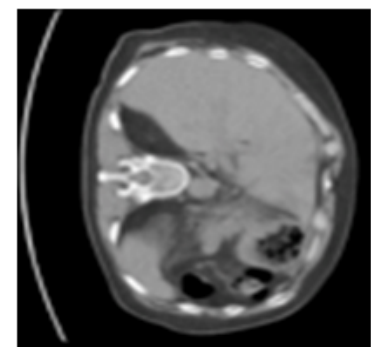

(b) Slice No. 92 FLT CT

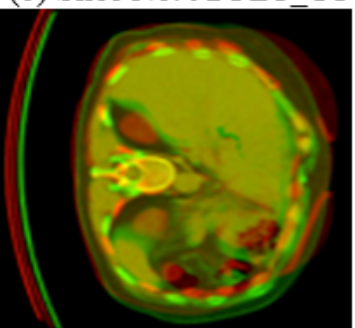

(d) Fusion image after registration

Fig. 3.7 Registration results of slice No.92 FDG_CT and FLT_CT image

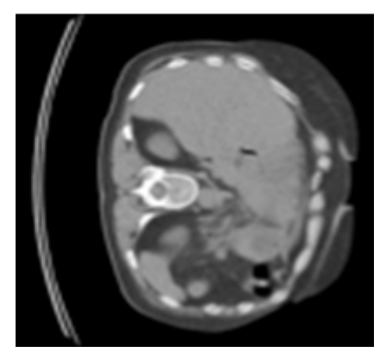

(a) Slice No. 93 FDG_CT

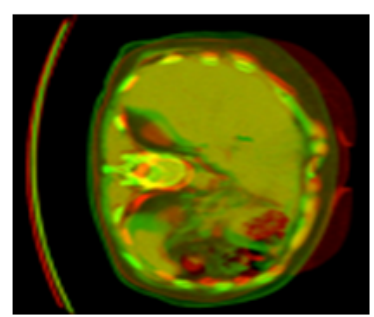

(c) Fusion image before registration

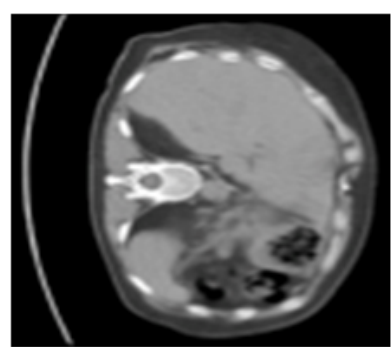

(b) Slice No. 93 FLT_CT

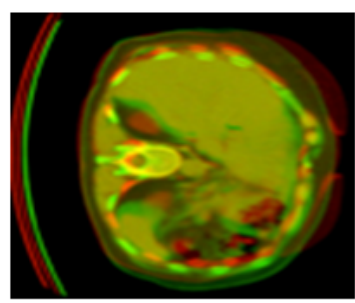

(d) Fusion image after registration

Fig. 3.8 Registration results of slice No.93 FDG_CT and FLT_CT image 


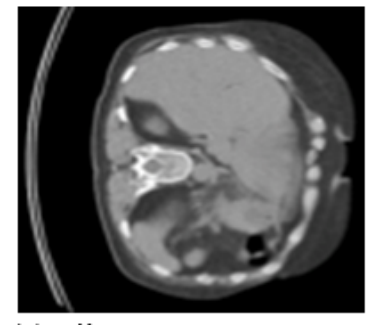

(a) Slice No. 94 FDG_CT

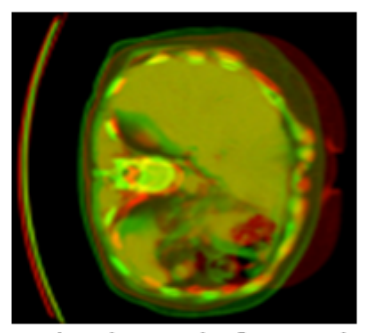

(c) Fusion image before registration

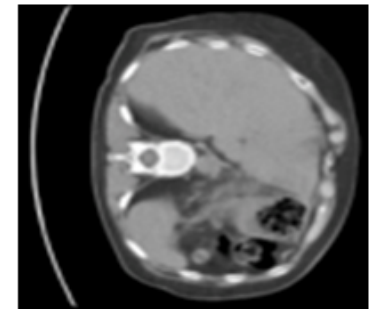

(b) Slice No. 94 FLT_CT

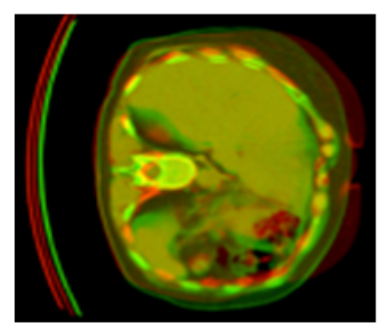

(d) Fusion image after registration

Fig. 3.9 Registration results of slice No.94 FDG_CT and FLT_CT image

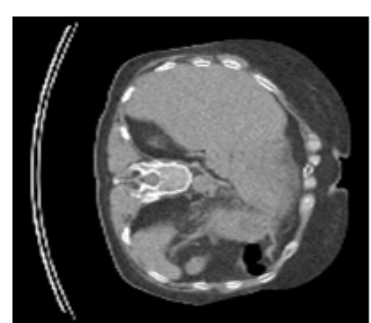

(a) Slice No. 95 FDG_CT

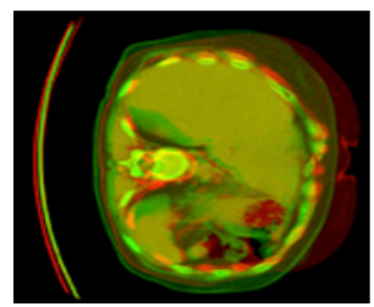

(c) Fusion image before registration

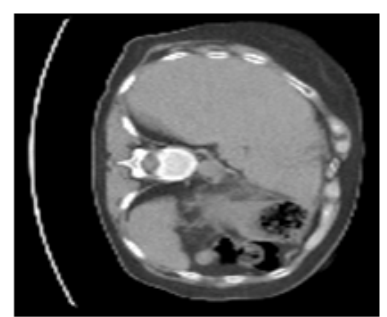

(b) Slice No.95 FLT_CT

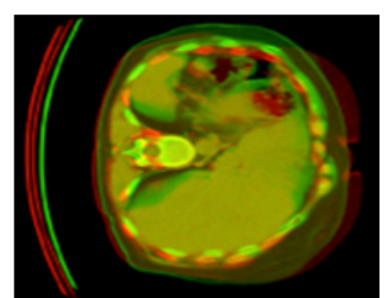

(d) Fusion image after registration

Fig. 3.10 Registration results of slice No.95 FDG_CT and FLT_CT image 


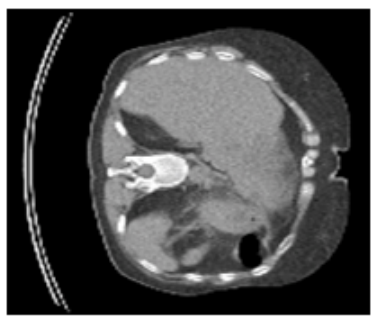

(a) Slice No.96 FDG_CT

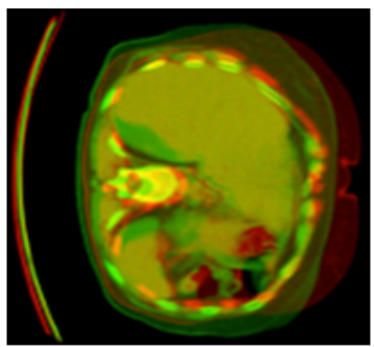

(c) Fusion image before registration

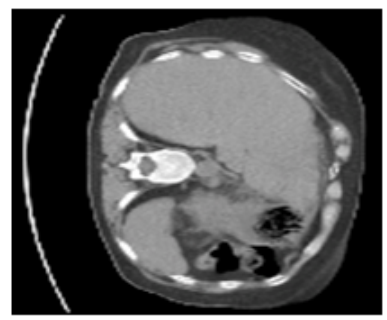

(b) Slice No.96 FLT_CT

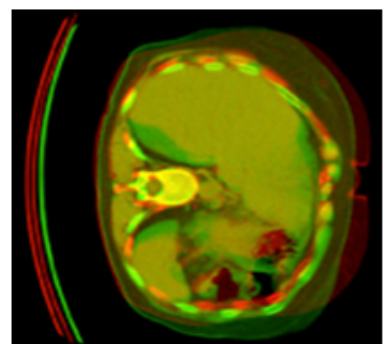

(d) Fusion image after registration

Fig. 3.11 Registration results of slice No.96 FDG_CT and FLT_CT image

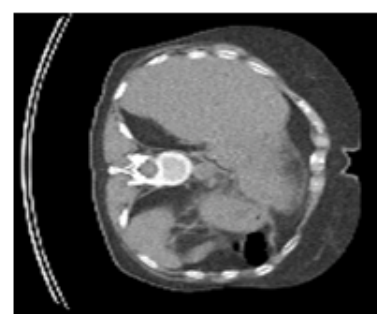

(a) Slice NO.97 FDG_CT

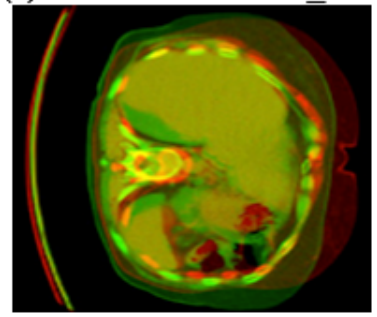

(c) Fusion image before registration

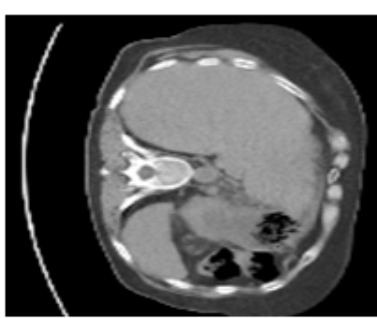

(b) Slice NO.97 FLT_CT

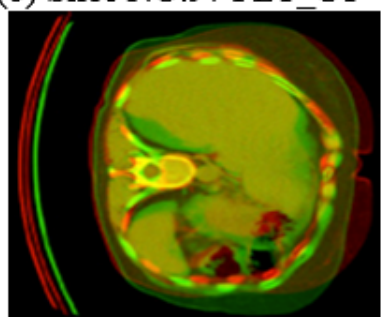

(d) Fusion image after registration

Fig. 3.12 Registration results of slice No.97 FDG_CT and FLT_CT image 


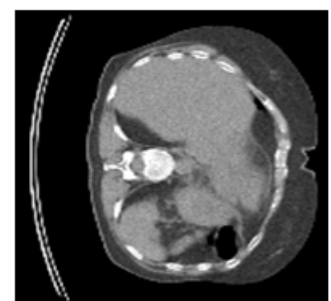

(a) Slice NO.98 FDG CT

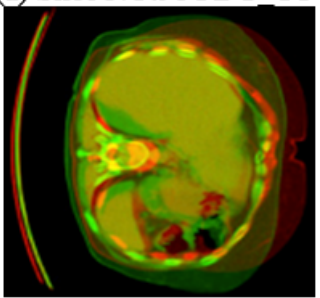

(c) Fusion image before registration

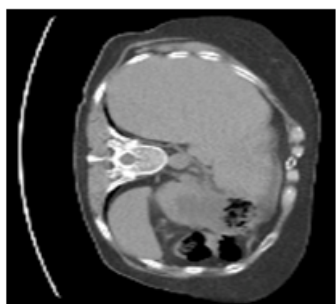

(b) Slice NO.98 FLT CT

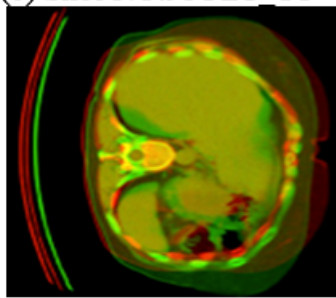

(d) Fusion image after registration

Fig. 3.13 Registration results of slice No.98 FDG_CT and FLT_CT image

The results are shown in Fig. 3.7 through Fig. 3.13 reveal the raw image of FDG_CT and FLT_CT before and after registration by GPW. In order to see the different parts of these two kinds of CT images, putting FDG_CT in the red channel, and the FLT_CT in the green channel, the fusion image of them should yield a yellowish color. The spine in the fusion images is not overlapping $100 \%$ before registration. But, after registration by GPW, the FDG_CT was put into the red channel, and the registered image was put into the green channel, then the spine turns to the color yellow. That meant that the spine is overlapping better than before. The cancerous lesions parts of the liver could be observed directly on the FLT_CT image in red color.

\subsection{Conclusion}

In this chapter, registration of seven pairs of FDG_CT and FLT_CT images using an integrated GPW approach is accomplished. Registration results were improved in terms 
of quality of the fused images in terms of NMI and visual observation, showing a good overlap of the two modalities, but also in terms of the significantly reduced computational requirements. From the fusion image, the location of the cancerous lesions on the liver, which were shown in red color, could be observed directly on the FLT_CT image. This outcome could help enhance both the delineation of tumors and cancer diagnosis. In retrospect, the GPW approach is shown to reduce the computational burden of GA when searching for the best global solution and prevents the PM in locking onto a best local solution for image registration, which may not be the optimal solution. The proposed method resolves both of these issues. 


\section{MULTI-MODALITY MEDICAL IMAGE REGISTRATION}

\subsection{Introduction}

Medical images have always been essential in the delivery of healthcare to patients who have to image for treatment planning. Within medical research, especially neuroscience research, they are used to investigate disease processes and understand normal development from the disease state. In many of these studies, multiple images are acquired from subjects at different times, and often with different imaging modalities. In such multimodal research studies, not only is it important in seeking good registration for the optimal fusion of the different modalities, but it is also desirable to compare images obtained from patient cohorts in support single subjects imaged multiple times.

This chapter describes a fully integrated multimodal approach to medical image registration. For its implementation, this chapter provides the data used in the registration experiments and defines the goals sought through these experiments. To assess the performance of this integrated method, a comparative study is performed to evaluate the results as obtained by the proposed method contrasted with the most established and highly successful software platforms of FSL and SPM.

\subsection{Data}

There are 4 sets of data in this study, including one whole-body CT image and the brainonly images. The details of the data are as follows: 
Dataset \#1 includes five patients with resectable and unresectable pancreatic cancers who underwent 18F-FLT and 18F-FDG imaging, each acquired within a week's duration for the same patient. FDG_CT is short for fluorine18-fluorodeoxyglucose. FLT_CT is short for fluorine18-fluorothymidine. In these 5 datasets that have been tested, it is noted that each of them had FLT_CT and FDG_CT taken in different days. FDG_CT, in this study, is used as the reference image, which is unmoved, while the FLT_CT is used as the input image, which is moving in seeking that perfect alignment. Except for data set 1 which was of size $(512 * 512 * 186)$, all other sets were of size $(512 * 512 * 244)$ for both FDG_CT and FLT_CT image modalities.

Dataset \#2 includes 5 patients with tumors in their brains who underwent pre-operation brain MRI and intra-operation brain CT within a week's duration for the same patient.

Dataset \#3 includes 5 patients with Alzheimer's disease. They underwent brain MRI and PET within few days to two months duration for the same patient.

Dataset \#4 includes 1 normal control's T1, T2, fluid-attenuated inversion recovery image (FLAIR), and Gradient and spin-echo images (GE). 


\subsection{Experiments}

\subsubsection{The Goal of the Four Experiments}

Experiment 1 compares the whole body registration results using data set \# 1 by the proposed method, the co-register function of SPM 12, and the FLIRT linear registration function of FSL, respectively.

Experiment 2 compares the multi-modalities registration results using data set \# 2 through registering MRI and CT images by the proposed method, the co-register function of SPM 12, and the FLIRT linear registration function of FSL, respectively.

Experiment 3 compares the multi-modalities registration results using data set \# 3 through registering MRI and PET images by the proposed method, the co-register function of SPM 12, and the FLIRT linear registration function of FSL, respectively.

Experiment 4 compares the same modality registration results using data set \# 4 through registering $\mathrm{T} 1$ and $\mathrm{T} 2, \mathrm{~T} 1$ and FLAIR, T1 and GE images by the proposed method, the co-register function of SPM 12, and the FLIRT linear registration function of FSL, respectively. 


\subsubsection{Procedures of the Proposed Method}

The following flowchart, as shown in Fig. 4.1, illustrates the essential steps that were implemented to image registration. The procedure of the proposed method is described in the following 7 steps:

1) Trilinear interpolation to unify dimensions of original reference and moving image

The images obtained from different modalities usually have different dimensions. In order to calculate their NMI, it is necessary to interpolate the moving image (Imov) to obtain the same dimension of the reference image (Iref). In this study, trilinear interpolation is employed to obtain the interpolated moving image.

2) NMI calculation to find the best matching pair

Three slices ( $i, j$, and $k$ ), whose anatomy characters are easily observed, have been manually selected from Iref ( $\mathrm{i}, \mathrm{j}$, and $\mathrm{k}$ are positive real numbers). Based on calculating the NMI, three slices (p, q, and r) will be selected form Imov. For example, the NMIs between slice $i$ and each of the slices of Imov have been calculated; then the largest value (MaxNMI) among the NMIs will be found; the index of the MaxNMI is p. Therefore, slice $\mathrm{p}$ of Imov is selected as the best matching for slice i of Iref, the same method for slice $q$ and $r$. Slice $i$ and $p$ is the first pair; slice $j$ and $q$ is the second pair; and slice $k$ and $r$ is the third pair ( $p, q$, and $r$, are positive real numbers). 
3) Wavelet decomposition to obtain the smaller, low-frequency part of the selected three pairs

The computational time is huge when using the Genetic Algorithm (GA) to optimize the registration results, especially for the medical images. Using smaller images instead of the original large ones could sharply reduce the consuming time. The wavelet decomposition method can offer the smaller size image, meanwhile, maintain most of the information of the original image [53]. Therefore, the wavelet decomposition method is applied to the selected three pairs of images to get the smaller, low-frequency images (Li, Lj, Lk, LP, Lq, and Lr).

\section{4) Initial Search by GA}

The Genetic Algorithm has been applied to each pair of the image, and three sets of registration parameters for the best matching pairs have been obtained. The initial searching parameters for Powell Method (PM) are set by the obtained parameters.

\section{5) Second Search by PM}

The registration results are re-optimized by Powell Method (PM). In this way, the registration is enhanced since PM will search around the initial point set found by GA. This second search will apply PM to the original large images (Iref and Imov).

\section{6) Linear Interpolation}

The three sets of registration parameters are segmented linear interpolated to get the other sets of parameters for all slices. The registration parameters from slice \# $\mathrm{i}$ to slice \# $\mathrm{j}$ can 
be achieved by linear interpolating the registration parameters between the first pair and the second pair. The parameters of slice \# 1 to slice \# (i-1) can be achieved by linear extend the parameters of slice \# $\mathrm{i}$. The registration parameters of slice \# $(\mathrm{j}+1)$ to slice \# $\mathrm{k}$ can be achieved by linear interpolating the registration parameters between the second pair and the third pair. The parameters of slice \# $(\mathrm{k}+1)$ to the end can be achieved by linear extend the parameters of slice \# $\mathrm{k}$. Therefore, the final solutions for all slices are obtained in this way.

7) Registration

Based on the final solution for all slices, Imov has been moved to align with Iref through Affine transformation [55 - 61]. 


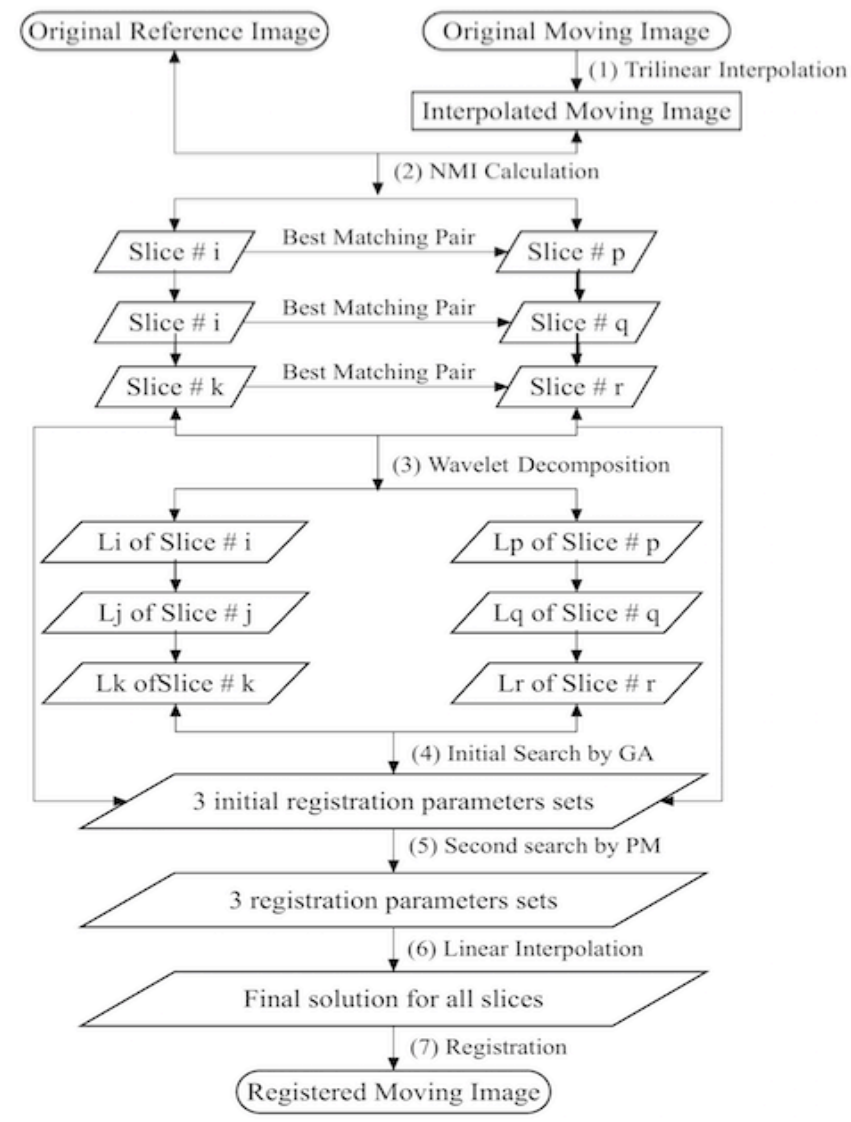

Fig. 4.1 Flowchart of the proposed method

\subsubsection{Evaluation Method}

In this chapter, the normalized mutual information will be calculated as the evaluation standard for image optimization. Unlike many other registration methods, mutual information makes fewer priority assumptions about the object, making it adaptable to changes in lighting and changes between sensors. It can be applied to larger dimensional medical image registration. In order to know the registration quality, normalized mutual 
information (NMI) is defined below. The value of NMI will be in the range of 0 to 1 . The closer to 1 , the better the registration is.

\subsection{Optimizing Methods}

In this study, between each pair of a reference image and moving image, there can be horizontal displacement, vertical displacement, scaling, and rotation. There is however no specific functional expression for expressing gradient change. Based on these situations, the Powell method (PM) could be a suitable optimization algorithm since it is good at finding the best solution where the calculation of the gradient is not possible. However, to apply PM here, the initial searching point must be close to the optimal solution. Therefore, the Genetic Algorithm (GA) is applied first to find the global optimal solution. Then, starting from that global optimal solution, PM will find the best solution for optimization of the final results. In addition, GA is time-consuming when applied to the original images. To save time, the wavelet decomposition will be used to reduce the dimension of the original images.

\subsubsection{Genetic Algorithm (GA)}

The essence of GA is Darwin's theory of evolution. The main idea is encoding the parameters of registration, as different genes, in a chromosome, and generating a population of potential solutions as the first generation randomly; then letting them evolve with respect to "the fittest survives" rule-selection, crossover, and mutation; finally, decoding the best chromosome in the last generation. In this study, the translation 
of $\mathrm{x}$-axis (x), the translation of y-axis (y), scaling (k), and rotation (angle) are the registration parameters. Based on the data, the same scaling parameter $\mathrm{k}$ has been used in both the $\mathrm{x}$-axis and $\mathrm{y}$-axis direction. Scaling and rotation are both from the image's center of gravity. One set of $\mathrm{x}, \mathrm{y}, \mathrm{k}$, and angle is a frame. The best registration resolution is the best frame, which gains the highest NMI. GA processing procedures are shown in the following Fig. 4.2.

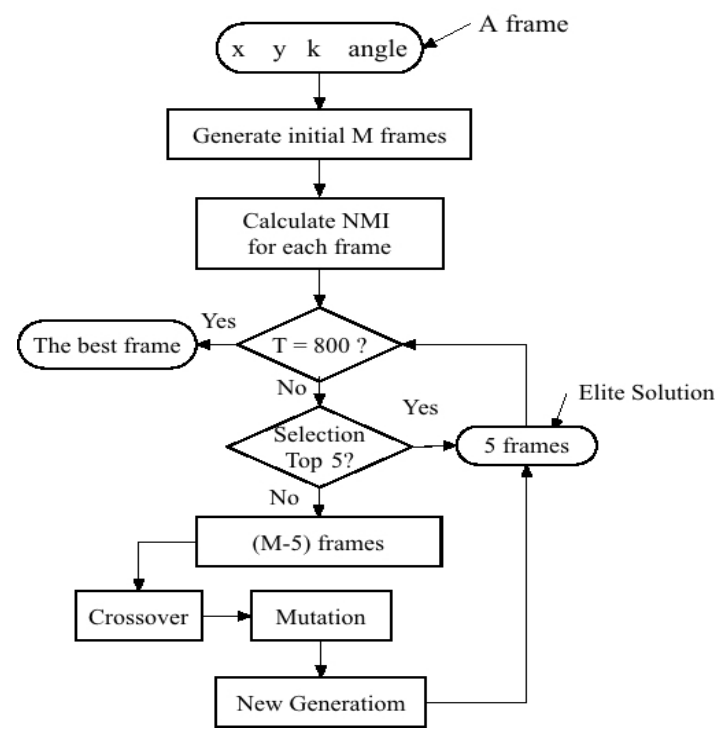

Fig. 4.2 Flowchart of the Genetic Algorithm

1) Generation of the initial $M$ frames

The population of the potential solution is $\mathrm{M}$. M frames will be generated at the beginning of GA. Each of the frame includes a set of $x, y, k$, and angle. Based on the data in this study, -10 to 10 will be enough for the translation, scaling, and rotation. Based on the experiment, when $\mathrm{M}=320$, GA gets the highest NMI. Therefore, 320 frames of $\mathrm{x}, \mathrm{y}$, 
$\mathrm{k}$, and angle will be generated randomly from $[-10,10]$. Those 320 frames form the first generation of GA.

2) NMI calculation for each frame

Calculating NMI values uses equations 1 to 5 . NMI is the evaluation parameter for each frame in each generation. In this study, the frames with top 5 highest NMI values, which are the elite solutions, will be directly copied into the next generation without Crossover and Mutation applied to them. The other 315 frames will go to Selection, Crossover, and Mutation.

\section{3) Selection}

This step will duplicate the frames depending on the Fitness Proportionate Selection (Roulette Wheel Selection) method [23, 24]. Each frame is corresponding to an NMI value. If $N M I_{i}$ is the NMI value of individual $i$ in the population $n$, its probability of being selected $\left(S P_{i}\right)$ is as follows:

$$
S P_{i}=\frac{N M I_{i}}{\sum_{j=1}^{n} N M I_{j}}
$$

After calculating the probability of being selected $\left(S P_{i}\right)$, the accumulate probability $\left(C P_{i}\right)$ will be calculated. Then, a number R1 will be randomly generated between $[0,1]$. R1 will work as a pointer. When it is close to a $C P_{i}$, the individual $\# \mathrm{i}$ will be selected. In this 
study, $\mathrm{n}=315$, and $\mathrm{i}=1,2, \ldots, 315$. The total number of $\mathrm{R} 1$ is 315 . Those $\mathrm{R} 1 \mathrm{~s}$ will point to 315 frames. Those frames will go to the Crossover and Mutation steps.

For example, Fitness Proportionate Selection method will select 5 frames from 10 frames. The NMI values $\left(N M I_{i}\right)$, the probability of being selected $\left(S P_{i}\right)$, and the accumulate probability $\left(C P_{i}\right)$ are shown in table 1:

Table 4.1 Example of Fitness Proportionate Selection

\begin{tabular}{|c|c|c|c|c|c|c|c|c|c|c|}
\hline Frame \# & 1 & 2 & 3 & 4 & 5 & 6 & 7 & 8 & 9 & 10 \\
\hline$N M I_{i}$ & 0.40 & 0.70 & 0.18 & 0.50 & 0.10 & 0.30 & 0.15 & 0.60 & 0.60 & 0.60 \\
\hline$S P_{i}$ & 0.05 & 0.09 & 0.23 & 0.06 & 0.13 & 0.04 & 0.19 & 0.08 & 0.08 & 0.08 \\
\hline$C P_{i}$ & 0.05 & 0.14 & 0.36 & 0.43 & 0.55 & 0.59 & 0.78 & 0.85 & 0.93 & 1.00 \\
\hline
\end{tabular}

The 5 numbers randomly selected are $0.94,0.25,0.31,0.66$, and 0.53 . According to table 4.1, the selected individual numbers are: 10, 3, 3, 7, and 5, as shown in Fig. 4.3.

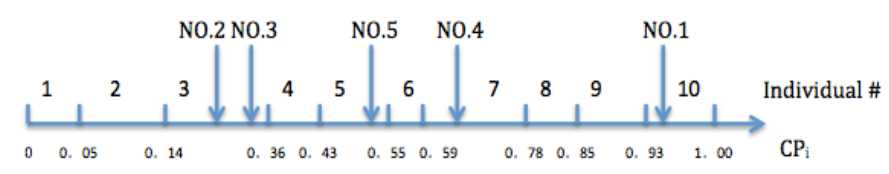

Fig. 4.3 Selection operation

\section{4) Crossover}

The crossover exchanges portions of the frame to produce better candidate frames with higher NMI values in the next generation $[51,52]$. According to a crossover probability $\left(P_{c}\right)$, the crossover point will be randomly determined, and then the portion starting from 
the crossover point will be exchanged. One-point-crossover is adopted in this study. Table 4.2 shows an example of that method. First of all, the crossover probability $P_{c}$ between the first frame and each of the odd-numbered frames will be calculated by equation 7. The even number frames will go to the Mutation directly. After that, the computer will generate a random number R2 from $[0,1]$. Then, each $P_{c}$ will compare to $\mathrm{R} 2$, if $P_{c}$ larger than or equal to $\mathrm{R} 2$, a crossover point will be determined randomly from the first frame, and the portions starting from the crossover point will be exchanged. If $P_{c}$ is less than R2, no crossover will take place here. The original frame will be kept to the next step. That comparison has been done 315 times to make 315 frames, which include the exchanged frames and the kept original frames. Those frames will go to the Mutation step.

Table 4.2 Example of One-point-crossover

\begin{tabular}{|c|c|c|c|c|c|c|c|c|c|c|}
\hline Father Frame 1 & 1 & 0 & 1 & 0 & 1 & 1 & 0 & 0 & 1 & 0 \\
\hline Father Frame 2 & 0 & 1 & 0 & 1 & 1 & 0 & 0 & 1 & 0 & 1 \\
\hline \multicolumn{11}{|c|}{ One-point-crossover } \\
\hline New Frame 1 & 1 & 0 & 1 & 1 & 1 & 0 & 0 & 1 & 0 & 1 \\
\hline New Frame 2 & 0 & 1 & 0 & 0 & 1 & 1 & 0 & 0 & 1 & 0 \\
\hline
\end{tabular}

$f_{\text {avg }}$ is the average NMI value. $f_{\max }$ is the maximum NMI value. $f^{\prime}$ is the biggest NMI value between two frames in the father generation. The crossover probability $\left(P_{c}\right)$ can be defined as the following [23, 24]: 


$$
p_{c}=\left\{\begin{array}{cc}
P_{c 1}-\frac{\left(P_{c 1}-P_{c 2}\right)\left(f^{\prime}-f_{\text {avg }}\right)}{f_{\max }-f_{\text {avg }}} & f^{\prime} \geq f_{\text {avg }} \\
P_{c 1} & f^{\prime}<f_{\text {avg }}
\end{array}\right.
$$

The most common values in applications are: $P_{c 1}=0.9, P_{c 2}=0.6$, which are also used in this study.

\section{5) Mutation}

Mutation is the second way to produce new frames in GA. It randomly modifies some bits of the frames and is responsible for the research space exploration [51, 52]. It prevents the algorithm from being trapped in the local optimal value. Mutation probability $\left(P_{m}\right)$ is supposed to be small. In one generation, $f_{a v g}$ is the average value of NMI. $f_{\max }$ is the maximum value of NMI. $f$ is the NMI value of the frame before mutation. The mutation probability $\left(P_{m}\right)$ can be defined as $[23,24]$

$$
p_{m}=\left\{\begin{array}{cc}
P_{m 1}-\frac{\left(P_{m 1}-P_{m 2}\right)\left(f_{\max }-f\right)}{f_{\max }-f_{\text {avg }}} & f \geq f_{\text {avg }} \\
P_{m 1} & f<f_{\text {avg }}
\end{array}\right.
$$

The most common values in applications are: $P_{m 1}=0.1, P_{m 2}=0.001$, which are also used in this study. First of all, the mutation probability $P_{m}$ of each frame comes from the Crossover and will be calculated by equation 8 . After that, the computer will generate a random number R3 from $[0,1]$. Then, each $P_{m}$ will compare to R3, if $P_{m}$ larger than or equal to R3, randomly choose one point from the frame. If the value of that point is 0 , it will be changed to 1 ; if the value of that point is 1 , it will be changed into 0 . If $P_{m}$ less 
than R3, no Mutation will take place in this frame, it will be kept to the next step. That comparison has been done 315 times to make 315 frames which including the exchanged frames and the kept original frames. Those 315 frames and the elite solutions ( 5 frames) form the new generation.

6) Termination criteria

There are many termination criteria, such as finding an acceptable approximate solution, reaching a specific number of generation, the highest NMI value reaching a specific number in a generation, or the average NMI value reaching a specific number in a generation [48, 49]. In this study, considering the computational time, reaching 800 generation is the termination criteria. The frames will go through the Selection, Crossover, and Mutation 800 times, and then end with 320 frames. The frame with maximum NMI value will be the optimal solution.

\subsubsection{Powell Method (PM)}

From the initial point, which is the best solution found by GA, the cost function is optimized along a direction using a one-dimensional search method, Golden Section Search [62] in this study, and then continues to the next direction. Therefore, after a number of one-dimensional searches, PM will get extreme points. In this study, the initial point could be the best solution, but PM will search close to this point to verify if it is the best or the PM will find the best of the best in the local area. The best solution has the highest NMI value. Therefore, PM will calculate the NMI for each solution along the 
directions to find the maximum NMI value. PM processing procedures are shown in the following Fig. 4.4.

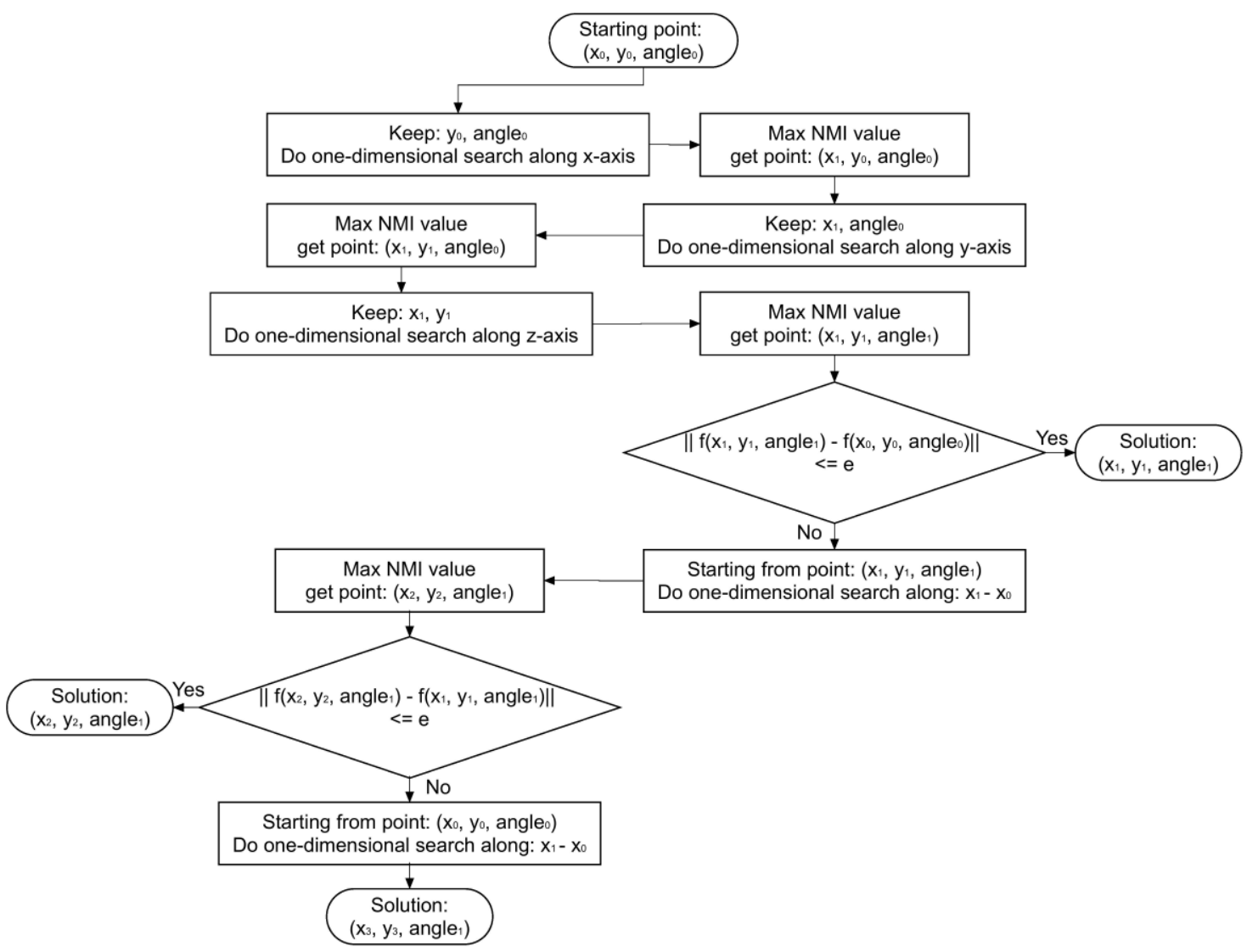

Fig. 4.4 Flowchart of PM

According to Fig. 4.4, GA gives its best frame for initial PM. PM will start its search from point $\left(\mathrm{x}_{0}, \mathrm{y}_{0}\right.$, angle $\left.{ }_{0}\right)$. First, $\mathrm{y}_{0}$ and angle $\mathrm{e}_{0}$ are unchanged, and $\mathrm{x}_{0}$ is moving along the $\mathrm{x}$-axis till finding the point $\left(\mathrm{x}_{1}, \mathrm{y}_{0}\right.$, angle $\left.\mathrm{e}_{0}\right)$ with the maximum value of NMI. Then, $\mathrm{x}_{1}$ and angle $e_{0}$ are unchanged, and $\mathrm{y}_{0}$ is moving along the $\mathrm{y}$-axis till finding the point $\left(\mathrm{x}_{1}, \mathrm{y}_{1}\right.$, angle ${ }_{0}$ ) with the maximum value of NMI. After that, $\mathrm{x}_{1}$ and $\mathrm{y}_{1}$ are unchanged, and angle $\mathrm{e}_{0}$ is moving along the $\mathrm{z}$-axis till finding the point $\left(\mathrm{x}_{1}, \mathrm{y}_{1}\right.$, angle $\left.{ }_{1}\right)$ with the maximum value 
of NMI. If the distance between $\left(\mathrm{x}_{1}, \mathrm{y}_{1}\right.$, angle $\left.\mathrm{e}_{1}\right)$ and $\left(\mathrm{x}_{0}, \mathrm{y}_{0}\right.$, angle $\left.\mathrm{e}_{0}\right)$ is less than or equal to $\mathrm{e}\left(\mathrm{e}=0.0001\right.$ in this study), the solution will be $\left.\left(\mathrm{x}_{1}, \mathrm{y}_{1} \text {, angle }\right)_{1}\right)$; if that distance larger than e, PM will do one more one-dimension search starting from the point $\left(\mathrm{x}_{1}, \mathrm{y}_{1}\right.$, angle $\left.{ }_{1}\right)$ along the direction of $\left(\mathrm{x}_{1}-\mathrm{x}_{0}\right)$ till finding the point $\left.\left(\mathrm{x}_{2}, \mathrm{y}_{2} \text {, angle }\right)_{1}\right)$ with the maximum value of NMI. If the distance between $\left(\mathrm{x}_{1}, \mathrm{y}_{1}\right.$, angle $\left.\mathrm{e}_{1}\right)$ and $\left.\left(\mathrm{x}_{2}, \mathrm{y}_{2} \text {, angle }\right)_{1}\right)$ is less than or equal to e, the solution will be $\left.\left(\mathrm{x}_{2}, \mathrm{y}_{2} \text {, angle }\right)_{1}\right)$; if that distance larger than e, PM will do another one-dimension search starting from the point $\left(\mathrm{x}_{0}, \mathrm{y}_{0}\right.$, angle $\left.\mathrm{e}_{0}\right)$ along the direction of $\left(\mathrm{x}_{1}-\mathrm{x}_{0}\right)$ till finding the point $\left.\left(\mathrm{x}_{3}, \mathrm{y}_{3} \text {, angle }\right)_{1}\right)$ with the maximum value of NMI. That point will be the solution.

\subsubsection{Wavelet Decomposition}

The Haar wavelet transform is applied, as shown in Fig.4.5, to decompose an original image into four sub-bands, including a low-frequency component L1 and three highfrequency components $(\mathrm{H} 1, \mathrm{D} 1, \mathrm{~V} 1)$. Then, $\mathrm{L} 1$ is processed by the second level wavelet transformation. L1 is decomposed into a second level low-frequency component L2 and three-second level high-frequency components (H2, D2, V2).

\begin{tabular}{|r|r|c|}
\hline \multicolumn{2}{|c|}{ H1 } & \multirow{2}{*}{ D1 } \\
\cline { 1 - 1 } H2 & D2 & \multirow{2}{*}{ V1 } \\
L2 & V2 & \\
\hline
\end{tabular}

Fig. 4.5 Wavelet Decomposition to the second level 
The high-frequency components are similar in the same direction but in different scales. The high-frequency components in the lower-resolution frequency band are similar to the high-frequency components in the higher-resolution frequency band. That means $\mathrm{H} 2$ is similar to H1, V2 is similar to V1, and D2 is similar to D1. The same with the lowfrequency component, L2 is similar to L1. [63 - 66] For example, the slice NO.93 of the FDG_CT image of dataset \# 1 is applied the wavelet decomposition to the second level. The size of this slice is 512-by-512. The original slice \# 93, the low- frequency and highfrequency parts of the first level and the second level are as shown in Fig. 4.6 to Fig. 4.8. In this study, the second level low-frequency part is used in the Genetic Algorithm.

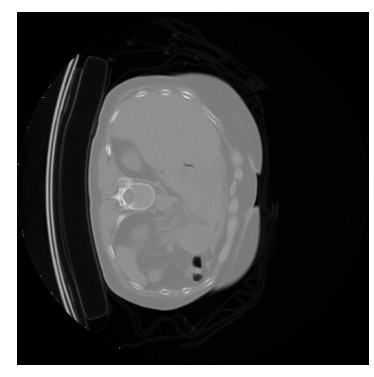

Fig. 4.6 Original slice \# 93 of FDG_CT image, image size: 512-by-512 


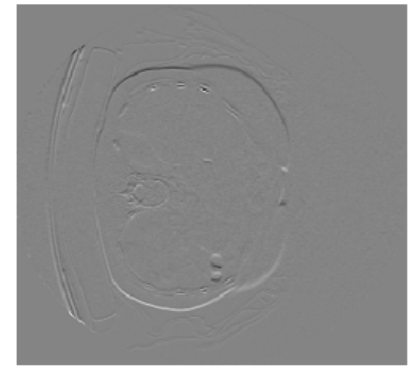

(a) $\mathrm{H} 1$

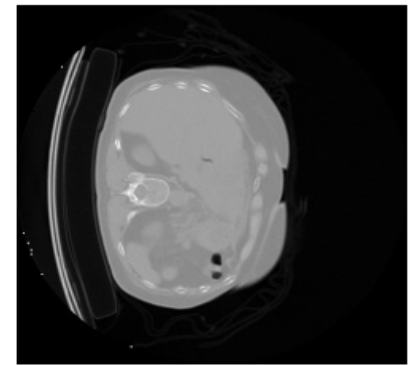

(c) L1

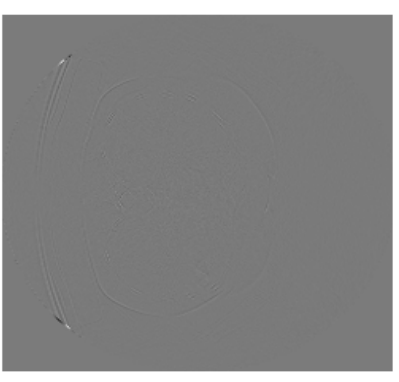

(b) D1

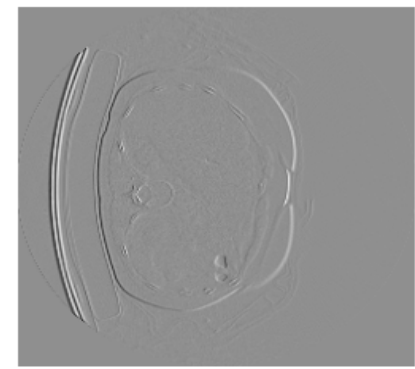

(d) V1

Fig. 4.7 Wavelet decomposition to the first level, image size: 256-by-256, low-frequency component: L1, and high-frequency components: H1, D1, and V1

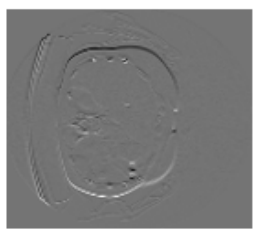

(a) $\mathrm{H} 2$

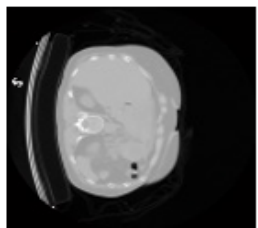

(c) L2

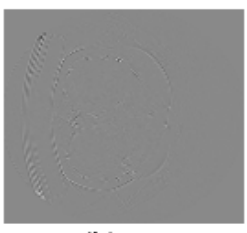

(b) D2

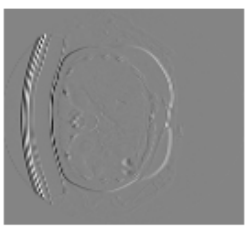

(d) V2

Fig. 4.8 Wavelet decomposition to the second level, image size: 128-by-128, lowfrequency component: L2, and high-frequency components: H2, D2, and V2 
The Genetic Algorithm (GA) needs a lot of processing time. To reduce that time, the smaller image, like (c) in Fig. 4.8, is used instead of the original large image in Fig. 4.6. In this way, the processing time will decrease significantly, as shown in Table 4.3.

Table 4.3 Processing times of wavelet decomposition, GA and the total

\begin{tabular}{|l|c|c|c|}
\hline Image Size & $\mathrm{T}_{\text {wavelet (sec) }}$ & $\mathrm{T}_{\mathrm{GA}(\mathrm{sec})}$ & $\mathrm{T}_{\text {total (sec) }}$ \\
\hline 512-by-512 & 0 & 3833 & 3833 \\
\hline 256-by-256 & 0.033 & 960 & 960.03 \\
\hline 128 -by-128 & 0.037 & 244 & 244.04 \\
\hline
\end{tabular}

According to Table 4.3, $\mathrm{T}_{\text {wavelet }}$ is the processing time of applying wavelet decomposition to the 512-by-512 images once and it takes $0.033 \mathrm{sec}$ and twice takes $0.037 \mathrm{sec} . \mathrm{T}_{\mathrm{GA}}$ is the processing time of GA when the application images are in the three different sizes. $\mathrm{T}_{\text {total }}$ is the total processing time. When using the smallest 128 -by-128 image, the GA only needs 244 secs, and including the wavelet decomposition time $0.037 \mathrm{sec}$, the total is 244.04 secs, which is far less than using the original 512-by-512 image. In this way, 3589 seconds have been reduced each slice. 6 slices from Dataset \#1 have been applied this method, and 2.99 hours have been saved in total. 


\subsection{Results and Analysis}

In this study, both of the inter-modality and multi-modality images have been registered by the proposed method. In order to compare the results, the co-register function of SPM 12 and the FLIRT linear registration function of FSL have been employed, too. For observation convenience, the reference images, which won't move, always appear in red; the moving images, which will be aligned to the reference image, always appear in blue; and the registered images always appear in green. The transverse, coronal, and sagittal images of the studying subjects will be displayed at the same time.

\subsubsection{Registration Results of Experiment 1-FDG_CT and FLT_CT}

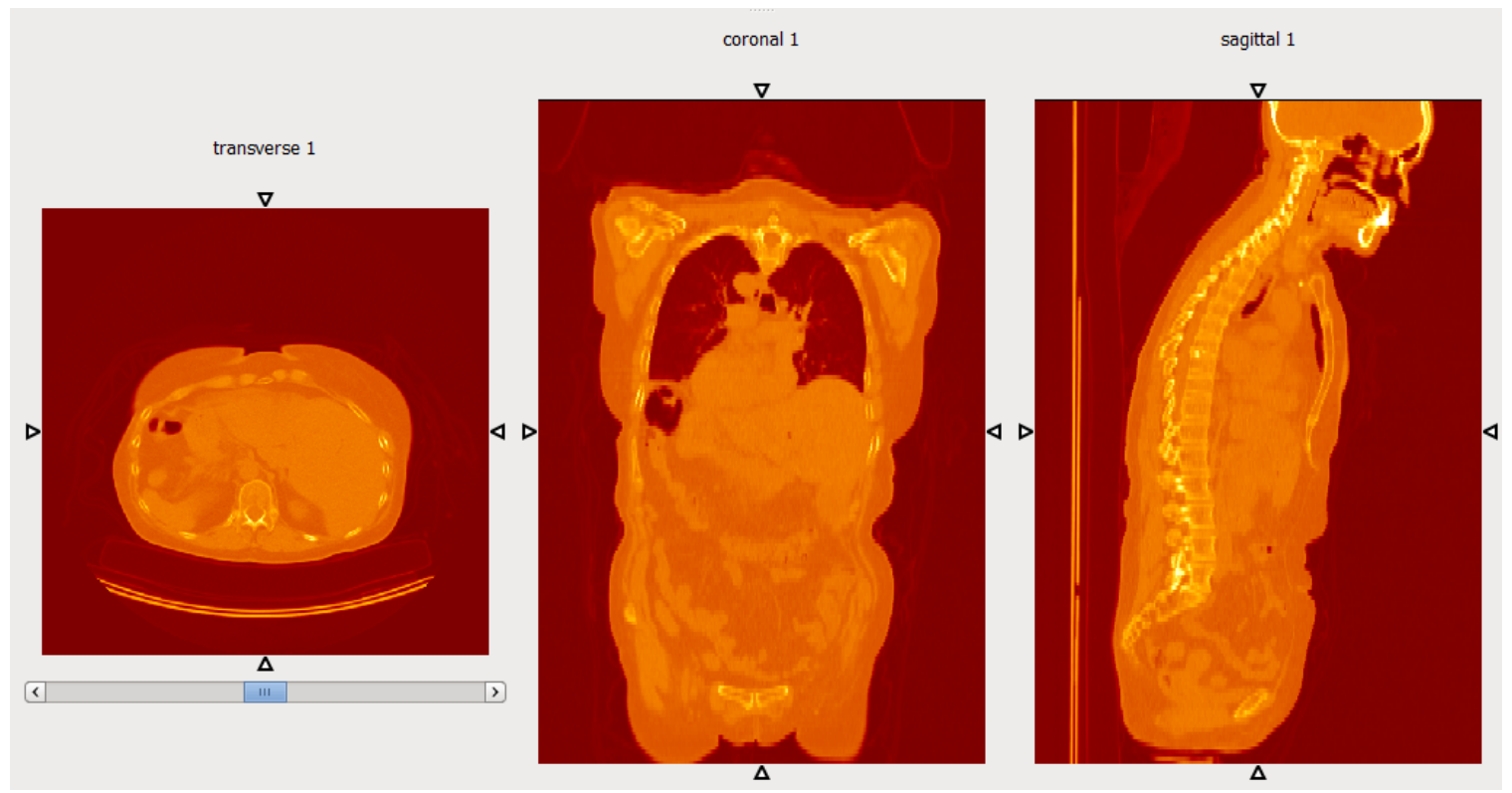

(a) FDG_CT 


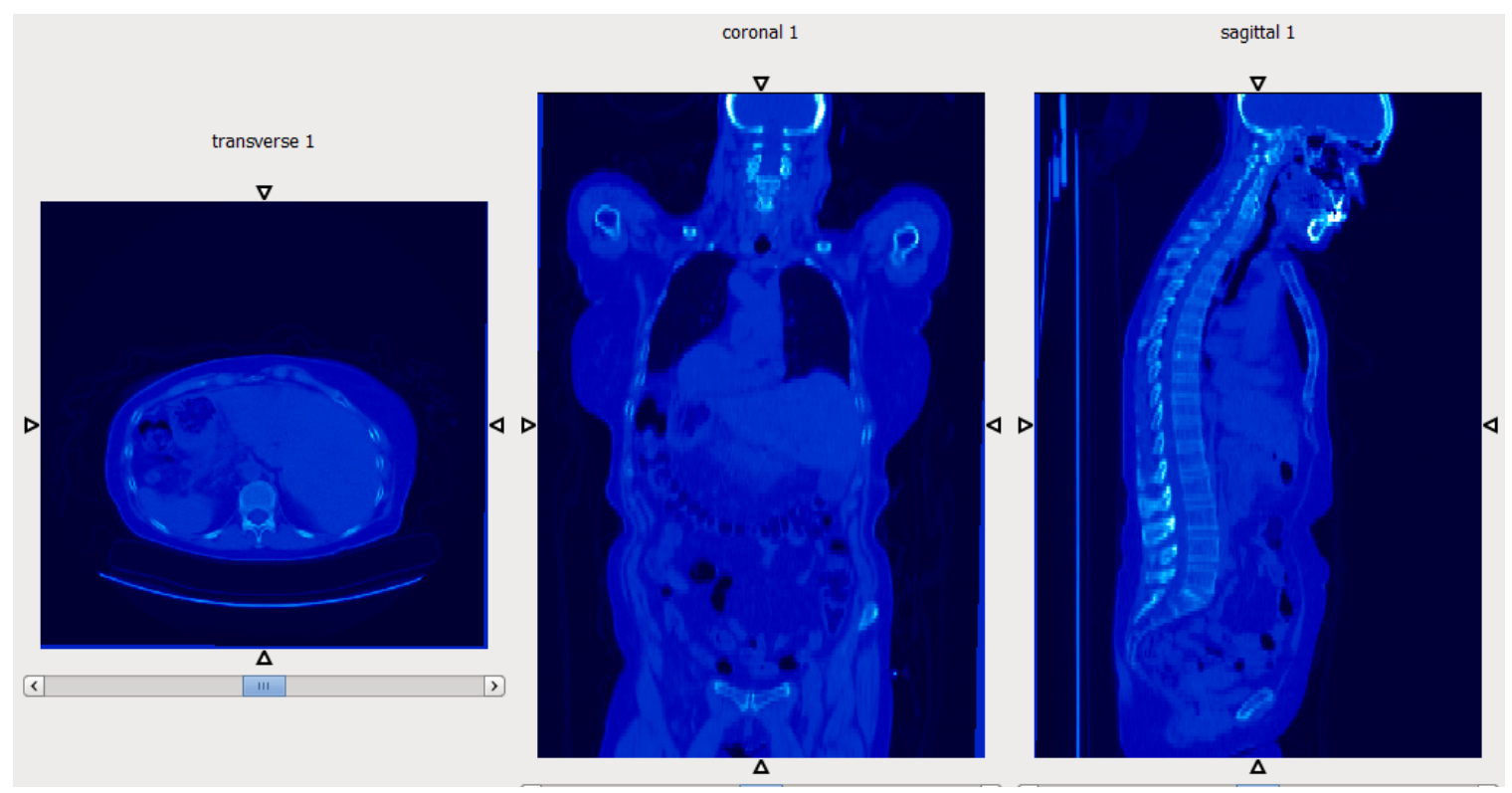

(b) FLT_CT

Fig. 4.9 Original images

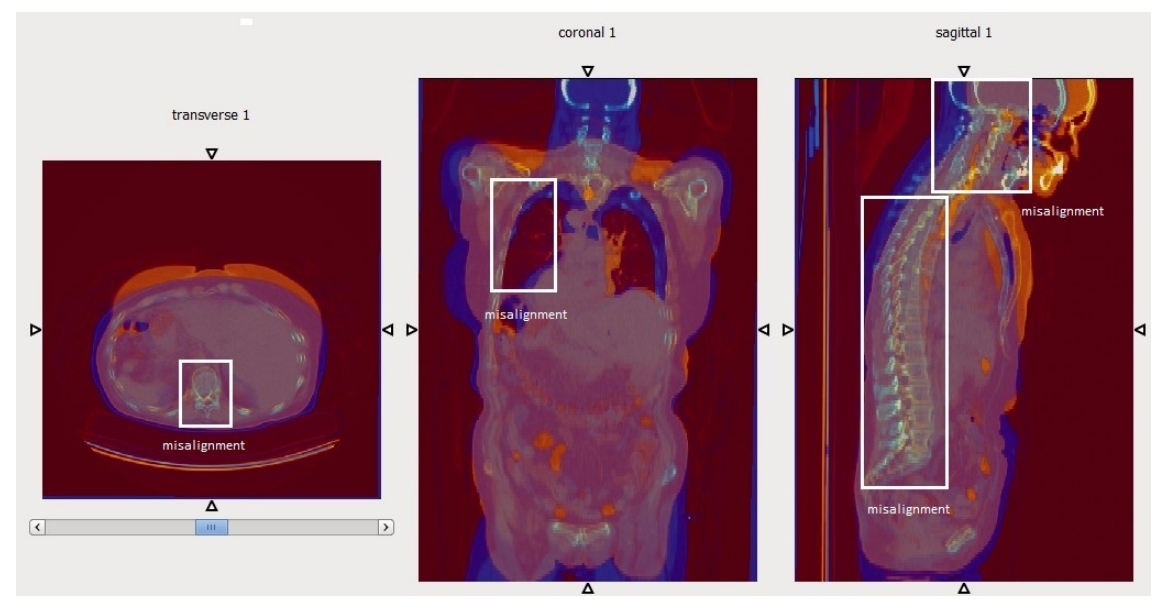

(a) Overlap of the original images 


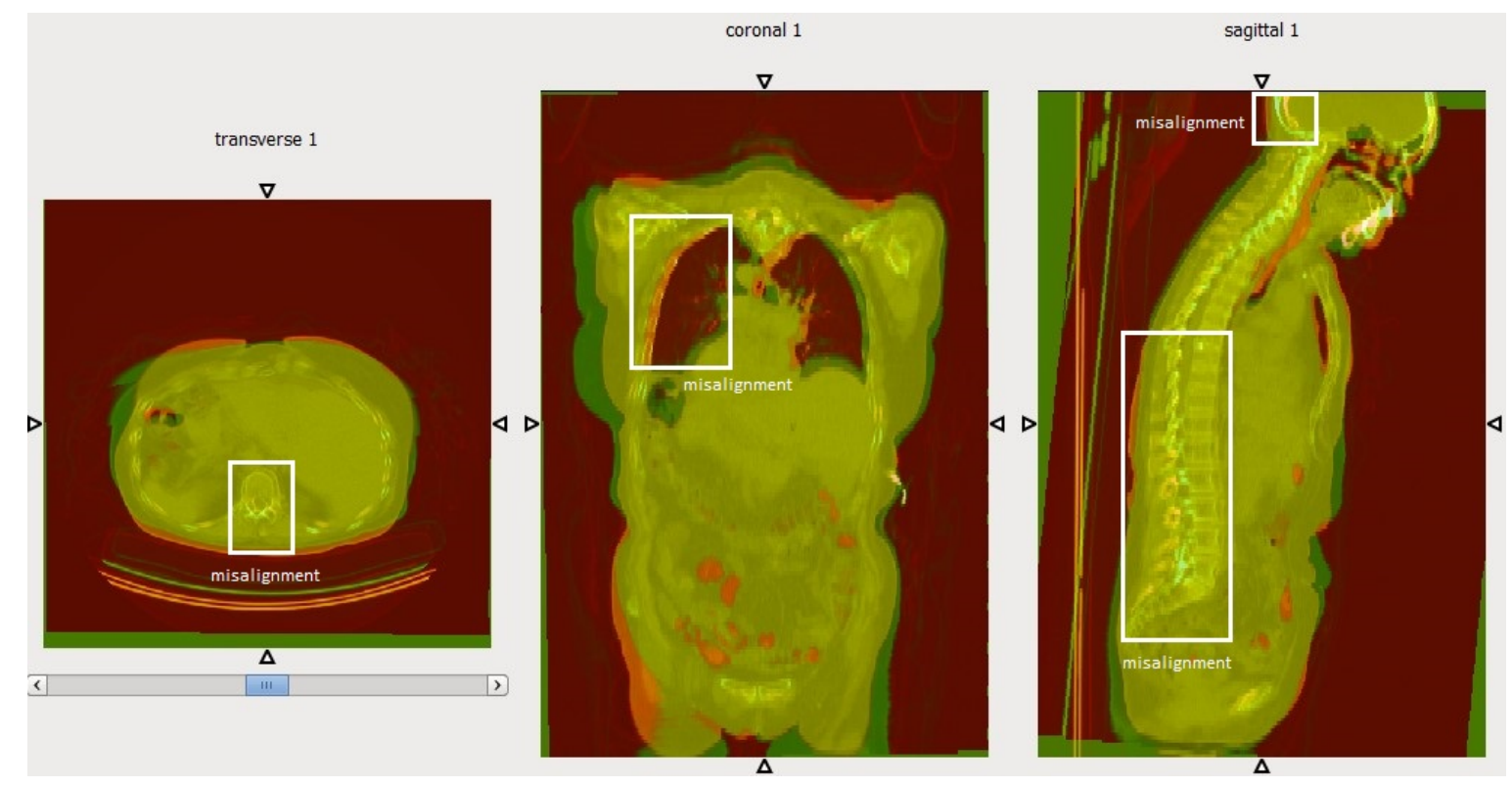

(b) SPM

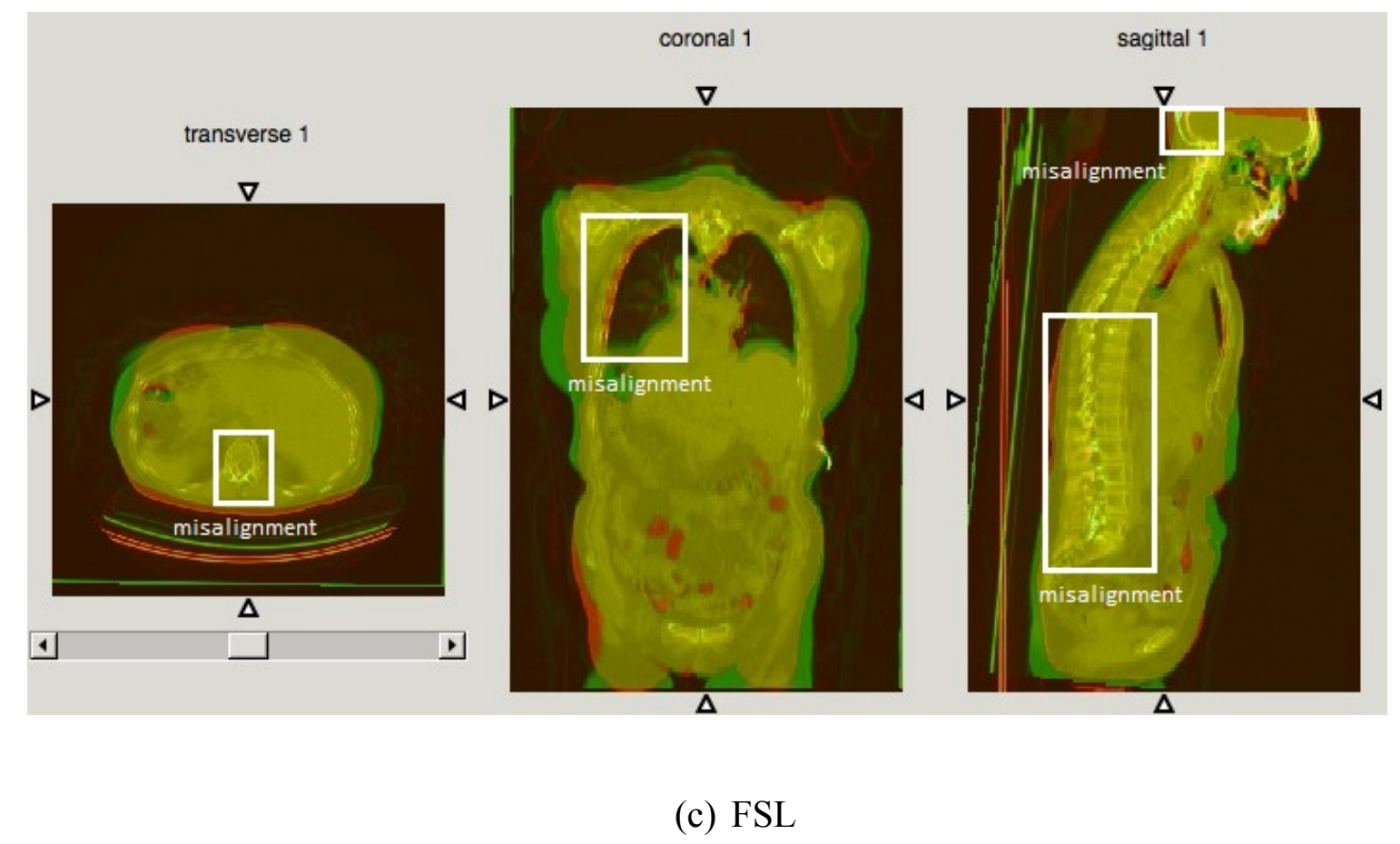




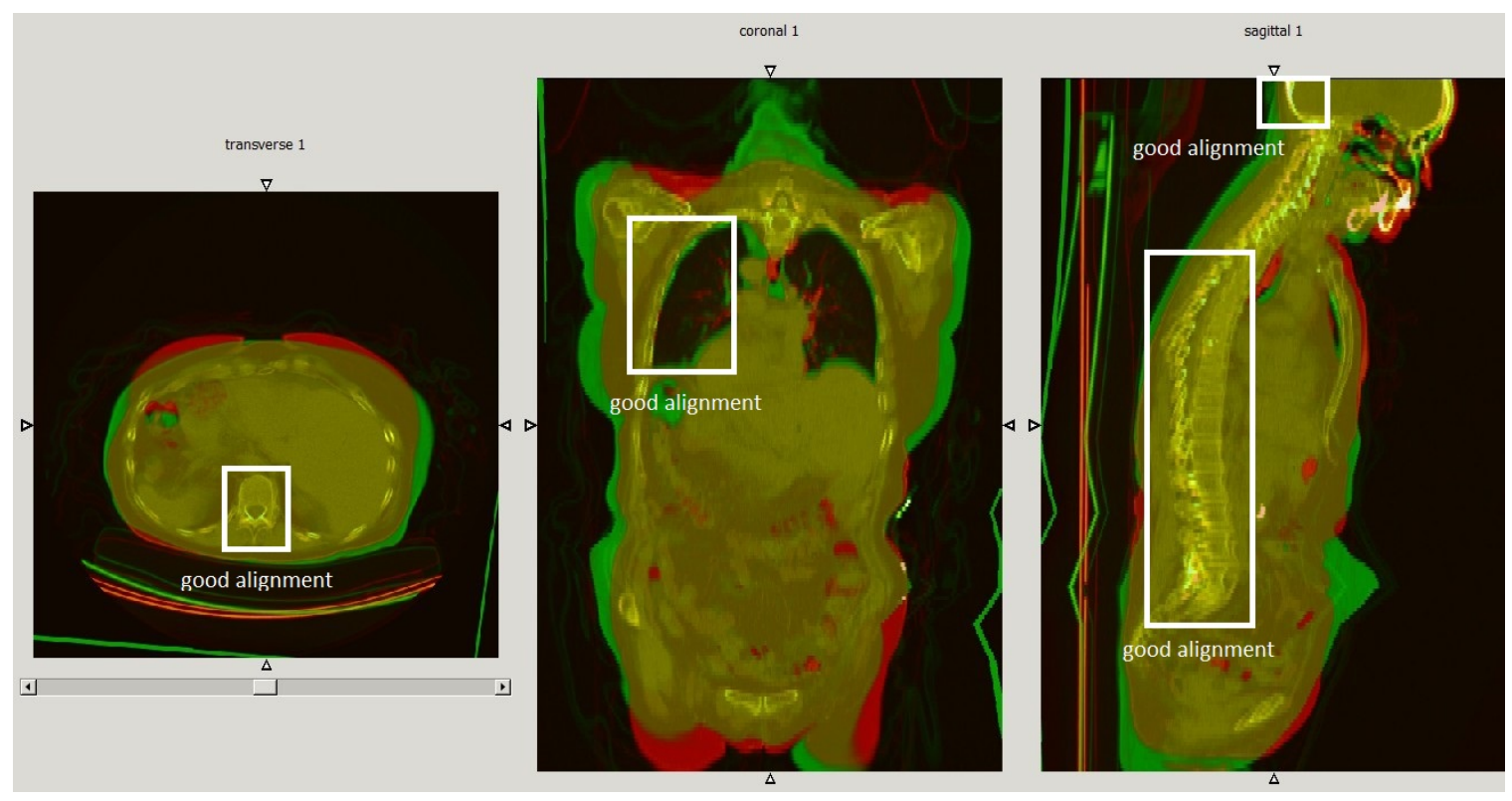

(d) The proposed method

Fig.4.10 Patient \# 1 of data set \#1, (a) Original FDG_CT (appears in red) and FLT_CT (appears in blue) are overlapped; (b) Registration results using SPM12; (c) Registration results using FSL; and (d) Registration results using the proposed method.

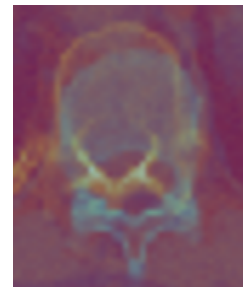

(a) Original

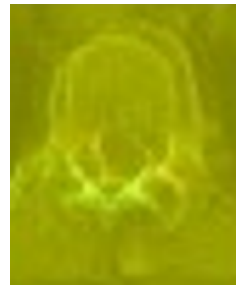

(b) SPM

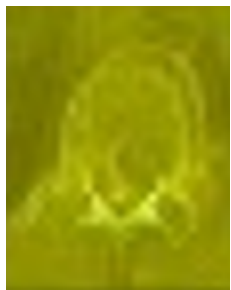

(c) FSL

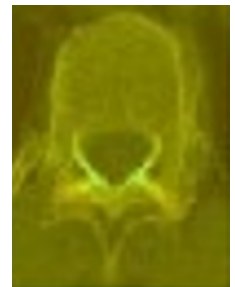

(d) Proposed method

Fig. 4.11 Enlarged spine in transverse image

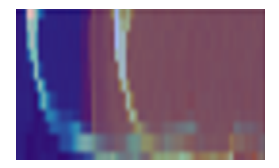

(a) Original

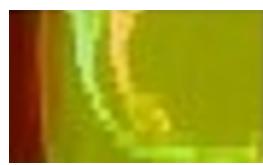

(b) SPM

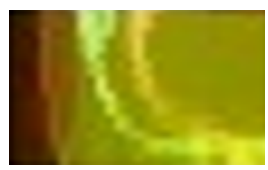

(c) FSL

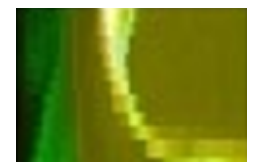

(d)Proposed method

Fig. 4.12 Enlarged skull in sagittal image 


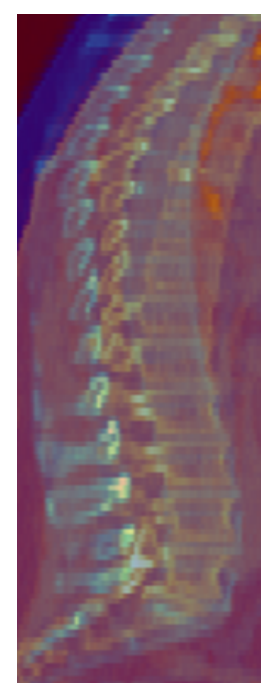

(a) Original

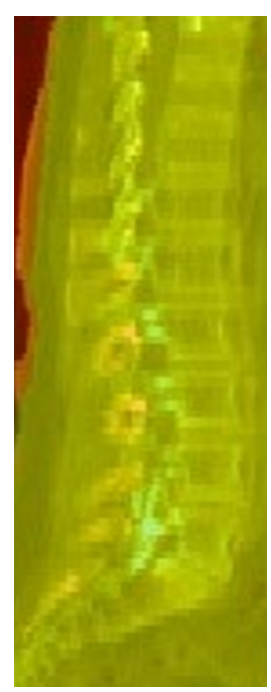

(b) SPM

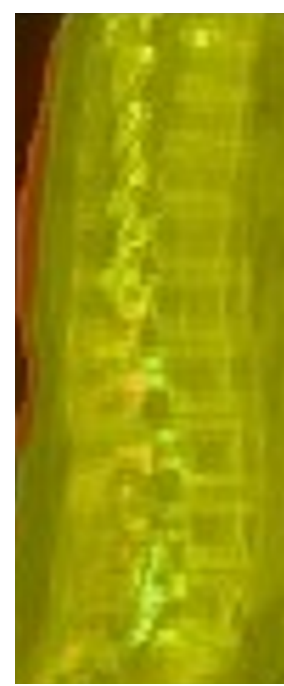

(c) FSL

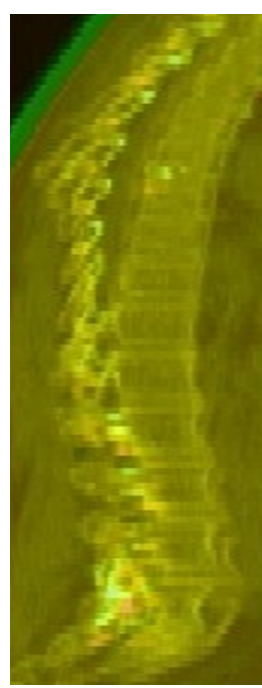

(d) Proposed method

Fig. 4.13 Enlarged spine and rib in sagittal image

In Fig. 4.9, the original FDG_CT is in red color, and the original FLT_CT image is in blue color. Fig. 4.10 is the registration result of the whole body CT image. The misalignment parts are shown inside the rectangle. Image (a) shows the overlapping of the original images. Image (b) and (c) show overlapping the registered image and the original FDG_CT by SPM and FSL, respectively. Although the misalignment part looks better than the corresponding part of the original images in (a), the misalignment includes: the spinal column in the transverse image, the rib and neck in the coronal image, and the brain and the spinal column in the sagittal image are still observed. Image (d), the proposed method corrects the misalignment effectively in the spinal column in the transverse image, the neck and rib in the coronal image, and the brain and spinal column in the sagittal image. Therefore, our proposed method shows the best function for registering the whole body CT image compare to SPM and FSL. For visualization purposes, Fig. 4.11 to 4.13 shows the enlarged spine in the transverse image, skull in the sagittal image, and spine and rib in the sagittal image. Both SPM and FSL, in (b) and (c), 
didn't correct all the misalignments, however, the proposed method shows perfect alignment in (d).

Table 4.4 Processing time and NMI comparison for the 5 data sets of experiment 1

\begin{tabular}{|c|c|c|c|c|}
\hline Data set \# & Evaluation parameter & SPM & FSL & Proposed method \\
\hline \multirow{2}{*}{$\# 1$} & T (s) & 566 & 960 & 610 \\
\cline { 2 - 5 } & Average NMI & 0.1989 & 0.2011 & 0.5361 \\
\hline \multirow{2}{*}{$\# 2$} & T (s) & 299 & 1500 & 706 \\
\cline { 2 - 5 } & Average NMI & 0.2040 & 0.2041 & 0.5543 \\
\hline \multirow{2}{*}{$\# 3$} & T (s) & 365 & 1321 & 945 \\
\cline { 2 - 5 } & Average NMI & 0.1991 & 0.2051 & 0.5311 \\
\hline \multirow{2}{*}{$\# 4$} & T (s) & 356 & 1446 & 928 \\
\cline { 2 - 5 } & Average NMI & 0.2011 & 0.1986 & 0.4776 \\
\hline \multirow{2}{*}{$\# 5$} & T(s) & 358 & 1357 & 518 \\
\cline { 2 - 5 } & Average NMI & 0.2026 & 0.2311 & 0.4276 \\
\hline
\end{tabular}

In Table 4.4, $\mathrm{T}$ is the processing time of registration process, and the average NMI is the average value of normalized mutual information between each of the registered images and the original reference image. Although the processing time is larger than SPM and less than FSL, our proposed method gained the highest average NMI value.

\subsubsection{Registration Results of Experiment 2-Pre-MRI and Intra-CT}

Fig. 4.14 is the original pre-operation MRI with the Intra-operation CT images. Fig. 4.15 is the registration result. The misalignment parts are shown inside the rectangle in the image. Image (a) is overlapping the original images. Image (b) is the registration results of SPM. Image (c) is the registration results of FSL. The misalignment in the image (a) is still in the image (b) and (c). SPM and FSL didn't register those kinds of the image well here. Image $(\mathrm{d})$ is the registration results of the proposed method. It works very well here and corrects most of the misalignment in image (a). Therefore, the proposed method is 
the best when registering the pre-operation MRI and intro-operation CT image compares to SPM and FSL. Fig. 4.16 and 4.17 show the skull in the coronal and the sagittal images. Both of the MRI and the CT skull can be visualized in the original image, the registered image by SPM or FSL. However, the proposed method only shows one skull. That means this registration method works perfectly when registering MRI and CT.

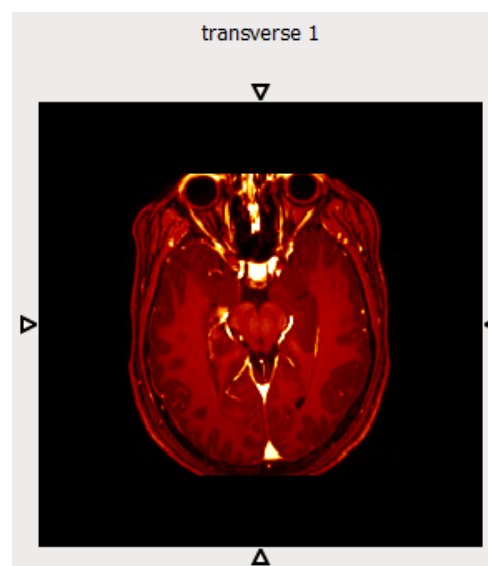

$\Delta$

transverse 1

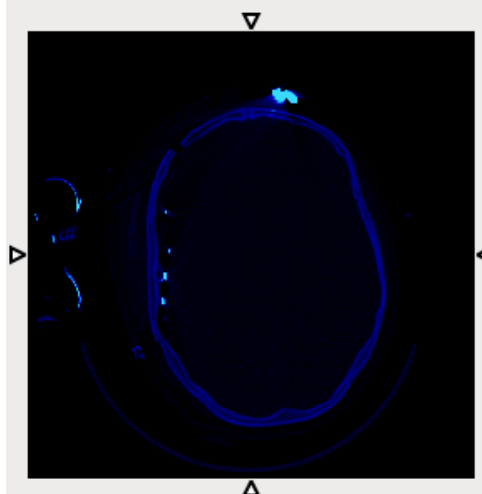

$\Delta$ coronal 1

$\nabla$

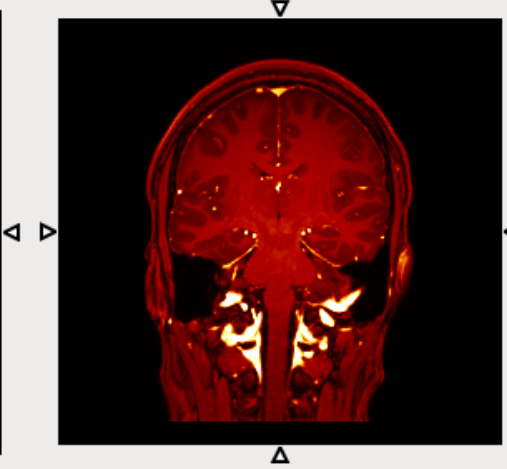

(a) Pre-operation MRI T1

coronal 1

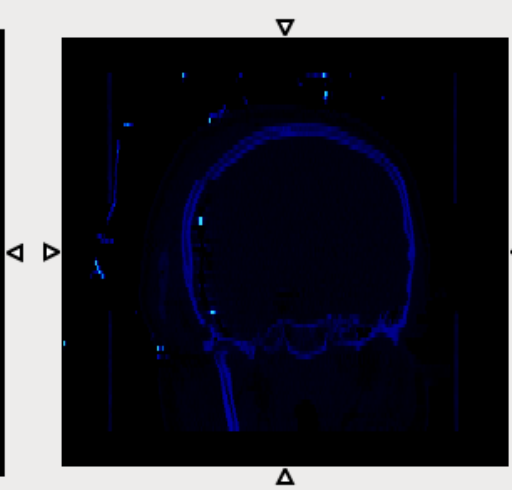

(b) Intra-operation CT sagittal 1

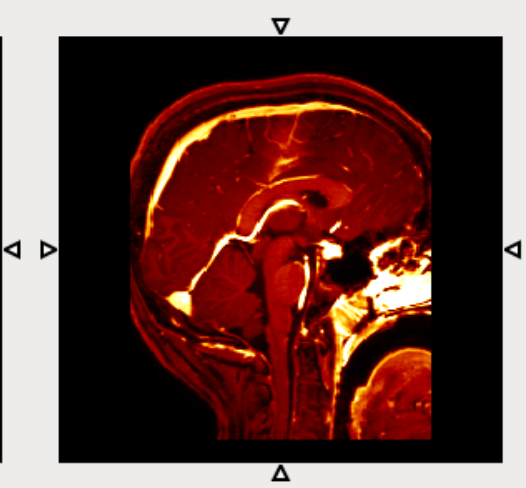

sagittal 1

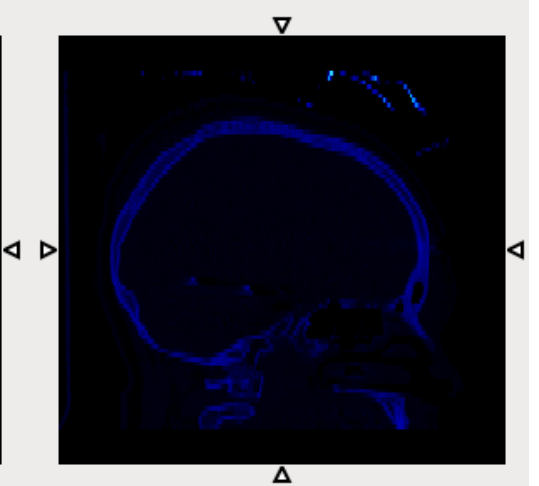

$\Delta$

Fig. 4.14 Original images 


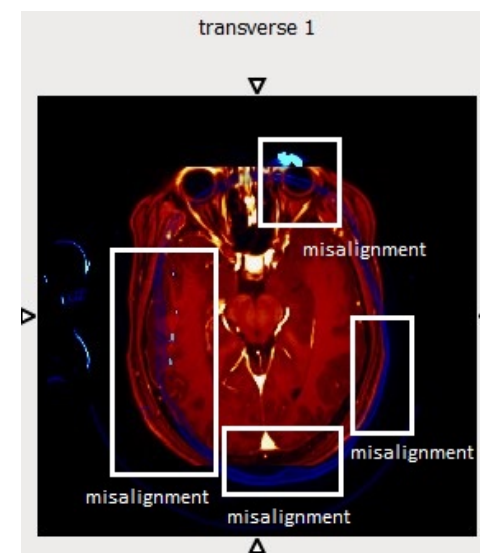

transverse 1
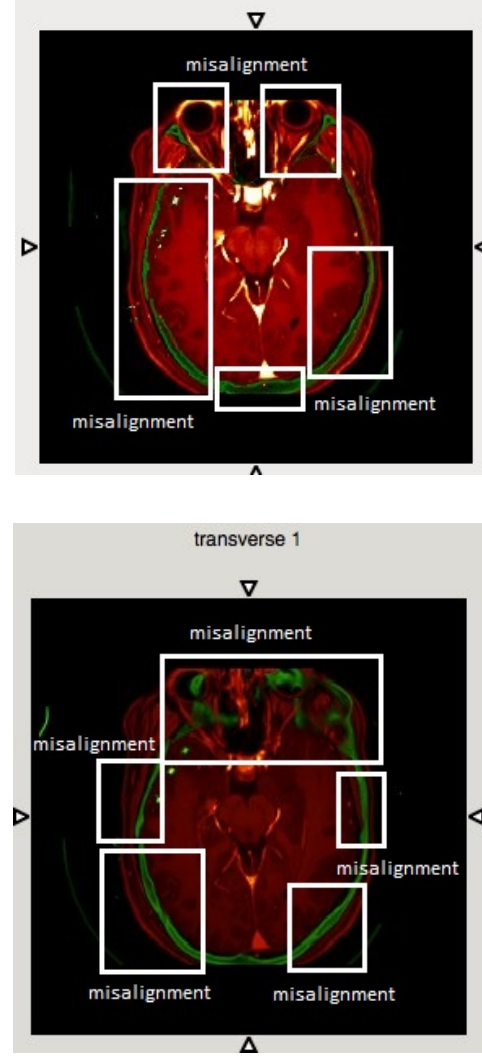

$\Delta$

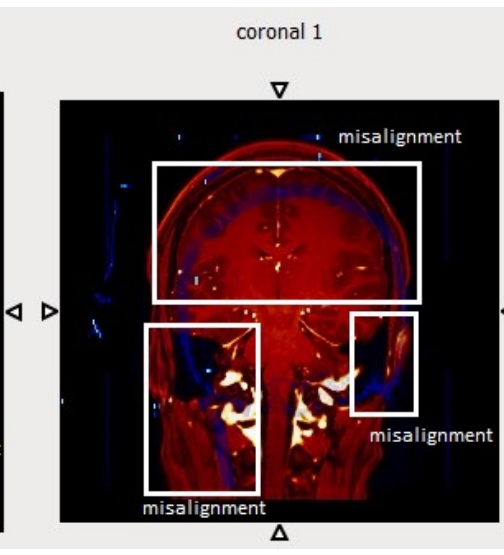

(a) Original

coronal 1

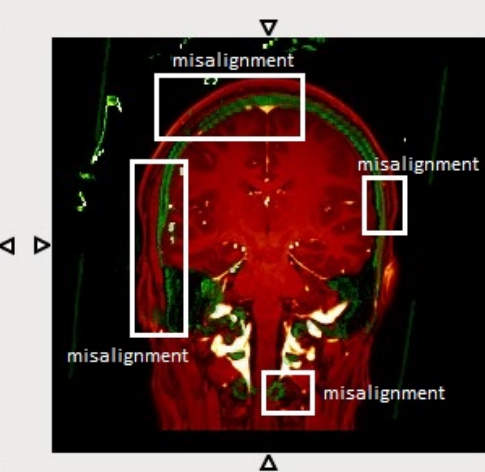

(b) SPM

coronal 1

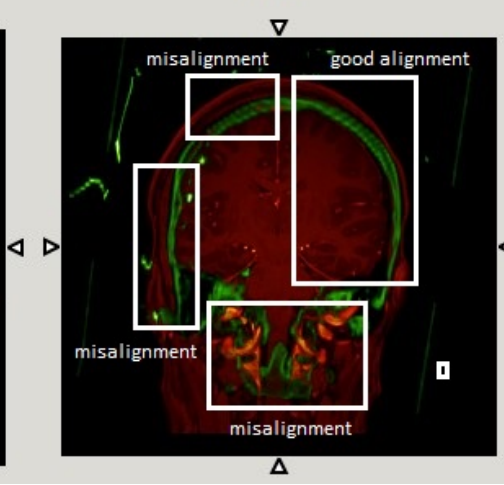

(c) FSL sagittal 1

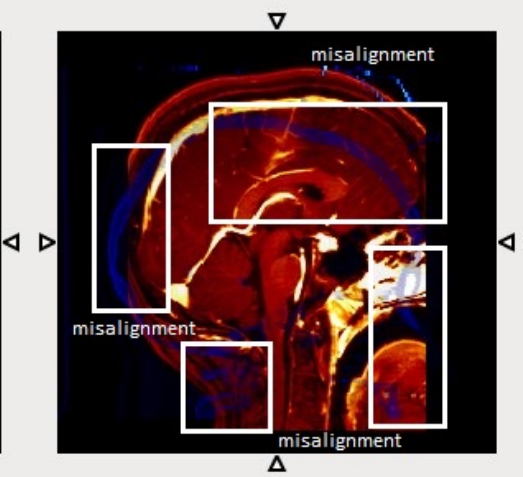

sagittal 1

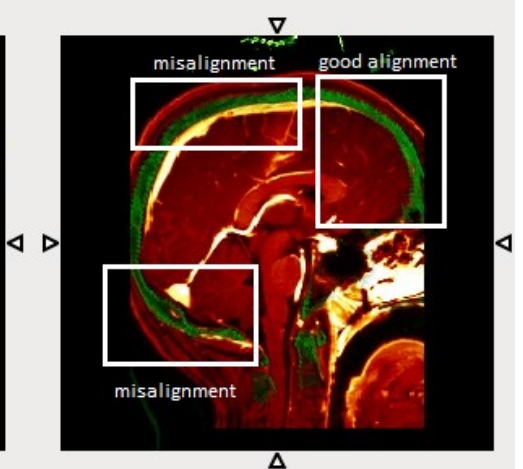

$\Delta$

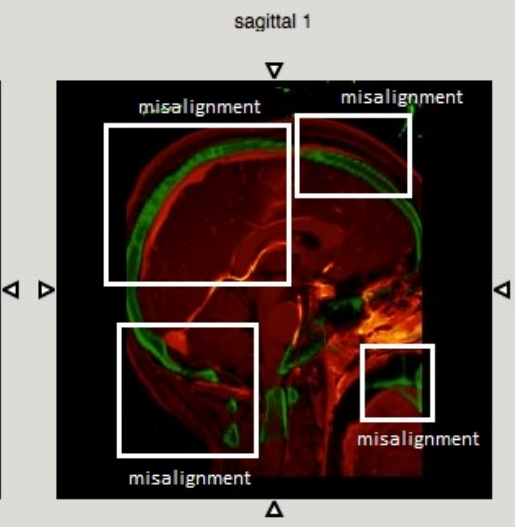



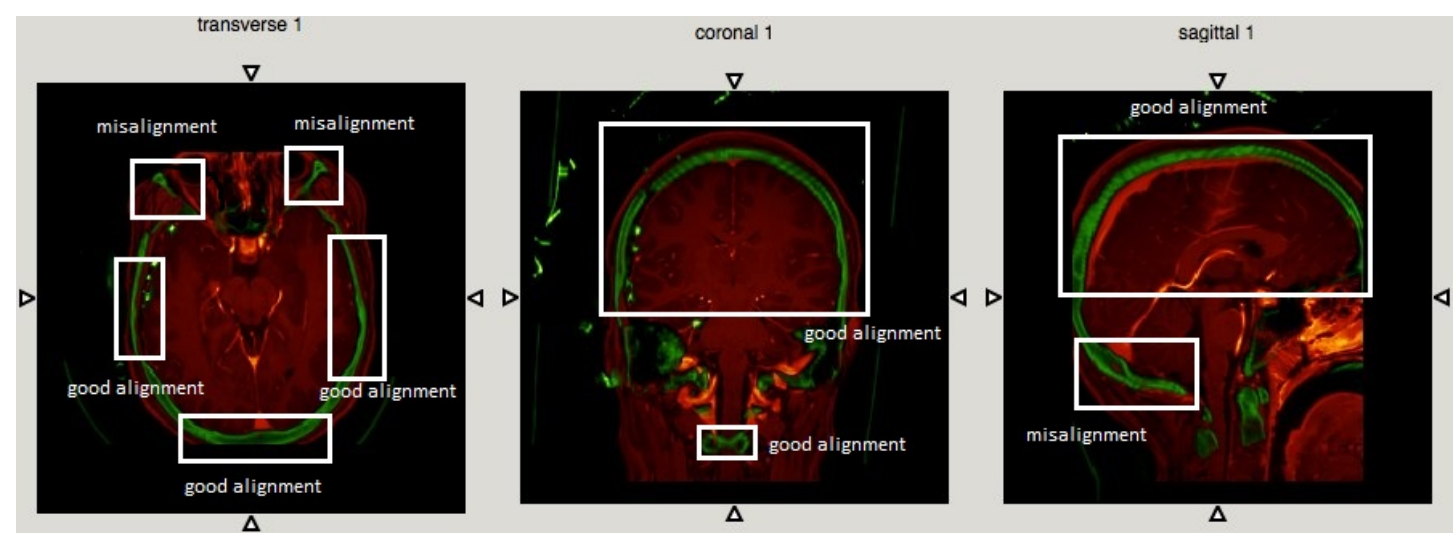

(d) The proposed method

Fig. 4.15 Patient \# 1 of data set \#2, (a) Original pre-operation MRI (appears in red) and intra-operation CT (appears in blue) are overlapped; (b) Registration results using SPM12; (c) Registration results using FSL; and (d) Registration results using the proposed method

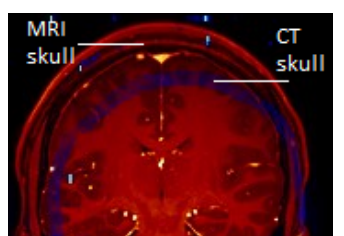

(a) Original

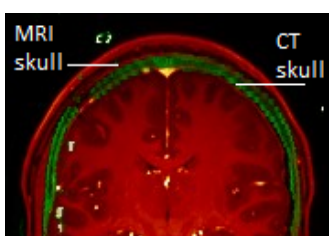

(b) SPM

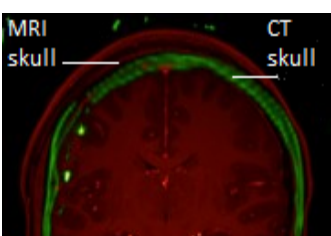

(c) FSL

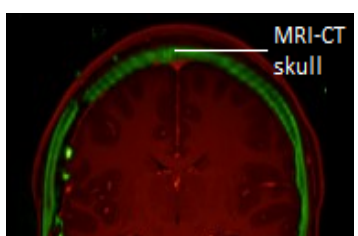

(d) Proposed method

Fig. 4.16 Enlarged skull in the coronal image

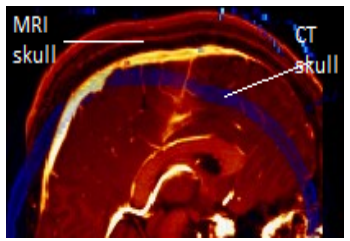

(a) Original

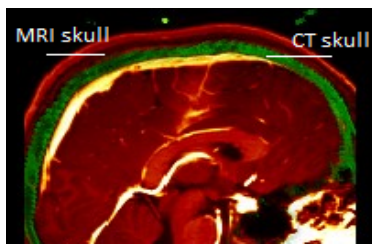

(b) SPM

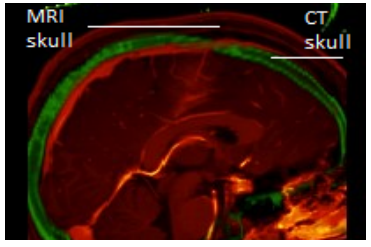

(c) FSL

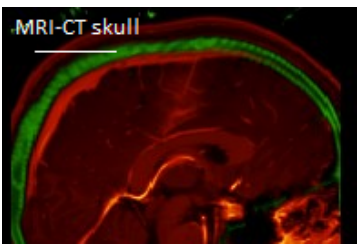

(d) Proposed method

Fig. 4.17 Enlarged skull in the sagittal image 
Table 4.5 Processing time and NMI comparison for the 5 data sets of experiment 2

\begin{tabular}{|c|c|c|c|c|}
\hline Data set \# & Evaluation parameter & SPM & FSL & The proposed method \\
\hline \multirow{2}{*}{$\# 1$} & T (s) & 112 & 303 & 181 \\
\cline { 2 - 5 } & Average NMI & 0.3516 & 0.1911 & 0.5998 \\
\hline \multirow{2}{*}{$\# 2$} & T (s) & 115 & 301 & 192 \\
\cline { 2 - 5 } & Average NMI & 0.3909 & 0.2021 & 0.6021 \\
\hline \multirow{2}{*}{$\# 3$} & T (s) & 96 & 180 & 187 \\
\cline { 2 - 5 } & Average NMI & 0.3228 & 0.2004 & 0.5717 \\
\hline \multirow{2}{*}{$\# 4$} & T (s) & 108 & 120 & 179 \\
\cline { 2 - 5 } & Average NMI & 0.3217 & 0.1909 & 0.5843 \\
\hline \multirow{2}{*}{$\# 5$} & T (s) & 105 & 300 & 181 \\
\cline { 2 - 5 } & Average NMI & 0.3071 & 0.1801 & 0.5621 \\
\hline
\end{tabular}

In Table 4.5, $\mathrm{T}$ is the processing time of the registration process, and the average NMI is the average value of the normalized mutual information between each of the registered images and the original reference image. Although the processing time is larger than SPM and less than FSL, the proposed method obtained the highest average NMI value.

\subsubsection{Registration Results of Experiment 3-MRI and PET}

According to Fig. 4.18, the registration results of the MRI and PET image, the misalignment parts are shown in the rectangle in the image (a). SPM is not working in registering those kinds of image. Image (b) is the registration results of FSL. It registers perfectly the MRI and PET in transverse, coronal, and sagittal image. Image (c) is the registration results of our method. It works in transverse image, but it miss-matching the coronal and sagittal images. Therefore, FSL is the best when register MRI and PET image compare to SPM and the proposed method. 

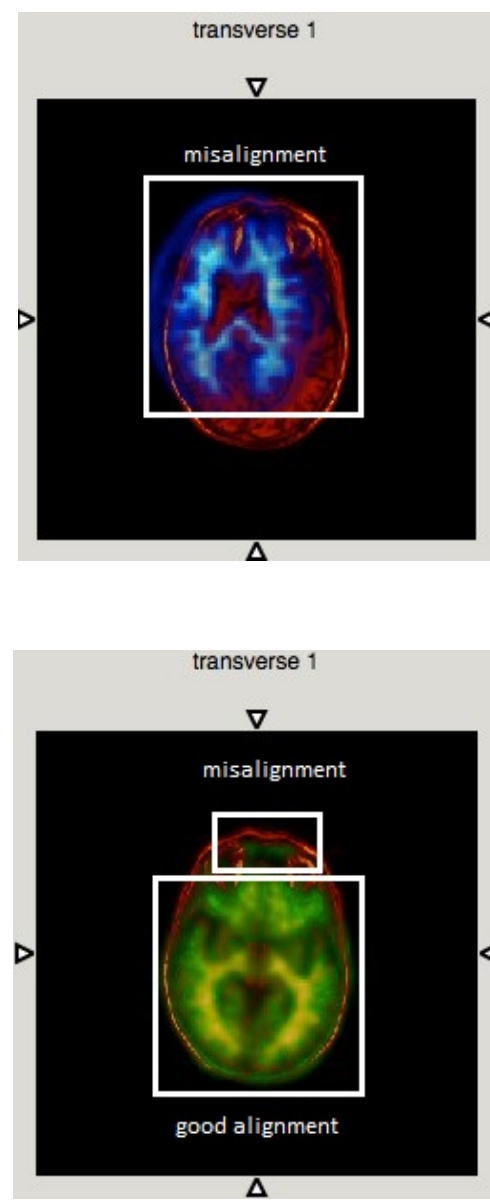

$\Delta$
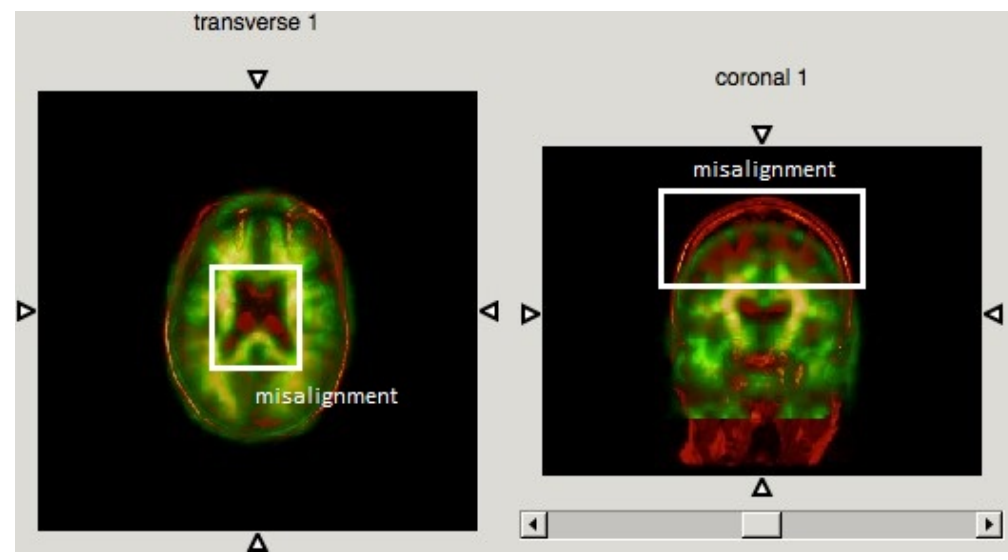

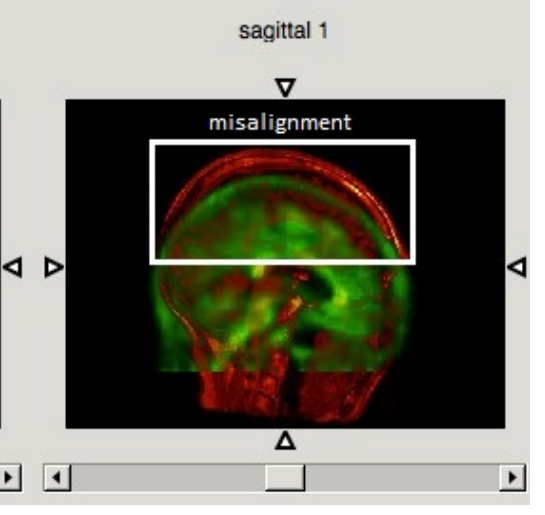

(c) The proposed method

Fig. 4.18 Patient \# 1 of data set \# 3, (a) Original MRI (appears in red) and PET (appears in blue) are overlapped; (b) Registration results using FSL ; (c) Registration results using the proposed method. 
Table 4.6 The Processing time and NMI comparison for the 5 data sets of experiment 3

\begin{tabular}{|c|c|c|c|}
\hline Data set \# & Evaluation parameter & FSL & The proposed method \\
\hline \multirow{2}{*}{$\# 1$} & T (sec) & 22 & 98 \\
\cline { 2 - 4 } & Average NMI & 0.5231 & 0.1919 \\
\hline \multirow{2}{*}{$\# 2$} & T (sec) & 22 & 87 \\
\cline { 2 - 4 } & Average NMI & 0.5621 & 0.2032 \\
\hline \multirow{2}{*}{$\# 4$} & T (sec) & 23 & 89 \\
\cline { 2 - 4 } & Average NMI & 0.5523 & 0.2040 \\
\cline { 2 - 4 } & T (sec) & 20 & 71 \\
\cline { 2 - 4 }$\# 5$ & Average NMI & 0.5237 & 0.2021 \\
\cline { 2 - 4 } & T (sec) & 20 & 69 \\
\hline
\end{tabular}

According to Table 4.6, FSL is more suitable for registering MRI and PET images than our method. FSL uses less Processing time and obtains higher average NMI value.

\subsubsection{Registration Results of Experiment 4}

According to Fig. 4.19 to 4.21 , the registration results of MRI weighted image, SPM, FSL and the proposed method all perform well here. T2, FLAIR, and GE images have been all matched well to the T1 image by those three methods. Image (b), (c), and (d) are the registration results by SPM, FSL, and our method, respectively. According to Table 4.7, $\mathrm{T}$ is the processing time of registration processing, and the average NMI is the average value of normalized mutual information between each of the registered images and the original reference image. Those three methods gained similar average NMIs. However, the processing time of our method is larger than SPM and less than FSL. 


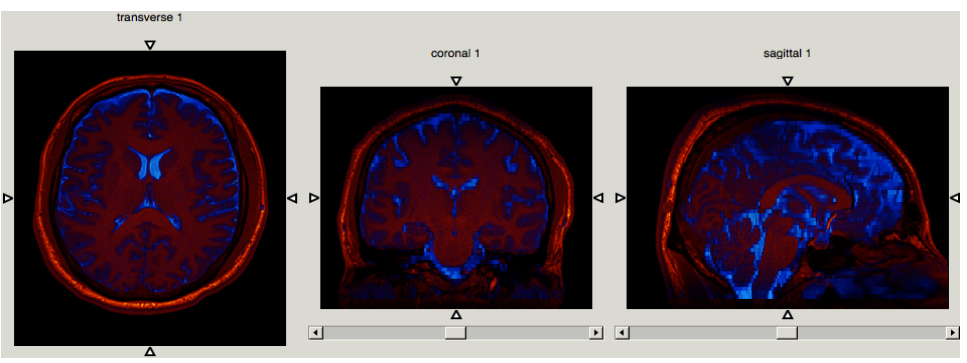

(a) Original

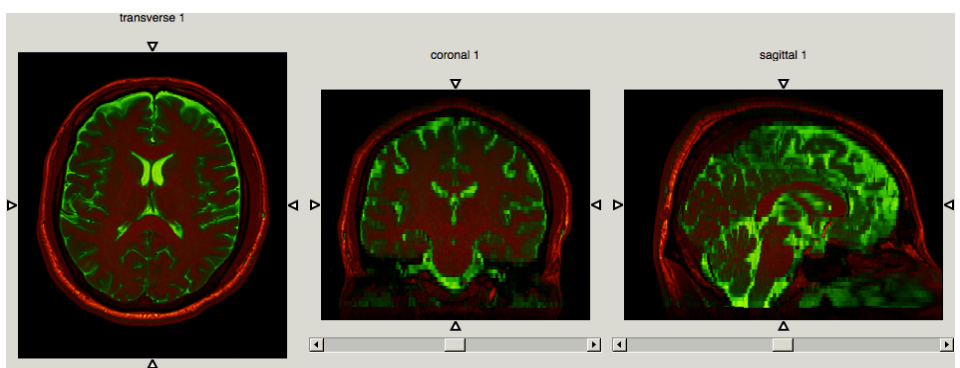

(b) SPM

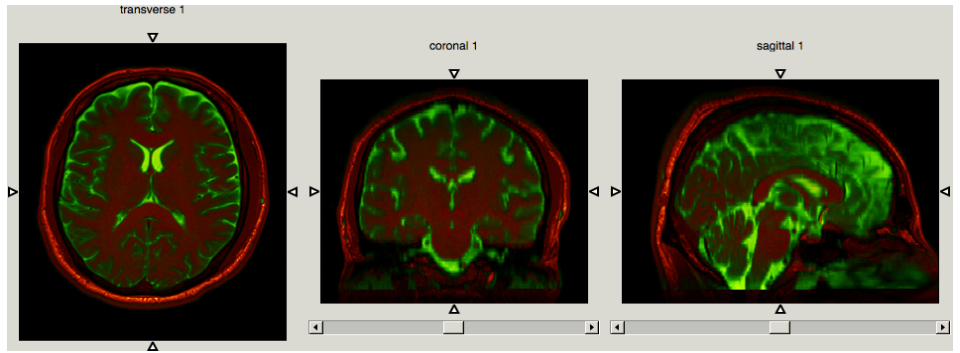

(c) FSL

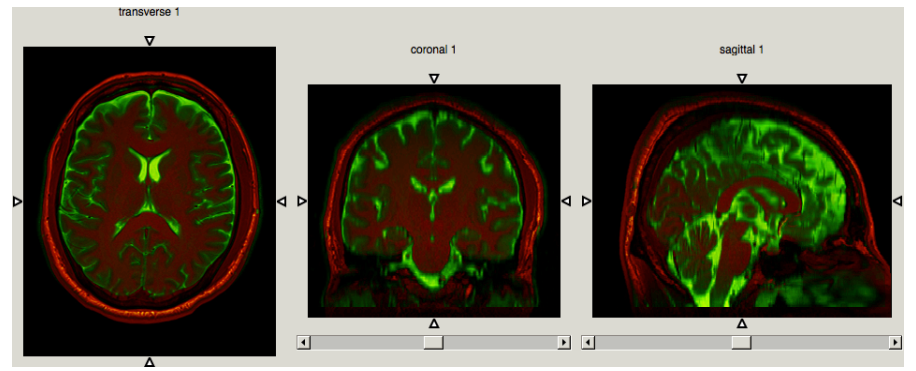

(d) The proposed method

Fig. 4.19 Registration of $\mathrm{T} 1$ and $\mathrm{T} 2$ (a) the original $\mathrm{T} 1$ (appears in red) and T2 (appears in blue) are overlapped; (b) Registration results using SPM12; (c) Registration results using FSL; and (d) Registration results using the proposed method. 


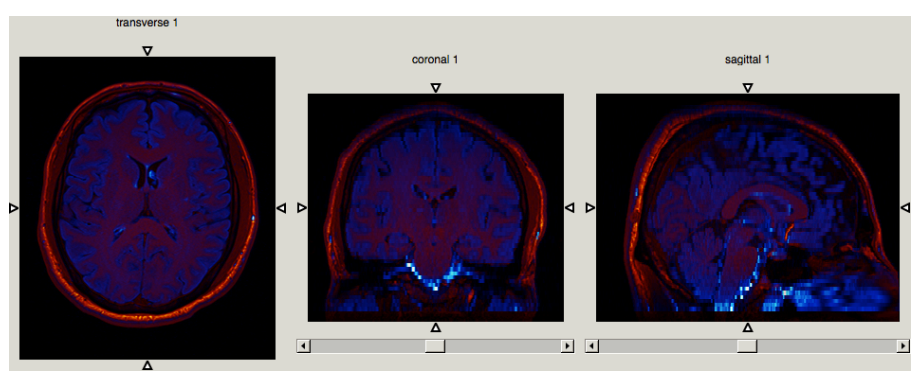

(a) Original

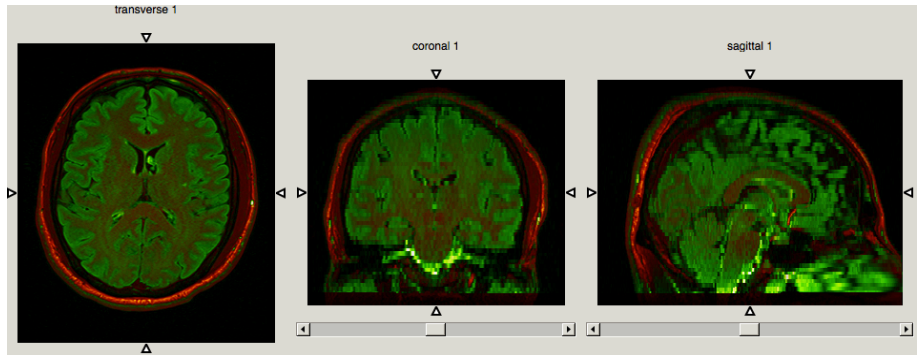

(b) SPM

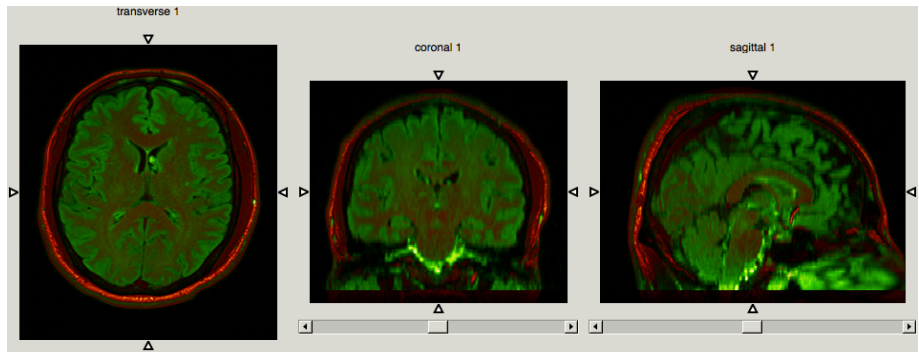

(c) FSL

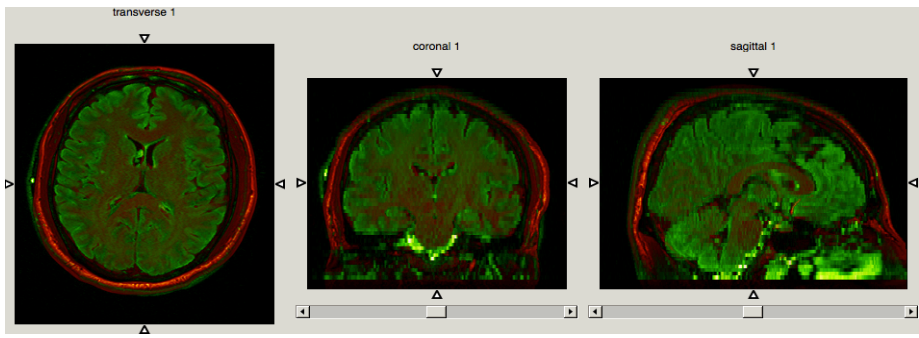

(d) The proposed method

Fig. 4.20 Registration of T1 and FLAIR (a) the original T1 (appears in red) and FLAIR (appears in blue) are overlapped; (b) Registration results using SPM12; (c) Registration results using FSL; and (d) Registration results using the proposed method. 


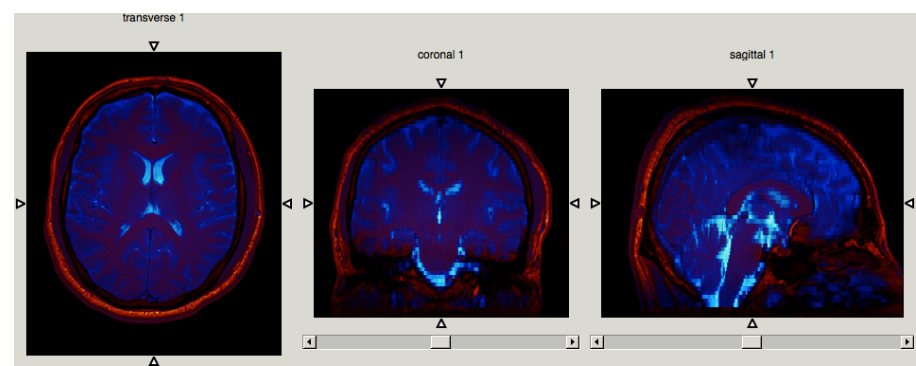

(a) Original

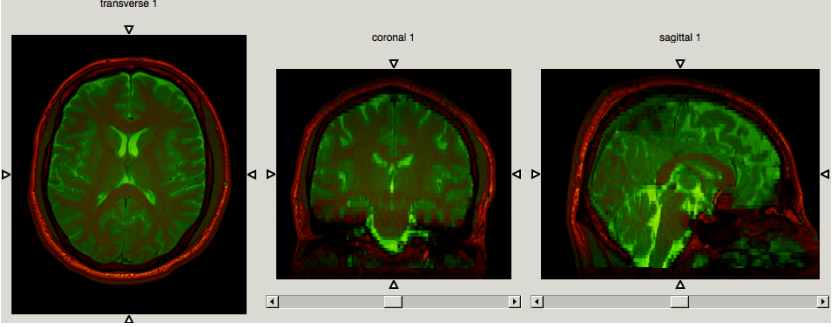

(b) SPM

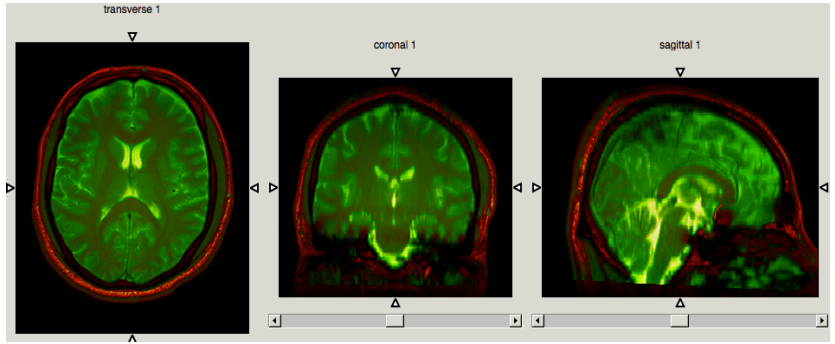

(c) FSL

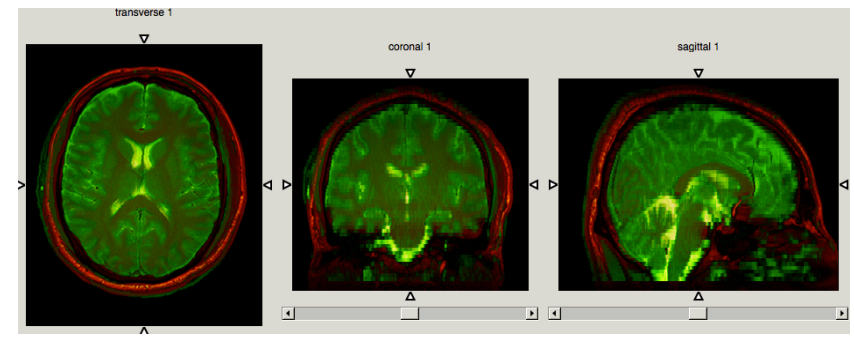

(d) The proposed method

Fig. 4.21 Registration of T1 and GE (a) the original T1 (appears in red) and GE (appears in blue) are overlapped; (b) Registration results using SPM12; (c) Registration results using FSL; and (d) Registration results using the proposed method. 
Table 4.7 Processing time and NMI comparison for the 5 data sets of experiment 4

\begin{tabular}{|c|c|c|c|c|}
\hline Data set & Evaluation parameter & SPM & FSL & The proposed method \\
\hline \multirow{2}{*}{ T2 } & $\mathrm{T}(\mathrm{s})$ & 32 & 232 & 92 \\
\cline { 2 - 5 } & Average NMI & 0.5121 & 0.5222 & 0.5223 \\
\hline \multirow{2}{*}{ FLAIR } & $\mathrm{T}(\mathrm{s})$ & 27 & 418 & 93 \\
\cline { 2 - 5 } & Average NMI & 0.5475 & 0.5374 & 0.5611 \\
\hline \multirow{2}{*}{$\mathrm{GE}$} & $\mathrm{T}(\mathrm{s})$ & 31 & 106 & 92 \\
\cline { 2 - 5 } & Average NMI & 0.4577 & 0.4522 & 0.4851 \\
\hline
\end{tabular}

\subsection{Discussion}

The proposed method has been verified to perform well when registering the whole body CT image, MRI and CT image, and MRI weighted image. However, when it comes to registering MRI and PET images, the proposed method shows good results in transverse image only. The coronal and sagittal images both have been miss-matched. The reason that led to the misalignment might be that the proposed algorithm registered the slices of the MRI and PET image along the z-axis only.

\subsection{Conclusion}

The proposed method performs well when it has been applied to inter-modality image registration, such as CT and MRI. Furthermore, the proposed method works better than SPM and FSL when applied to the whole-body CT images, and the pre-operation MRI and the intra-operation CT. For MRI and PET image registration, FSL performed best, although our method did well in the transversal direction. Our method is comparable to both FSL and SPM when registering MRI T1 to T2 and T1 to FLAIR and T1 to GE. 
The study sought and succeeded in developing an integrated registration method that is effective for registering both brain and whole-body images. The proposed method, which has integrated seamlessly a genetic algorithm for an initial optimized search, the Powell method for a secondary optimized search, Wavelet theory decomposition to minimize the processing requirements, and tri-linear interpolation and finite transformations for enhanced registration were successfully implemented to combine in one setting the excellent registration results that FMRIB Software Library (FSL) produces with brain images and the excellent results of Statistical Parametric Mapping (SPM).

The genetic algorithm was shown to be successful for determining for each image pair the three sets of registration parameters for the best matching pairs that have been obtained, defining the best solution found by the GA process. This best solution is then fed into the Powell method for a secondary search to ensure that the cost function is optimized and that the solution provided by the GA is indeed the solution for the entire 3D volume. In other words, the initial point could be the best solution, but PM will search close to this point to verify if it is the best or the PM will find the best of the best in the local area, with the best solution being the one with the highest NMI value. Therefore, PM will calculate the NMI for each solution along the assigned directions to find the maximum NMI value.

Given these determined registration parameters for all remaining slices, as defined through the optimal solution, the moving image was hence aligned to the reference image using the appropriate affine transformations through the required rotations, translations, 
and scaling as defined by the obtained registration parameters. The overlapping of the registered image and the reference image were then displayed to show the different performances of the 3 methods, namely the proposed method, SPM, and FSL by gauging the average NMI values obtained in the registration results. Visual observations are also provided in support of these NMI values. 


\section{CONCLUSIONS AND FUTURE WORK}

In this chapter, the main results of this dissertation are summarized. Then, the possible directions for future work are also discussed.

\subsection{Summary}

In this dissertation, we presented three algorithms for medical image registration. Chapter 2 to 4 describes them in detail.

In chapter 2, a semi-automatic registration method improved the CT registration of the FDG_CT and FLT_CT imaging modalities by combining the strengths of both tracers in diagnosing cancer. The registration provides a comprehensive 3D whole body combined FDG-FLT CT image. To avoid arbitrary using one set of affine transformation parameters to register images, three (the first, the middle and the last) slices were employed to calculate the whole set of transformation parameters of the 3D whole-body CT image by linear interpolation. Experimental results clearly show that the proposed method enhanced the normalized mutual information (NMI) for all the 5 sets of data and the rectangle. The NMI of set 1 is increased from 0.2065 to 0.5472 . The NMI of set 2 is increased from 0.2140 to 0.4576 . The NMI of set 3 is increased from 0.2016 to 0.5369 . The NMI of set 4 is increased from 0.2061 to 0.4776 . The NMI of set 5 is increased from 0.2326 to 0.4776 . The NMI of the rectangle is increased from 0.0029 to 0.9094 . The improvement, especially in the skull and the bottom parts of the whole body CT, can be observed visually through comparing the registered image to the original image. 
In chapter 3, The GPW method firstly selects one slice from FDG_CT and the correspondence slice from FLT_CT images. After image pre-processing process, wavelet decomposition method is to reduce the dimension of the selected two slices to get the low-frequency part of them. The Genetic algorithm applies to them and to find the best initial point for the Powell method. After the second search by the Powell method applied to the original large reference and moving images, the registration parameters will be found. Finally, the affine transformation will move the moving image to align the reference image through the found registration parameter. The GPW method improved the registration results in showing a good overlap of the two modalities and reducing the processing requirements by wavelet decomposition method. From the fusion image, the location of the cancerous lesions on the liver could be observed directly on the FLT_CT image. This outcome could help enhance the diagnosis. In retrospect, the GPW approach is shown to reduce the processing burden of GA when searching for the best global solution and prevents the PM in locking onto a best local solution for image registration, which may not be the optimal solution.

Multi-modality medical image registration is becoming more and more important nowadays. However, making a standard for evaluating the results of multi-modality image registration is difficult. In chapter 4, the primary objective is to develop an efficient method for registering the whole body FDG_CT with the FLT_CT images, the pre-operation MRI with the intra-operation $\mathrm{CT}$ images, the brain only MRI and PET images, and the MRI T1 with T2, T1 with FLAIR, T1 with GE images. A comparison of 
the results obtained will then be compared to the existing registration tools, such as Statistical Parametric Mapping (SPM) and FMRIB Software Library (FSL).

Firstly, the proposed method interpolated the original reference and moving image to unify their dimension. Three slices were chose from the reference image. In order to find the best matching for the three slices, the normalized mutual information (NMI) was calculated between each of them and every slice of the moving image. Three pairs with the highest three NMI values were chosen and applied the wavelet decomposition method to obtain the low-frequency part of the selected three pairs of images. Initial searching applied the Genetic algorithm (GA) to the three pairs of low-frequency part images to obtain three sets of registration parameters. Using them as the starting points for the Powell method (PM), and the proposed method applied the PM to the original reference and moving images to get the three sets of registration parameters again. Then, linear interpolation method was employed for them to obtain the registration parameters for all slices. Finally, the moving image was moved to align the reference image through the affine transformation. The overlapping of the registered image and the reference image were displayed to show the different performances of the three methods. The average NMI values were shown to evaluate the registration results as well. For registering the whole-body CT images registration, according to the comparison of the results of the three methods, SPM and FSL corrected more or less the misalignments in the original image. However, in the same part of the image, the proposed method registered better than them, especially for the skull and spinal column. Although the processing time was larger than SPM and less than FSL, the proposed method gained the highest average NMI 
value. For the pre-operation MRI and intra-operation CT image registration, SPM and FSL didn't register them well. Although the processing time was larger than SPM and less than FSL, the proposed method gained the highest NMI value and did the best. For MRI and PET images, FSL was the best, and the proposed method did well in the transversal image. For registering MRI T1 with T2, T1 with FLAIR, T1 with GE, the three methods gained similar average NMI values. However, the proposed method used less processing time than FSL. The proposed method is better than SPM or FSL when registering the whole-body $\mathrm{CT}$ images, and the pre-operation MRI with the intraoperation CT images. For MRI and PET image registration, FSL performed best, although our method did well in the transversal direction. Our method is comparable to both FSL and SPM when registering MRI T1 with T2, and T1 with FLAIR, and T1 with GE. To improve the proposed method for MRI and PET images, registering them along the y-axis, and z-axis could perhaps improve the results but at an additional cost of processing time.

This research endeavor has thus shown that when registration of multiple modalities is performed optimally, it allows for

- Developing imaging algorithms, in a common 3D space, that allows for contextual analysis, exploiting simultaneously both anatomic structure and functional or metabolic correlates and dynamics, in both normal states and under specific pathologic conditions. 
- Far-improved mapping of regions of interest, leading to enhanced diagnosis, better treatment planning, and safer surgical outcomes.

- Multimodal imaging with the ability to consolidate singular capabilities each modality brings, and to create a cohesive platform that could combine structure to functional correlates and/or consolidate high spatial resolution with high temporal resolution.

- Meet the challenges of database design and management that are augmented with mechanisms for visualization on a common 3D space with fast user interaction and, as a consequence, effective methods of data representation, visualization, and mining could be performed.

- Resolve compatibility problems arising from the use of dissimilar recording modalities and diverse software platforms.

We will be looking in the future as to why only the transversal direction gave us good alignment when registering MRI and PET, this was an intriguing outcome. To improve our algorithm, registering images along the y-axis, and z-axis could perhaps improve these results but at an additional of the cost of processing time. 


\subsection{Future Work}

In today's healthcare delivery, imaging plays an important role throughout the entire clinical process, from diagnostics and treatment planning to surgical procedures and follow-up. Since most imaging modalities are digital, with continually increasing resolution, medical image processing has to face the challenges coming from fusing multiple images recorded from different modalities. Another issue that is also challenging is the processing time required for dealing with large datasets. As a consequence, a significant amount of research is focused on advanced parallelization techniques in order to achieve acceptable real-time response [67]. Also increasing use of dynamic acquisition for example perfusion MRI will have need of using registration mechanisms that could overcome for patients' motion correction [68]. Moreover, during both the intra-operation imaging and non-interventional imaging, many body organs, such as heart and lungs have natural elastic motion. More complex, in image-guided surgery, the intra-operation images are deformed with respect to the pre-operation image, due to tissue shift and breathing motion.

Image registration could be applied to all of those situations to correct for body motion. Although rigid body registration is still dominant in the literature, the non-rigid body registration is increasingly applied. The non-rigid and elastic method might lead to solutions that are correct from a geometrical point, but they are not anatomically meaningful. Further study on realistic deformation methods reflecting tissue properties would improve the accuracy of the estimation and attain acceptance from the physicians [69]. 


\section{LIST OF REFERENCES}

[1] J. G. Betts, P. DeSaix, E. Johson, and J. E. Johnson, Anatomy \& Physiology. Houston, TX: OpenStax College, pp. 36-37, 2013.

[2] J. B. A. Maintz, and M. A. Viergever, "A survey of medical image registration", Medical Image Analysis, vol. 2, issue 1, pp. 1-36, 1998.

[3] M. A. Viergevera, J. B. A. Maintzb, S. Kleinc, K. Murphyd, M. Staringe, and J. P.W. Pluimf, "A survey of medical image registration-under review", Medical Image Analysis, vol. 33, pp. 140-144, 2016.

[4] J. P.W. Pluimf, J. B. A. Maintz, and M. A. Viergevera, "Mutual-information-based registration of medical images: A survey", IEEE transactions on medical imaging, vol. 22, issue 8, pp. 986-1004, 2003.

[5] T. M. Lehmann, C. Go nnner, and K. Spitzer, "Survey: Interpolation methods in medical image processing", IEEE transactions on medical imaging, vol. 18, issue 11, pp. 1049-1075, 1999.

[6] L. G. Brown, "A survey of image registration techniques", ACM Computer Surveys, vol. 24, pp. 325 - 375, 1992.

[7] J. B. Maintz, "Retrospective registration of tomographic brain images", Ph.D. Thesis, Netherland: University of Utrecht.

[8] P. A. van den Elsen, E. J. D. Pol, "Medical image matching: a review with classification”, IEEE Engineering in Medicine and Biology, pp. 26- 39, 1993.

[9] P. A. Freeborough, N. C. Fox, "Measurement of global and regional cerebral volume changes by integrating boundary shifts between registered serial 3D MR scans", Information Processing in Medical Imaging, Berlin: Springer, pp. 355368, 1997.

[10] J. V. Hajnal, N. Saeed, A. Oatridge, E. J. Williams, I. R. Young, G. M. Bydder, "Detection of subtle brain changes using sub-voxel registration and subtraction of 
serial MR images", Journal of Computer Assisted Tomography, vol. 19, pp. 677 691, 1995.

[11] D. J. Hawkes, "Algorithm for radiological image registration and their clinical application", Journal of Anatomy, pt. 3. 1993.

[12] D. L. Collins, T. M. Peters, A. C. Evans, "An automated 3D nonlinear image deformation procedure for determination of gross morphometric variability in human brain”, Visualization in Biomedical Computing, SPIE, vol. 2359, pp. 180190, 1994.

[13] F. L. Bookstein, "Thin-plate splines and the atlas problem for biomedical images", Information Processing in Medical Imaging, Heidelberg: Springs, pp. 326-342, 1991.

[14] T. F. Cootes, A. Hill, C. J. Taylor, J. Haslam, "The use of active shape models for locating structures in medical images", Image and Vision Computing, vol. 12, pp. 276-285, 1994.

[15] J. A. Parker, R. V. Kenyon, and D. E. Troxel, "Comparison of interpolating methods for image resampling ", IEEE Trans. Med. Imag., vol. MI-2, pp. 31-39, 1983.

[16] X. Wang, J. Wu, J. Wang, and M. Adjouadi, "Super-resolution analysis for passive microwave images using FIPOCS", Computer Vision, Image Analysis and Processing, vol. 87831L, pp. 1-5, 2013.

[17] F. Maes, A. Collignon, D. Vandermeulen, G. Marchal, and P. Suetens, "Multimodality image registration by maximization of mutual information", IEEE Transactions on Medical Imaging, vol. 16, issue 2, pp.187-198, 1997.

[18] A. Gholipour, N. Kehtarnavaz, R. Briggs, M. Devous, and K. Gopinath, "Brain functional localization: A survey of image registration techniques", IEEE transactions on medical imaging, vol. 26, issue 4, pp. 427-451, 2007.

[19] S. Damas, O. Cordón, and J. Santamaría, "Medical Image Registration Using Evolutionary Computation: An Experimental Survey, IEEE Computational Intelligence Magazine, vol. 6, issue 4, pp. 26-42, 2011. 
[20] M. Khader, and A. B. Hamza, "An information-theoretic method for multimodality medical image registration", Expert System with Applications, vol. 39, issue 5, pp. 5548-5556, 2012.

[21] O. K. Erol, and I. Eksin, "A new optimization method: Big Bang- Big Crunch", Advances in Engineering Software, vol. 37, pp. 106-111, 2006.

[22] X. Wang, and M. Adjouadi, "Automatic registration of FDG_CT and FLT_CT images integrating Genetic Algorithm, Powell method and wavelet decomposition", IEEE Conf. Signal Processing in Medicine and Biology Symposium, pp. 1-5, 2015.

[23] J. M. Rouet, J. J. Jacq, and C. Roux, "Genetic algorithms for a robust 3-D MR-CT registration", IEEE Trans. Info. Tec. In Biomedicine, vol. 4, pp. 126-136, 2002.

[24] A. Hill, C.J. Taylor, "Model-based image interpretation using genetic algorithms", Image and Vision Computing, vol. 10, pp. 295-300, 1992.

[25] H. Y. Zhang, X. Z. Zhou, J. Z. Sun, and J. W. Zhang, "A novel medical image registration method based on mutual information and genetic algorithm ", Computer Graphics, Imaging and Vision: New Trends, 2005.

[26] M. Bhattacharya and A. Das, "Multi resolution medical image registration using maximization of mutual information and optimization by genetic algorithm", Nuclear Science Symposium Conference Record, 2007.

[27] V. T. Ingole, C. N. Deshmukh, A. Joshi, and D. Shete, "Medical image registration using genetic algorithm", Emerging Trends in Engineering and Technology, 2009.

[28] M. Jenkinson, and S. Smith, "A global optimization method for robust affine registration of brain images", Medical Image Analysis, vol. 5, issue 2, pp. 143-156, 2001.

[29] M. Jenkinson, P.R. Bannister, J.M. Brady, and S.M. Smith, "Improved optimisation for the robust and accurate linear registration and motion correction of brain images", NeuroImage, 17(2): 825-841, 2002. 
[30] W. Chen, Z. Mo, and W. Guo, "Detection of QRS complexes using wavelet transformation and golden section search algorithm", PubMed, 26(4): 748-51, 2009.

[31] X. M. Cao, Q. Q. Ruan, "A Survey on Evaluation Methods for Medical Image Registration", IEEE/ICME International Conference on Complex Medical Engineering, vol.1-4, pp.718-721, 2007.

[32] F. Alam, S. U. Rahman, S. Khusro, S. Ullah, and A. Khalil,"Evaluation of Medical Image Registration Techniques Based on Nature and Domain of the Transformation", Journal of Medical Imaging and Radiation Sciences, vol. 47, issue 2, pp. 178-193, 2016.

[33] Members \& collaborators of the Wellcome Trust Centre for Neuroimaging, SPM, 2016, [online] available: http://www.fil.ion.ucl.ac.uk/spm/.

[34] The Analysis Group, FMRIB, Oxford, UK. 2015, [online] available: http://fsl.fmrib.ox.ac.uk/fsl/fslwiki/.

[35] C. C. Gaudes, D. V. Ville, F. Grouiller, R. Thornton, L. Lemieux, M. Seeck, F. Lazeyras, S. Vulliemoz, "Mapping interictal epileptic discharges using mutual information between concurrent EEG and fMRI", NeuroImage, vol. 68, pp. 248$262,2013$.

[36] A.Wolbarst, W.Hendee, "Evolving and experimental technologies in medical imaging," Radiology, vol. 238, pp. 16-39, January 2006.

[37] J. Maintz, M. Viergever, "A survey of medical image registration," Medical Image Analysis, vol. 2, pp. 1-36, 1998.

[38] A. Ardeshir Goshtasby, "2-D and 3-D Image Registration for Medical", Remote Sensing, and Industrial Applications, Wiley Press, 2005.

[39] F. Candocia, M. Adjouadi, "A similarity measure for stereo feature matching", Image Processing, IEEE Trans., vol. 6, pp. 1460-1464, 1997.

[40] M. Adjouadi, F. Candocia, J. Riley, “Exploiting Walsh-based attributes to stereo vision”, Signal Processing, IEEE Trans, vol. 44, pp.409-420, 1996 
[41] A.Rangarajan, J. Duncan, "Matching point features using mutual information", Biomedical Image Analysis, pp. 172-181, 1998.

[42] V. Potesil, T. Kadir, S.M.Brady, "Learning New Parts for Landmark Localization in Whole-Body CT Scans", Medical Imaging, IEEE Trans.,vol. 33, pp.836-848, 2014.

[43] M. Wang, J. Wu, etc., "A landmark based registration technique for minimally invasive spinal surgery”, Consumer Electronics, pp. 235-236, 2013.

[44] A.Varnavas, T.Carrell, G.Penney, "Increasing the Automation of a 2D-3D Registration System”, Medical Imaging, IEEE Trans, vol. 32, pp. 387- 399, 2013.

[45] Z. Song, S. Zhou, J. Guan, "A Novel Image Registration Algorithm for Remote Sensing Under Affine Transformation", Geoscience and Remote Sensing, IEEE Trans., vol. 52, pp. 4895-4912, 2014.

[46] A. Wolbarst, W. Hendee, "Evolving and experimental technologies in medical imaging," Radiology, vol. 238, pp. 16-39, January 2006.

[47] J. Maintz, M. Viergever, "A survey of medical image registration," Medical Image Analysis, vol. 2, pp. 1-36, 1998.

[48] B. Zitova', J. Flusser, "Image registration methods: a survey", Image and Vision Computing, vol. 21, pp.977-1000, 2003.

[49] W. M. Wells, P. Viola, H. Atsumi, S. Nakajima, R. Kikinis, "Multi-modal volume registration by maximization of mutual information", Medical Image Analysis, vol. 1, pp.35-51, 1996.

[50] L. Tang, G. Hamarneh, A. Celler, "Co-registration of bone CT and SPECT images using mutual information", IEEE International Symposium on Signal Processing and Information Technology, pp. 116-121, 2006.

[51] X. Wang, Y. Liu, Y. Huang, "The application of image registration based on genetic algorithm with real data", Synthetic Aperture Radar, IEEE Conference, pp. 844-847, 2009. 
[52] X. Huang, F. Zhang, "Multimodal Medical Image Registration Based on Gradient of Mutual Information and Hybrid Genetic Algorithm", Intelligent Information Technology and Security Informatics, Third International Symposium, pp. 125$128,2010$.

[53] X. G. Du, J. W. Wu, Y. P. Wang, X. G. Liu, and S. Li, "An algorithm MultiReselotion Medical Image Registration Based on Firefly Algorithm and Powell", Intelligent System Design and Engineering Applications, IEEE Conference, pp. 274-277, 2013.

[54] Y. Lei, and Y. Zhang, "An improved 2D-3D Medical Image Registration algorithm Based on Modified Mutual Information and Expended Powell Method", Medical Imaging Physics and Engineering, IEEE International Conference, pp. 24-29, 2013.

[55] T. M. Lehman, C. Gonner, and K.Spitzer, "Interpolation Methods in Medical Image Processing", IEEE transactions on medical imaging, vol. 18, pp. 10491075, 1999.

[56] O. D. Evans and Y. Kim, "Efficient implementation of image warping on a multimedia processor," Real-Time Imaging, vol. 4, pp. 417-428, 1998.

[57] P. E. Danielsson and M. Hammerin, "Note: High accuracy rotation of images", CVGIP: Graph. Models Image Processing, vol. 54, no. 4, pp. 340-344, 1992.

[58] M. Unser, A. Aldroubi, and M. Eden, "Fast B-splines transforms for continuous image representation and interpolation", IEEE Trans. Pattern Anal. Machine Intell., vol. 13, pp. 277-285, 1991.

[59] N. A. Dodgson, "Quadratic interpolation for image resampling," IEEE Trans. Image Processing, vol. 6, pp. 1322-1326, 1997.

[60] M. Unser, P. The venaz, and L. Yaroslavsky, "Convolution based interpolation for fast, high-quality rotation of images", IEEE Trans. Image Processing, vol. 4, pp. 1371-1381, 1995.

[61] C. Lee, M. Eden, and M. Unser, "High-quality image resizing using oblique projection operators", IEEE Trans. Image Processing, vol. 7, no. 5, pp. 679-692, 1998. 
[62] W. Chen, Z. Mo, and W. Guo, "Detection of QRS complexes using wavelet transformation and golden section search algorithm", PubMed, 26(4): 748-51, 2009.

[63] E. Biagi, L. Breschi, S. Granchi, L. Masotti, G. Cincotti, G. Loi, and, M. Pappalardo, "Comments on "Frequency decomposition and compuyting of ultrasound medical images with wavelet packets"', IEEE Transaction on Medical Imaging, Vol. 21, Issue 7, pp. 834-837, 2002.

[64] S. R. Dubey, S. K. Singh, and R. K. Singh, "Local wavelet pattern: a new feature descriptor for image retrieval in medical CT databases", IEEE Transaction on Image Processing, Vol. 24, Issue 24, pp. 5892-5903, 2015.

[65] H. L. Shi, and B. Hu, "Image registration using a new scheme of wavelet decomposition", 2008 IEEE Instrumentation and Measurement Technology Conference, pp. 235-239, 2008.

[66] I. Scholl, T. Aach, T. M. Deserno, T. Kuhlen, "Challenges of medical image processing”, Compute Sci Res Dev, vol. 26, pp. 5-13, 2011.

[67] D. L. G. Hill, P. G. Batchelor, M. Holden, D. J. Hawkes, "Medical image registration", Physics in Medicine and Biology, vol. 46, R1-R45, 2000.

[68] E. Ferrante, N. Paragios, "Slice-to-volume medical image registration: a survey", Medical Image Analysis, vol. 39, pp. 101-123, 2017. 
VITA

\section{XUE WANG}

1985 Born, Wuhan, China

2007 B.S., Communication Engineering

Wuhan University

Wuhan, China

2011 M.S., Circuits and System

Wuhan University of Science and Technology

Wuhan, China

2017 Ph.D. Candidate, Electrical Engineering

Florida International University

Miami, Florida

\section{PUBLICATION AND PRESENTATIONS}

1. Xue Wang, Chunfei Li, Mohammed Z. Goryawala, and Malek Adjouadi "Multimodality medical image registration method compared with Statistical Parametric Mapping and FMRIB Software Library", under review, IEEE Trans. On Medical Imaging.

2. Xue Wang and Malek Adjouadi, "Automatic registration of FDG_CT and FLT_CT images intergrating Genetic Algorithm, Powell Method, and wavelet decomposition", IEEE Signal Processing in Medicine and Biology Symposium (SPMB 2015), pp. 1-5, 2015.

3. Xue Wang, Zhenzhong Wang, Jin Wang, Mercedes Cabrerizo, , Mohammed Z. Grayawala, Seza A. Gulec, and Malek Adjouadi "A novel semi-automatic method for accurate registration of FDG_CT and FLT_CT image modalities", IEEE Signal Processing in Medicine and Biology Symposium (SPMB 2015), pp. 1-5, 2015.

4. Xue Wang, Jin Wu, Jin Wang, and Malek Adjouadi, "Super-resolution analysis for passive microwave images using FIPOCS", Proc. SPIE 8783, Fifth International Conference on Machine Vision (ICMV 2012): Computer Vision, Image Analysis and Processing, 878305(March 13, 2013). 